\title{
Request for Modification of 200 Area Effluent Treatment Facility Final Delisting
}

Date Published

November 1998

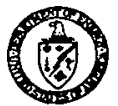

United States

Department of Energy

P.O. Box 550

Richland, Washington 99352 


\section{RELEASE AUTHORIZATION}

Document Number: $\quad$ DOE/RL-98-62, Rev. 0

$\begin{array}{ll}\text { Recument Title: } & \text { Request for Modification of } 200 \text { Area } \\ \text { Treatment Facility Final Delisting }\end{array}$

This document, reviewed in accordance with DOE Order 241.1, "Scientific and Technical Information Management,"and 241.1-1, "Guide to the Management of Scientific and Technical Information," does not contain classified or sensitive unclassified information and is:

\section{APPROVED FOR PUBLIC RELEASE}

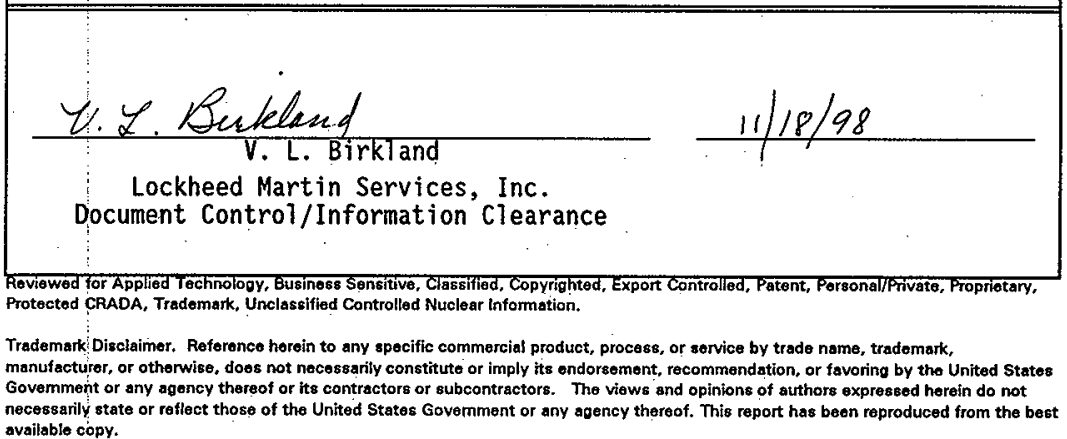

Printed in the United States of America.

Avsilable to the U.S. Department of Energy and ite contractors trom the U.S. Department of Eneroy Otfice of Scientific and Technical Information, P.O. Box 62, Oak Ridos, TN 37831: Tolephono: 423/576-8401.

Available to the public from the U.S. Department of Commerce National Technical Information Service, 5285 Port Royal Road, Springfield: VA 22161: Telephone: 703/487-4650. 


\section{SUMMARY}

4 A Delisting Petition submitted to the U.S. Environmental Protection Agency in August 1993 addressed 5 effluent to be generated at the 200 Area Effluent Treatment Facility from treating Hanford Facility waste 6 streams. This Delisting Petition requested that 71.9 million liters per year of treated effluent, bearing the 7 designation 'F001' through 'F005', and/or 'F039' that is derived from 'F001' through ' $\mathrm{F} 005$ ' waste, be 8 delisted. On June 13, 1995, the U.S. Environmental Protection Agency published the final rule (Final

9 Delisting), which formally excluded 71.9 million liters per year of 200 Area Effluent Treatment Facility

10

11 effluent from "being listed as hazardous wastes" (60 FR 31115 now promulgated in 40 CFR 261). Given the limited scope, it is necessary to request a modification of the Final Delisting to address the management of a more diverse multi-source leachate (F039) at the 200 Area Effluent Treatment Facility.

From past operations and current cleanup activities on the Hanford Facility, a considerable amount of both liquid and solid Resource Conservation and Recovery Act of 1976- regulated mixed waste has been and continues to be generated. Ultimately this waste will be treated as necessary to meet the Resource Conservation and Recovery Act Land Disposal Restrictions. The disposal of this waste will be in Resource Conservation and Recovery Act-compliant permitted lined trenches equipped with leachate collection systems. These operations will result in the generation of what is referred to as multi-source leachate. This newly generated waste will receive the listed waste designation of F039. This waste also must be managed in compliance with the provisions of the Resource Conservation and Recovery Act.

Given the nature of past operations and current waste management activities, leachate resulting from Hanford Facility mixed waste disposal operations is anticipated to represent the broadest spectrum of constituents destined for disposal of a multi-source leachate waste stream to be managed at the 200 Area Effluent Treatment Facility. The Liquid Effluent Retention Facility and the 200 Area Effluent Treatment Facility are Resource Conservation and Recovery Act-compliant permitted waste management units that will be used to manage this waste stream. Following treatment at the 200 Area Effluent Treatment Facility, the treated leachate will be discharged to the State-Approved Land Disposal Site north of the 200 West Area in compliance with the Washington State Waste Discharge Permit (ST 4500).

Because the treated leachate also will be considered a hazardous waste under the derived-from rule, and the Resource Conservation and Recovery Act regulations prohibit the placement of liquid hazardous waste on land (40 CFR 264.314), this effluent must be delisted before being discharged to the State-Approved Land Disposal Site. The effluent also must meet the discharge criteria of the State Waste Discharge Permit (ST 4500) and the existing Final Delisting (40 CFR 261, Appendix IX, Table 2).

In accordance with the requirements of Washington Administrative Code 173-303-072 (1) and (4) and $-910(3)$, and 40 CFR 260.20 and .22, this delisting modification document presents sufficient information to justify that treated multi-source leachate "is not capable of posing a substantial present or potential threat to public health or the environment. . .". This delisting modification document applies an upfront delisting approach depends on the ability of the treated effluent to meet concentration levels specified in the Final Delisting. The management of this waste from the point of generation through disposal is explained. A demonstration is made showing how the constituents will be effectively treated so that 200 Area Effluent Treatment Facility effluent will no longer require management as a regulated dangerous waste or hazardous waste. The demonstration is based on treatment knowledge gained from 200 Area Effluent Treatment Facility operations, surrogate testing performed in support of the original delisting effort, and information available from the manufacturer for equipment used in the 200 Area Effluent Treatment Facility. In 
1 addition, details are provided showing how constituents included in the treated waste stream will be

2 monitored to confirm applicable constituent concentration requirements are met.

3

4 It is believed that the waste management process described in this document represents the best

5 management system available for multi-source leachate. Given the cradle-to-grave control of multi-source

6 leachate, the treatment capabilities of the 200 Area Effluent Treatment Facility, and the regulatory control

7 over this process, the treated multi-source leachate "is not capable of posing a substantial present or

8 potential threat to public health or the environment. . "[WAC 173-303-072(4)]. 
CONTENTS

3 SUMMARY. iii

5 GLOSSARY. vii

7 METRIC CONVERSION CHART viii

ADMINISTRATIVE INFORMATION .

LEACHATE GENERATION AND MANAGEMENT

Mixed Waste Disposal Acceptance Criteria

Expected Leachate Volumes and System Capacities .................................................................2-4

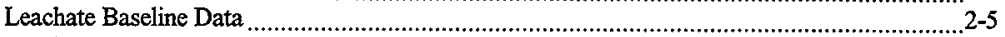

\section{AREA EFFLUENT TREATMENT FACILITY AND LIQUID EFFLUENT} RETENTION FACILITY PROCESS LIQUID EFFLUENT RETENTION FACILITY PROCESS DESCRIPTION

Primary Treatment Train

Secondary Treatment Train

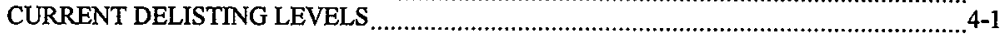

STANDARDS TREATED WASTE MUST MEET …….............................................4-4 


\section{FIGURES}

Plan View of the 200 Area Effluent Treatment Facility...................................... $3-9$

18 Figure 3-3. 200 Area Effluent Treatment Facility Layout. ..........................................................3-10

19 Figure 3-4. 200 Area Effluent Treatment Facility.

Reverse Osmosis Unit. ................................................ 3-16

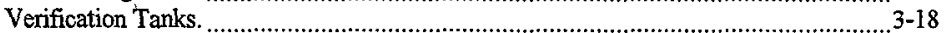

Effluent Treatment Facility Evaporator. .............................................................. $3-19$

27 Figure 3-12.

28 Figure 3-13!

Thin Film Dryer.

\section{TABLES}

35 Table 2-2. Baseline Constituents Above Method Detection Limits. ............................................. 2-12

36 Table 2-3. Identification of Potential Leachate Constituents.

37 Table 4-1. Constituents Delisted and Considered in Delisting Modification...........................................4-7

38 Table 4-2. Treatability Groups.

39 Table 4-3. Organic Constituent Modified Treatability Envelope. ..........................................................4-19

40 Table 4-4. Standards Treated Waste Must Meet. ............................................................................4-28 


\section{GLOSSARY}

ARAR

ASTM

6

BDAT

8

9 CAS

10 CERCLA

11 CFR

12

13 DOE-RL

14

15 Ecology

$16 \mathrm{EE} / \mathrm{O}$

17 EPA

18 ERDF

19 ETF

20

21 FR

22

$23 \mathrm{HBL}$

24 HDPE

25

26 ICP

27 IX

28

29 LDR

30 LERF

31 LLBG

32

33 PC

34 PCB

35 PUREX

36

37 RCRA

$38 \mathrm{RO}$

39

40 TSCA

41

42 UV/OX

43

44 WAC

45

$46{ }^{\circ} \mathrm{C}$

47

$48 \mathrm{pCi} / \mathrm{L}$

$49 \mathrm{pH}$

$50 \mathrm{ppm}$

51

applicable or relevant and appropriate requirements

American Society for Testing and Materials

best demonstrated available technology

Chemical Abstract Service

Comprehensive Environmental Response, Compensation, and Liability Act of 1980

Code of Federal Regulations

U.S. Department of Energy, Richland Operations Office

Washington State Department of Ecology

Electrical Energy per Order

U.S. Environmental Protection Agency

Environmental Restoration Disposal Facility

200 Area Effluent Treatment Facility

Federal Register

health-based level

high-density polyethylene

inductively coupled plasma

ion exchange

land disposal restriction

Liquid Effluent Retention Facility

Low-Level Burial Grounds

process condensate

polychlorinated biphenyl

plutonium-uranium extraction (Facility)

Resource Conservation and Recovery Act of 1976

reverse osmosis

Toxic Substances Control Act of 1976

ultraviolet oxidation

Washington Administrative Code

degrees Celsius

picocuries per liter

negative logarithm of the hydrogen-ion concentration

parts per million 
Into metric units

Out of metric units

\begin{tabular}{|c|c|c|c|c|c|}
\hline If you know & Multiply by & To get & If you know & Multiply by & To get \\
\hline \multicolumn{3}{|c|}{ Length } & \multicolumn{3}{|c|}{ Length } \\
\hline inches : & 25.40 & Millimeters & millimeters & 0.0393 & inches \\
\hline inches & 2.54 & Centimeters & centimeters & 0.393 & inches \\
\hline feet & 0.3048 & Meters & meters & 3.2808 & feet \\
\hline yards & 0.914 & Meters & meters & 1.09 & yards \\
\hline miles & 1.609 & Kilometers & kilometers & 0.62 & miles \\
\hline \multicolumn{3}{|c|}{ Area } & \multicolumn{3}{|c|}{ Area } \\
\hline square inches & 6.4516 & $\begin{array}{l}\text { square } \\
\text { centimeters }\end{array}$ & $\begin{array}{l}\text { square } \\
\text { centimeters }\end{array}$ & 0.155 & square inches \\
\hline square feet & 0.092 & square meters & square meters & 10.7639 & square feet \\
\hline square yards & 0.836 & square meters & square meters & 1.20 & square yards \\
\hline square miles & 2.59 & $\begin{array}{l}\text { square } \\
\text { kilometers }\end{array}$ & $\begin{array}{l}\text { square } \\
\text { kilometers }\end{array}$ & 0.39 & square miles \\
\hline acres & 0.404 & Hectares & hectares & 2.471 & acres \\
\hline \multicolumn{3}{|c|}{ Mass (weight) } & \multicolumn{3}{|c|}{ Mass (weight) } \\
\hline ounces & 28.35 & Grams & grams & 0.0352 & ounces \\
\hline pounds & 0.453 & Kilograms & kilograms & 2.2046 & pounds \\
\hline short ton & 0.907 & metric ton & metric ton & 1.10 & short ton \\
\hline \multicolumn{3}{|c|}{ Volume } & \multicolumn{3}{|c|}{ Volume } \\
\hline fluid ounces & 29.57 & Milliliters & milliliters & 0.03 & fluid ounces \\
\hline quarts & 0.95 & Liters & liters & 1.057 & quarts \\
\hline gallons & 3.79 & Liters & liters & 0.26 & gallons \\
\hline cubic feet: & 0.03 & cubic meters & cubic meters & 35.3147 & cubic feet \\
\hline cubic yards & 0.76456 & cubic meters & cubic meters & 1.308 & cubic yards \\
\hline \multicolumn{3}{|c|}{ Temperature } & \multicolumn{3}{|c|}{ Temperature } \\
\hline $\begin{array}{r}\text { Fahrenheit } \\
\vdots \\
\vdots \\
\end{array}$ & $\begin{array}{l}\text { subtract } 32 \\
\text { then } \\
\text { multiply by } \\
5 / 9 \text { ths }\end{array}$ & Celsius & Celsius & $\begin{array}{l}\text { multiply by } \\
9 / 5 \text { ths, then } \\
\text { add } 32\end{array}$ & Fahrenheit \\
\hline \multicolumn{3}{|c|}{ Energy } & \multicolumn{3}{|c|}{ Energy } \\
\hline $\begin{array}{c}\text { kilowatt hour } \\
\vdots \\
\end{array}$ & 3,412 & $\begin{array}{l}\text { British thermal } \\
\text { unit }\end{array}$ & $\begin{array}{l}\text { British thermal } \\
\text { unit }\end{array}$ & 0.000293 & kilowatt hour \\
\hline kilowatt $\vdots$ & 0.948 & $\begin{array}{l}\text { British thermal } \\
\text { unit per second }\end{array}$ & $\begin{array}{l}\text { British thermal } \\
\text { unit per second }\end{array}$ & 1.055 & kilowatt \\
\hline \multicolumn{3}{|c|}{ Force } & \multicolumn{3}{|c|}{ Force } \\
\hline $\begin{array}{l}\text { pounds per } \\
\text { square inch }\end{array}$ & 6.895 & Kilopascals & kilopascals & $\begin{array}{l}1.4504 \mathrm{x} \\
10^{-4}\end{array}$ & $\begin{array}{l}\text { pounds per } \\
\text { square inch }\end{array}$ \\
\hline
\end{tabular}




\subsection{ADMNISTRATIVE INFORMATION}

This chapter provides administrative information, a description of the proposed delisting action, and a statement of need/justification.

\subsection{NAME OF PETITIONER}

United States Department of Energy, Richland Operations Office

Richland, Washington

\subsection{CONTACTS}

For additional information, contact:

Name Title $\quad$ Telephone Number

J. E. Rasmussen Director (509) 376-5441

Office of Environmental Assurance, Permits, and Policy

Mailing address for contact:

U.S. Department of Energy, Richland Operations Office (DOE-RL)

P. O. Box 550

Richland, Washington 99352.

\subsection{FACILITY NAME AND LOCATION}

200 Area Effluent Treatment Facility (ETF)

200 East Area

Hanford Facility, Richland, Washington.

The single identification number issued to the Hanford Facility by the U.S. Environmental Protection Agency (EPA) and the Washington State Department of Ecology (Ecology) is EPA/State Identification Number WA7890008967.

\subsection{DESCRIPTION OF PROPOSED DELISTING ACTION}

The proposed delisting action requests modification of the existing ETF Final Delisting to expand the universe of multi-source leachate (F039) that can be treated at ETF and ultimately land disposed. Multisource leachate is expected to be generated as a result of mixed waste land disposal operations conducted in support of cleanup activities on the Hanford Facility. The original ETF Delisting Petition requested that 71.9 million liters per year of treated effluent bearing the designation 'F001' through 'F005', and/or 'F039' that is derived from 'F001' through 'F005' waste, be delisted. At the beginning of the delisting process, only 242-A Evaporator process condensate (PC) and two aqueous waste streams from the Plutonium-Uranium 48 Extraction (PUREX) Facility were identified for treatment at ETF. On June 13, 1995, EPA published the 
final rule (Final Delisting) that excluded 71.9 million liters per year of ETF effluent from "being listed as hazardous wastes" [60 Federal Register (FR) 31115 now promulgated in 40 Code of Federal Regulations (CFR 261)].

The ETF, which is located in the 200 East Area of the Hanford Facility (Figure 1-1), began waste management operations in November of 1995. The ETF is a Resource Conservation and Recovery Act (RCRA) of 1976 permitted treatment and storage unit that will be used to manage multi-source leachate generated from future mixed waste land disposal operations. This delisting modification document, therefore, is a request to remove language from the Final Delisting that restricts the multi-source leachate that can be managed at ETF to 'F039' that is derived from 'F001' through 'F005' waste. The basis for this modification request is treatment knowledge gained from ETF operations, surrogate testing performed in support of the original delisting effort (DOE/RL-92-72), and information available from manufacturers for equipment used in the ETF.

The scope of the ETF delisting modification is limited to the addition of constituents associated with multisource leachate generated from land disposal operations. The multi-source leachate waste streams to be managed at the ETF will result from RCRA-compliant/permitted land disposal operations. This leachate will be transferred to the Liquid Effluent Retention Facility (LERF) or ETF, which are both RCRA-compliant waste management units. Following treatment at ETF, the effluent will be subject to the conditions of the Washington State Waste Discharge Permit (ST 4500) as well as the conditions of the Final Delisting. This delisting modification document, therefore, applies the upfront delisting process that depends on the ability of the resulting treated effluent to meet the concentration levels specified in the Final Delisting.

This delisting modification document demonstrates that multi-source leachate will be treated adequately so that the resulting effluent from the ETF will no longer require management under RCRA Subtitle C or the State of Washington Hazardous Waste Management Act (HWMA). This determination will apply to hazardous waste and dangerous waste. Dangerous waste is waste regulated under the HWMA. This demonstration will be accomplished by grouping the multi-source leachate constituents, as well as a very broad list of hazardous and dangerous constituents into treatability groups of like chemistry. Consistent with the approach taken in the original petition, the approach detailed in this document shows that effective treatment of these groups can be achieved. In addition, the sampling/analysis approach to be employed is detailed. The demonstration shows how the broad list of constituents, other than F001 through F005 constituents; will be below delisting levels following treatment. Given this strategy, no bench-scale, surrogate, or other testing is proposed.

In summary; all aspects of waste management before and following generation of multi-source leachate (Figure 1-2) will be govemed by associated regulations and permits. The control imposed by these regulations and permits on the management of land disposed waste and the generation and subsequent management of multi-source leachate further justifies delisting.

\subsection{STATEMENT OF NEED/JUSTIFICATION}

To support the current Hanford Facility mission, disposal capacity for mixed waste generated during cleanup is required. In addition, if proper public comment and stakeholder participation determines offsite multi-source leachate can be treated at the ETF, this delisting petition modification will support treatment of offsite generated multi-source leachate. When disposed, landfilled mixed waste will meet the RCRA and HWMA land disposal restriction (LDR) standards. RCRA-compliant trenches currently exist for mixed waste disposal. Disposal of waste in these trenches will result in the generation of multi-source leachate. This newly generated waste will receive the designation F039. Following treatment at LERF and ETF, the 
resulting effluent will be discharged to the State-Approved Land Disposal Site (SALDS) north of the 200 West Area. However, because the treated effluent also will be considered a hazardous waste and dangerous waste under the derived-from rule, and the RCRA and HWMA regulations prohibit the placement of liquid hazardous waste (40 CFR 264.314) and liquid dangerous waste [Washington Administrative Code (WAC) 173-140(4)(b)] on land, this effluent must be delisted before being discharged to the SALDS. The effluent also must meet the discharge criteria of ST 4500 and the Final Delisting (40 CFR 261, Appendix IX, Table 2).

The current delisting for the ETF addresses waste streams bearing the designation ' $\mathrm{F} 001$ ' through 'F005', and/or ' $\mathrm{F} 039$ ' that is derived from ' $\mathrm{F} 001$ ' through ' $\mathrm{F} 005$ ' waste. However, current mixed waste inventories on the Hanford Facility, when disposed, will generate multi-source leachate having a much broader spectrum of constituents than the F001 through F005 covered by the current delisting. Broad spectrum constituent mixed waste disposal operations are budgeted to begin in fiscal year 2000. To support this activity, effluent generated from treating leachate must be delisted in accordance with the EPA regulations promulgated under the authority of RCRA found in 40 CFR 260.20 and 260.22, as well as the HWMA found in WAC 173-303-072(1) and -072(4).

\subsection{CERTIFICATION STATEMENT}

I certify under penalty of law that I have personally examined and am familiar with the information submitted in this demonstration and all attached documents, and that, based on my inquiry of those individuals immediately responsible for obtaining the information, I believe that the submitted information is true, accurate, and complete. I am aware that there are significant penalties for submitting false information, including the possibility of fine and imprisonment.
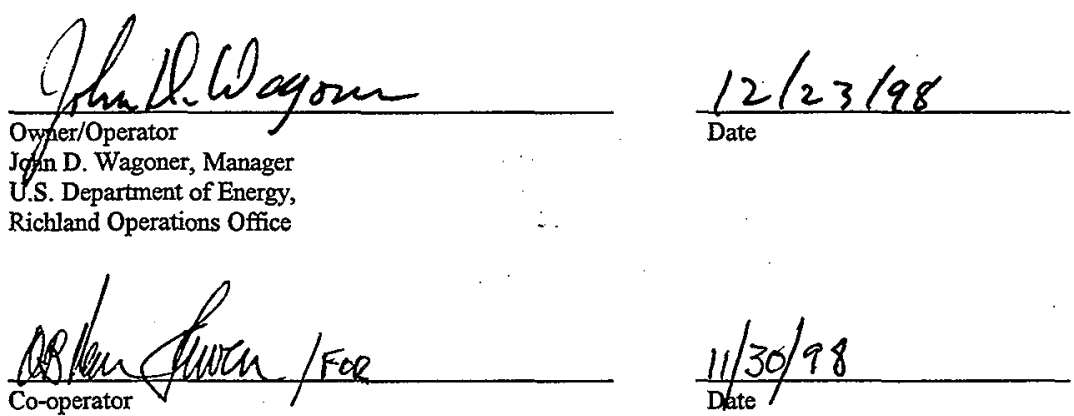

R. D. Hanson, President and Chief Executive Officer

Fluor Daniel Hanford, Inc. 
DOE/RL-98-62, Rev. 0

$11 / 98$

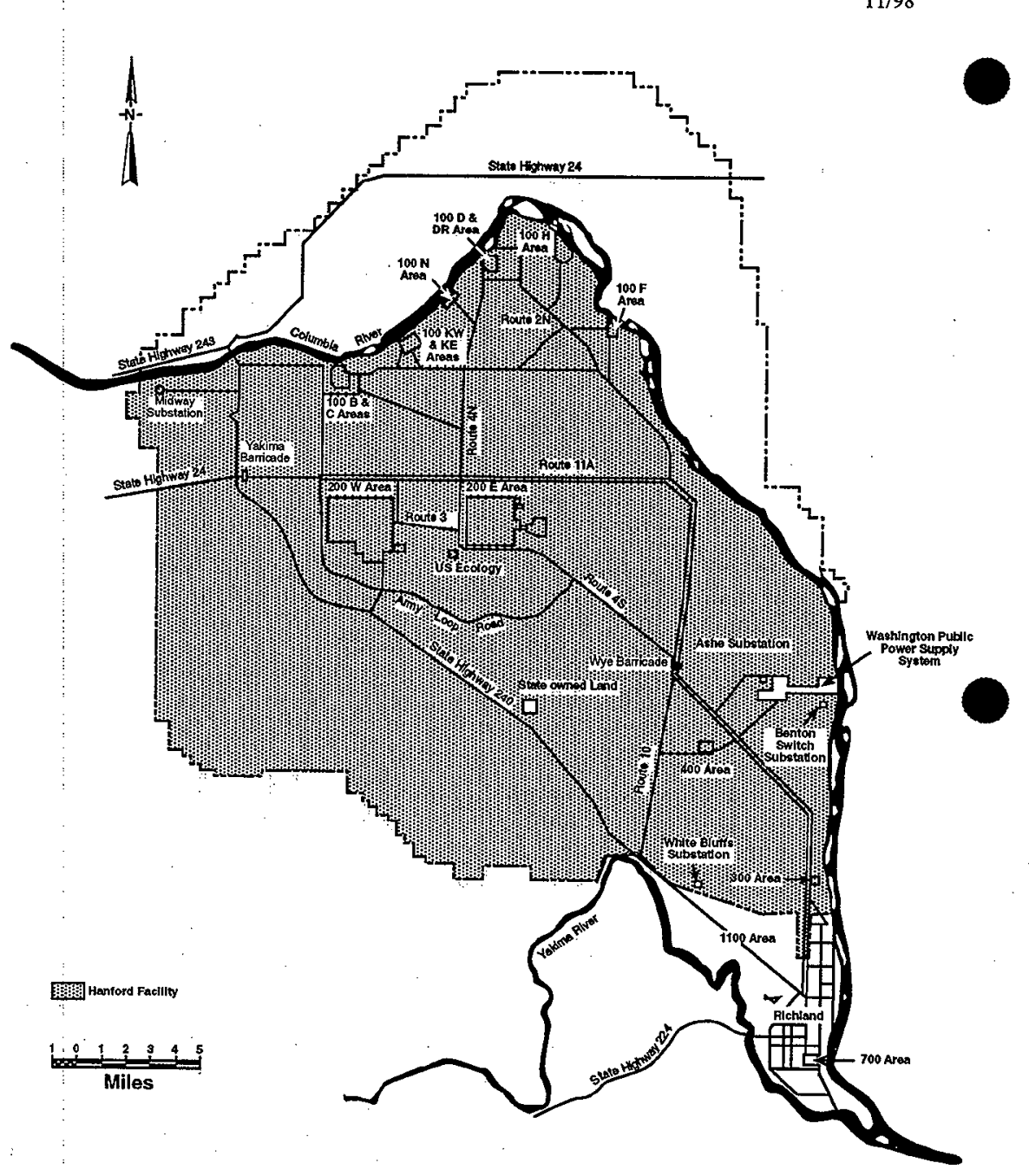

H97020271.1

Figure 1-1. Hanford Facility. 
DOE/RL-98-62, Rev. 0

$11 / 98$

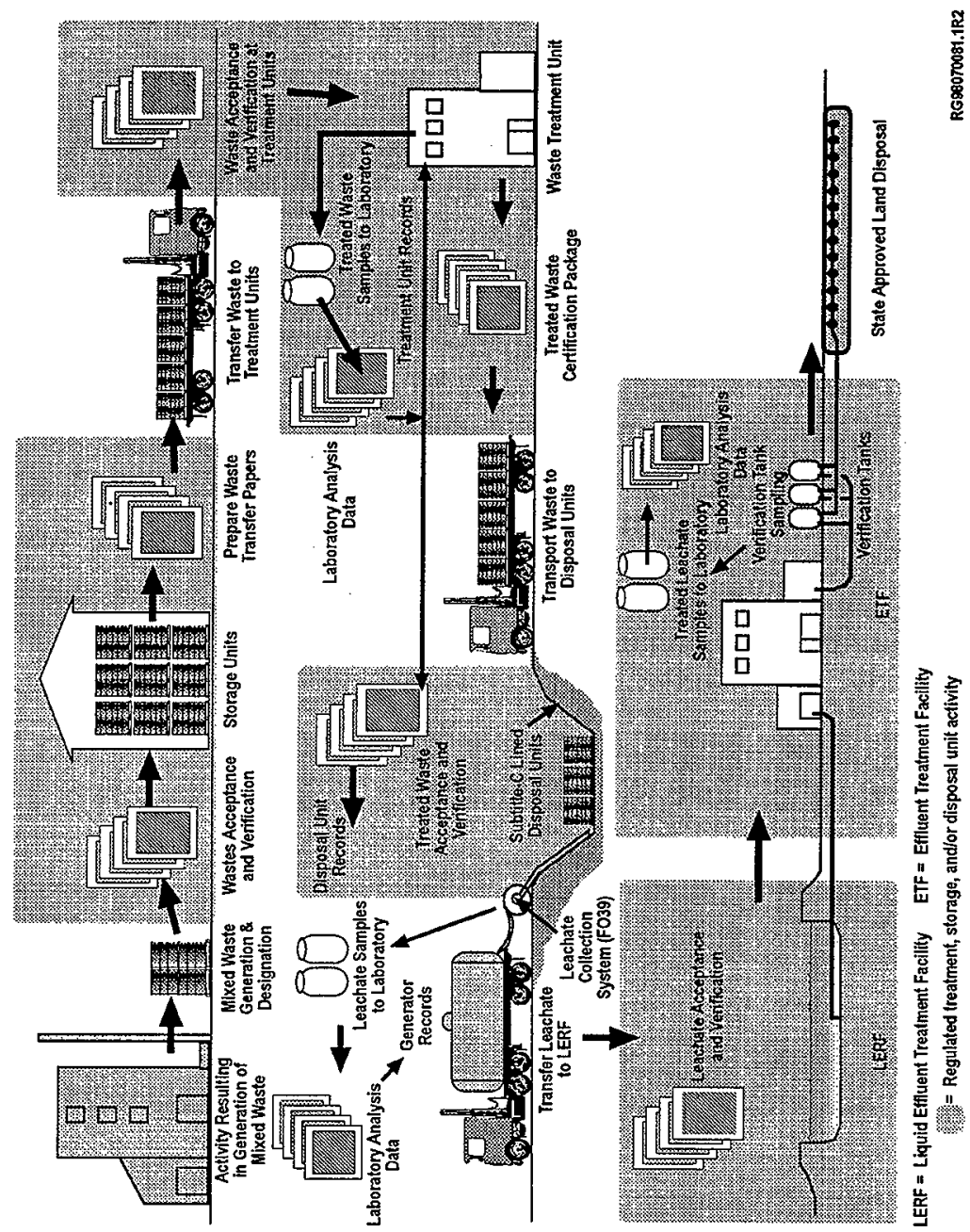

Figure 1-2. F039 Leachate Generation and Disposition. 
DOE/RL-98-62, Rev. 0

11/98

This page intentionally left blank. 
2

3

4

Leachate (liquids that have percolated through land disposed waste) resulting from the disposal of more than one restricted waste classified as hazardous (RCRA Subpart D) and/or dangerous (WAC 173-303) are said to be multi-source. Multi-source leachates are newly generated waste designated with the F039 waste number per WAC 173-303-082 (40 CFR 261.31). By regulation, only the F039 listed waste number can be applied to the newly generated multi-source leachate. Waste numbers associated with the disposed waste in a landfill from which the leachate is generated are not carried forward.

\subsection{LEACHATE GENERATION}

As of this submittal, multi-source leachate has not been generated on the Hanford Facility. However, the Environmental Restoration Disposal Facility (ERDF) located in the 600 Area has generated leachate under Comprehensive Environmental Response, Compensation, and Liability Act (CERCLA) of 1980 authority. The ERDF adopted the RCRA/HWMA waste designation and LDR regulations as part of their applicable or relevant and appropriate requirements (ARARs). The single-source leachate generated from ERDF operations currently is being sent to ETF for treatment under CERCLA authority; therefore, ETF has operational history at treating leachates. The ERDF leachate is expected to become multi-source in the future and will be delisted under CERCLA authority.

Beginning in fiscal year 1999, disposal operations are to begin in lined RCRA-compliant disposal units (Section 2.1.1). Initial disposal operations will be limited to waste that meets treatment standards for characteristic waste, F001 through F005 listed constituents (nonspecific source waste), and state-only requirements (WAC 173-303). The leachate generated from these disposal operations is acceptable for treatment at ETF under the Final Delisting. However, beginning in fiscal year 2000, disposal operations will be expanded to include waste that meets treatment standards for other listed constituents managed on the Hanford Facility (e.g., other F, P, and U designated waste). The multi-source leachate generated from these operations also will be treated at the ETF.

\subsubsection{Leachate Sources}

Currently there are only two lined RCRA-compliant disposal trenches where multi-source leachate will be generated, trenches 31 and 34 of the 218-W-5 Low-Level Burial Grounds (DOE/RL-88-20). Additional lined RCRA-compliant disposal trenches will be constructed on an as-needed basis to accommodate mixed waste disposal volumes.

Construction of trenches 31 and 34 was completed in 1995. Each trench can hold between 22,800 to 52,760 cubic meters of LDR-compliant mixed waste (based on 'containerized' and 'bulk' waste respectively). The trenches meet minimum technological requirements for landfills. The liners in the trenches are made from high-density polyethylene (HDPE), a standard commercially available liner material that provides a high resistance to chemical degradation. Precipitation will percolate through the disposed waste and form the leachate. This leachate will be collected in sumps embedded in the trench bottom and will be pumped periodically into accumulation tanks. Samples of the leachate will be taken for analysis according to a schedule based on meeting generator requirements and requirements imposed through the waste analysis plan of the treatment unit receiving the waste.

Other sources of F039 designated waste could include, but are not limited to, offsite generators, additional disposal sites outside the 200 West and 200 East Areas, and waste generated from application of the derived from rule and/or mixture rale. 


\subsubsection{Mixed Waste Disposal}

Important factors to this delisting modification include the amount of mixed waste in storage and the acceptance criteria for the disposal units on the Hanford Facility.

\subsubsection{Mixed Waste Volumes}

Mixed waste currently in storage on the Hanford Facility consists of debris, solar evaporation basin solids [from 183-H Solar Evaporation Basins (183-H Solar Evaporation Basins Closure/Post-Closure Plan, Hanford Facility RCRA Permit, Dangerous Waste Portion, Attachment 11)] and miscellaneous waste (e.g., labpacks, soils, wastewater treatment secondary solids). Currently, there are approximately 8,600 cubic meters of solid mixed waste residing in storage (DOE/RL-98-09).

- Debris consists of various debris constituents including metals (e.g., pipe, pumps, sheet metal), building rubble (e.g., concrete, brick, roofing), and organic/carbonaceous materials (e.g., wood, plastic, paper, asphalt). Currently 3,100 cubic meters of debris are stored at Central Waste Complex (CWC) (DOE/RL-91-17). The waste is packaged in 208-liter containers or various sized boxes. The primary generating units for these waste types are the Single-Shell and Double-Shell Tank Systems (DOE/RL-88-21). The waste is designated with listed waste numbers F001 through F005. Debris from other generating units have various other waste numbers (both characteristic and listed).

- The 183-H Solar Evaporation Basins solids consist of solidified basin liquids, crystalline solids, sludges, and particulates (sandblast grit) from closure activities. Currently, there are 3,500 cubic meters of this waste type stored at CWC. The waste is designated with listed waste constituents P029, P030, P098, P106, P120, and U123. The concentrations of these constituents in the waste is very low (i.e., below LDR concentration limits) or nondetectable.

- The CWC stores several smaller volume, unique mixed waste streams (e.g., inorganic and organic labpacks, elemental lead and mercury, inorganic and organic solids/particulates, and batteries). There are approximately 105 different waste numbers associated with these waste streams. Additional listed and characteristic waste numbers are expected to be received from the receipt of forecasted waste. The CWC Part A, Form 3, Permit Application (DOE/RL-88-21) reflects this broad range of waste numbers by including all $D, P$, and $U$ numbers, and applicable $F$ numbers.

The volume of waste associated with each waste number currently being managed at CWC is provided in Table 2-1. Federal waste numbers F001 through F005, P029, P030, P098, P106, P120, U123, D001, D007, D008; and state-only waste numbers WTO2, WP02, and WT01 are the most significant on a waste volume basis.

An additional 63,750 cubic meters of solid mixed waste has been forecasted to be generated through fiscal year 2032 (DOE/RL-98-09). The significant waste numbers applicable to the forecasted waste are anticipated to be D001 through D043 and F001 through F005.

\section{1:2.2 Mixed Waste Disposal Acceptance Criteria}

All waste accepted at lined RCRA-compliant disposal trenches must meet LDR treatment standards (40 CFR 268 and WAC 173-303-140). In addition to these requirements, there also are safety-based and environmentally-based limits on the radionuclide concentrations of the disposed waste. 
1 The federal LDR treatment standards take three basic forms: "total waste standards"

2 [40 CFR 268.40(a)(1)], "waste extract standards" [40 CFR 268.40(a)(2)], or the "technology standard"

3 [40 CFR 268.40(a)(3)]. In addition to these standards, much of the debris waste is, and will be, treated

4 using one or more of the "Alternative Treatment Standards for Hazardous Debris" (40 CFR 268.45).

5 Mixed waste containing polychlorinated biphenyls (PCBs) under the Toxic Substances Control Act

6 (TSCA) of 1976 jurisdiction will be treated meeting the standards specified in 40 CFR 761. The state-only

7 LDR treatment standards are nonspecific with respect to treatment levels or specified treatment

8 technologies. The treated waste however must be treated, detoxified, or otherwise processed to remove or

9 reduce the harmful properties or characteristics. For waste that has "concentration-based" treatment

10 standards for specific hazardous constituents under 40 CFR 268, the waste must be tested in accordance

11 with 40 CFR 268. For waste treated to "technology standards", process knowledge must demonstrate the

12 treatment was done in a "well designed and well operated" manner.

For waste requiring treatment before disposal, the following treatment paths have been developed to disposition the waste.

- Non-thermal Treatment-Uses treatment technologies that do not depend on elevated temperatures to obtain compliant and successful treatment. Treatment technologies include, but are not limited to, stabilization, chemical reduction, deactivation, macroencapsulation (elemental lead and hazardous debris), neutralization, and mercury amalgamation. Inorganic hazardous constituents normally are treated by non-thermal means. This includes, but is not limited to, metals, corrosives, some ignitables and reactives, and certain hazardous debris.

Currently, non-thermal treatment is being pursued onsite. These treatment units are either under interim status or are in the process of obtaining RCRA final status permits.

- Thermal Treatment--Uses treatment technologies that depend on elevated temperatures to obtain successful treatment. Treatment technologies include, but are not limited to, combustion, incineration, vitrification, gasification, steam reforming, and roasting/retorting. Organic hazardous constituents normally are treated by thermal means. This includes, but is not limited to, organics (D012 through D043), some ignitables and reactives, listed organic constituents, and hazardous debris containing organic/carbonaceous waste.

Treatment technologies are and will be matched with the specific hazardous waste constituents required (i.e., technology standard) or recommended [best demonstrated available technology (BDAT)]. Based on current regulations, approximately 60 percent of the disposed mixed waste will be treated by non-thermal treatment technologies, 30 percent by thermal treatment technologies, and 10 percent not treated because the waste either meets LDRs as-generated or administrative processes have authorized the disposal of specific waste streams. The major treated waste form to be disposed is macroencapsulated inorganic hazardous debris (mostly stainless steel) originating from Single-Shell and Double-Shell Tank System activities.

\subsubsection{Leachate Collection}

Infiltration of precipitation (e.g., rain, snowmelt) through the disposed waste will be the primary contributor to the leachate generation rate in the disposal areas. Precipitation will enter the disposal areas through the exposed surface area, slowly migrate downward through the waste to the primary lining, and flow along the sloped lining to one or more leachate collection sumps designed into the low points of each disposal trench. At a set operational level, these sumps will be pumped into an accumulation tank. When enough leachate has accumulated in a tank, the leachate will be transported by tanker truck to LERF or ETF. 
Current planning assumes that the majority of the waste to be disposed in lined RCRA-compliant disposal trenches will be containerized (e.g., steel drums and boxes, HDPE macroencapsulated forms). During initial operations, infiltration of precipitation will not come into contact with the containerized treated waste. Contact with the treated waste would not occur until some of the corrodible containers are breached (10 years is assumed based on a 0.13 millimeter per year corrosion rate). However, bulk waste disposal will be an option at the disposal trenches; therefore, precipitation would readily come into contact with this portion of the disposed waste.

\subsubsection{Expected Leachate Volumes and System Capacities}

The quantity of leachate generated from a given disposal trench is expected to vary over the life of the trench depending on the exposed surface area, amount of waste disposed, solar evaporation rates, and yearly precipitation amounts. The leachate flow would be greatest at inception of disposal operations and progressively decrease as filling occurs until the burial ground is capped and closed, at which time leachate production would become fairly steady state. However, leachate generation can increase significantly during the winter months and/or during a heavy precipitation event. Based on data compiled from pumping accumulated precipitation from the existing lined disposal areas, approximately 120 liters of leachate per square meter of open disposal surface area initially can be expected each year. It is assumed that the maximum effective open disposal surface area would not exceed 90,000 square meters in a given year; therefore, the maximum yearly leachate generation rate would be approximately $10,800,000$ liters.

The currentleachate accumulation tanks associated with trenches 31 and 34 are 37,850 liters each (this capacity is not indicative of all future leachate storage tanks anticipated for use). This volume is effective for an average leachate production rate of approximately 4,100 liters per day (1,500,000 liters per year). However, peak precipitation events significantly could elevate the daily generation rate. The theoretical maximum daily rate is 416,400 liters based on the 25 -year 24 -hour precipitation event. At this generation rate, the accumulation tanks would need to be emptied every 2 hours and the leachate shipped in 18,900- to 28,400-liter tankers to LERF.

\subsection{LEACHATE MANAGEMENT}

As stated previously, multi-source leachates are newly generated waste designated with the F039 listed waste number per WAC 173-303-082 (40 CFR 261.31). Waste numbers associated with the disposed waste in a landfill from which the leachate is generated do not carry forward unless the waste exhibits a hazardous characteristic that is not specifically addressed by the F039 listed waste number (i.e., ignitable, corrosive, or reactive). The F039 LDR treatment standard consists of approximately 200 hazardous constituents. The regulations do not require that all hazardous constituents specified on the list need to be monitored; instead, the generator is required to identify only those F039 hazardous constituents that are reasonably expected to be present in the leachate at the point of generation above the concentration levels identified in 40 CFR 268.40 .

Leachate generated from lined RCRA-compliant disposal trenches on the Hanford Facility initially will reside in accumulation tanks managed per the generator provisions (40 CFR 262.34 and WAC 173-303-200). The leachate will be designated with waste number F039 and applicable F039 hazardous constituents reasonably expected to be present in the waste at the point of generation will be determined. Generator sample analysis plans will be used where applicable to determine the appropriate hazardous constituents to be monitored. The frequency of sampling the accumulated multi-source leachate will be based primarily on three factors to meet generator acceptable knowledge requirements. The three factors are: the amount of precipitation that percolates through land disposed waste, the likeliness of the 
precipitation to contact land disposed waste, and the volume of waste disposed for any given waste number.

Further waste characterization may be required by LERF/ETF to meet waste acceptance requirements specified in their waste analysis plan (DOE/RL-97-03)

\subsubsection{Leachate Baseline Data}

To provide a baseline for determining which leachate constituents are inherent to the disposal trench as opposed to those associated with the disposed waste, samples of liquids from the two unused lined RCRA Subtitle-C disposal units were obtained during September 1996. The liquids originated from precipitation run-off and were removed using the leachate removal systems.

Analysis performed on the samples included the following parameters: inductively coupled plasma (ICP) metals, anions, volatile organics and aromatics, semivolatile organics and aromatics, pesticides, polychlorinated biphenyls (PCBs), and radiological screening. From the analysis, all detectable constituents were significantly below (by several orders of magnitude) LDR regulatory levels, and no organic constituents were detected. Table 2-2 identifies the hazardous constituents that showed concentrations in the baseline samples above the minimum detection levels (MDL).

\subsubsection{Leachate Constituents}

For the purpose of identifying the constituents to be addressed by this delisting modification document, an exhaustive evaluation of several hazardous constituent lists was performed. The lists considered are as follows:

- 40 CFR 261 Subpart C - Characteristics of Hazardous Waste

- 40 CFR 261 Subpart D - Lists of Hazardous Wastes

- Hazardous wastes from non-specific sources (as applicable)

- Discarded commercial chemical products, off-specification species, container residues, and spill residues thereof

- 40 CFR 261 Appendix VIII, Hazardous Constituents

- 40 CFR 268.40, F039

- 40 CFR 268.48, Universal Treatment Standards

- Constituents specified in the document Petitions to Delist Hazardous Wastes, A Guidance Manual (EPA 530/R-93/007)

- Oil and Grease

- Cyanide, total

- Cyanide, reactive

- Nickel

- Sulfide, total

- Sulfide, reactive

- Acetone

- Ethylbenzene

- Isophorone

- 4-methyl-2-pentanone

- Styrene

- Xylenes

- Docket Report on Health-Based Levels and Solubilities Used in the Evaluation of Delisting Petitions, Submitted Under 40 CFR 260.20 and 260.22 (EPA 1994)

49 Upon defining the constituent lists to be considered in support of this delisting modification effort, it 
1 became obvious that not all of the identified constituents would be present in the multi-source leachate

2 waste streams to be treated. Figure 2-1 details the logic used to evaluate each constituent from the lists

3 identified previously. The following is a description of each decision point:

A1: This activity combines the various constituent source lists together into one list. Equivalent constituents from each list were combined and presented once in the list. An attempt was made to identify Chemical Abstract Service (CAS) numbers for those constituents that were not represented by one from the source lists:

A2: This activity removes all the "Not Otherwise Specified" (NOS) general constituent classes from the consolidated constituent list (A1), and sends these to activity (A3) for re-assignment.

A3: To allow evaluation of the NOS generic constituent classes, a specific representative constituent is assigned to the generic class. This representative constituent is chosen from the consolidated constituent list (A1). If the $\mathrm{Al}$ list does not have a representative constituent for a generic class, a representative constituent is chosen from other sources and the source is referenced.

A4: This activity identifies compounds that have the same regulated hazardous constituents, and assigns the regulated constituent CAS number to the compound. This activity is similar to the A3 activity, but instead focuses on the various salts of the primary constituent.

A5: This activity identifies those constituents that can be eliminated from consideration based on professional judgment. Many of the constituents residing in the consolidated constituent list are unlikely to be associated with waste generating activities on the Hanford Site or other DOE sites that are considering disposing mixed waste on the Hanford Site (e.g., pesticides, fungicides, pharmaceuticals). Furthermore, because this petition applies to multi-source leachate, organic compounds insoluble in water should not be considered for analysis. The ERDF leachate delisting petition (DOE/RL-98-47) was consulted with regard to this analysis. In the subject document, Table A9 was reviewed and determined to be applicable to this petition. The professional judgment decisions detailed here were based on searches of various hazardous substance databases (including applicable material safety data sheets), and by using 40 CFR 264 Appendix IX, Ground Water Monitoring List, in the decisionmaking.

A6: This activity identifies those hazardous constituents that reasonably are not likely to be present in the waste being disposed because of the regulatory specified treatment method(s) performed on the waste before disposal. Many of the constituents residing in the consolidated constituent list are identified by a specific listed waste number that are treated by the specified technology-based standard of CMBST (combustion) or $A D G A S$ (venting of compressed gas). These technologies destroy or remove the constituent(s) from the waste before disposal; therefore, it is reasonable to state that the constituents would not be present in the disposed waste. The constituents on the consolidated constituent list identified with the $C M B S T$ and $A D G A S$ technology-based standard are removed from further consideration.

A7: This activity denotes those constituents that have a potential to be found in the multi-source leachate waste streams. These constituents will be evaluated for treatment effectiveness at ETF.

The results of the evaluation performed are detailed in Table 2-3. This table identifies the constituents that are addressed by this delisting modification document.

\subsubsection{Generator Sample and Analysis Plans}

For multi-source leachate managed pursuant to this delisting modification, the approach to identifying and managing the constituents that have a potential to be found in the multi-source leachate waste stream will 
be through the use of sample and analysis plans (SAPs). These SAPs generally will be based on the following:

- Establish baseline knowledge of the natural occurring constituents in the disposal unit that migrate to the leachate

- Periodically assess the type and quantity of constituents being disposed that have a potential to be found in the multi-source leachate waste stream and apply this knowledge to the list of constituents being analyzed

- Assess the physical form and packaging of the waste being disposed

- Assess the receiving treatment, storage, and disposal (TSD) unit's waste acceptance criteria requirements and incorporate those applicable requirements into the SAPs

- Periodic assessments to determine if changes to the leachate sampling and analysis requirements are needed as the disposal unit matures. The assessment will include evaluating multi-source leachate analytical result trends and accounting for reduction in leachate generation as the disposal units are filled and monitored during post-closure operations. 


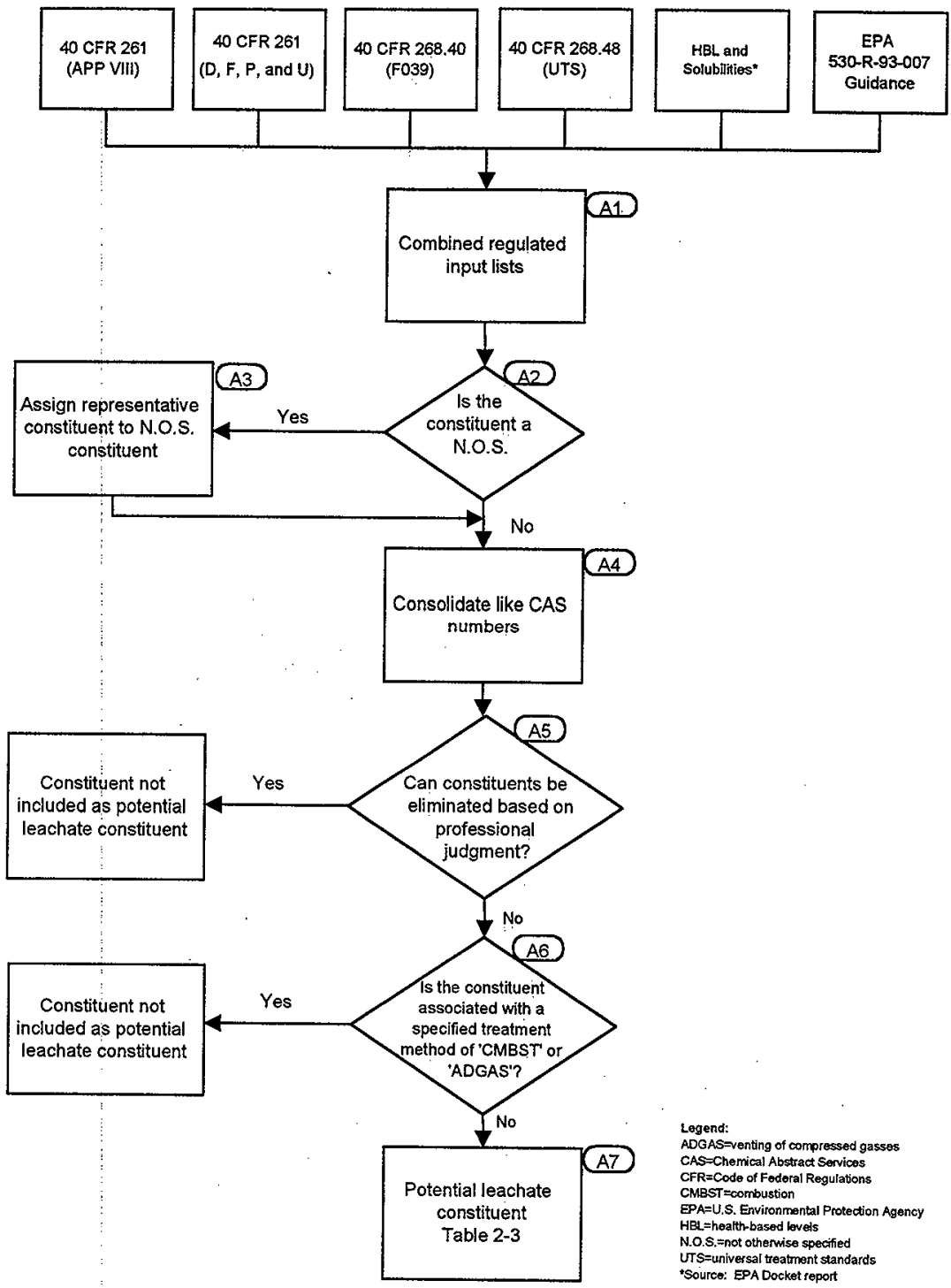

Figure 2-1. Determination of Potential Leachate Constituents. 
Table 2-1. Waste Volume ${ }^{[1]}$

\begin{tabular}{|c|c|c|c|c|}
\hline $\begin{array}{l}\text { Waste } \\
\text { number }\end{array}$ & Waste number description & $\begin{array}{l}\text { Waste volume } \\
\text { containing } \\
\text { subject waste } \\
\text { number }\left(\mathrm{m}^{3}\right)\end{array}$ & $\begin{array}{l}\text { Package count } \\
\text { containing } \\
\text { subject waste } \\
\text { number }\end{array}$ & $\begin{array}{l}\text { Waste volume } \\
\text { percent of each } \\
\text { waste number }\end{array}$ \\
\hline F003 & Organic solvents & $3,336.90$ & 5,066 & 38.8 \\
\hline D001 & Ignitable & $3,189.40$ & 11,262 & 37.1 \\
\hline F005 & Organic solvents & $3,121.10$ & 4,178 & 36.3 \\
\hline F001 & Organic solvents & $3,078.40$ & 3,966 & 35.8 \\
\hline $\mathrm{F} 002$ & Organic solvents & $3,073.00$ & 3,975 & 35.7 \\
\hline F004 & Organic solvents & $2,913.00$ & 3,383 & 33.9 \\
\hline WT02 & Toxic dangerous wastes DW & $2,896.00$ & 9,215 & 33.7 \\
\hline $\mathrm{P} 098$ & Potassium cyanide & $2,885.40$ & 9,059 & 33.6 \\
\hline U123 & Formic acid & $2,885.20$ & 9,058 & 33.5 \\
\hline P029 & Copper cyanide & $2,885.00$ & 9,057 & 33.5 \\
\hline $\mathrm{P} 030$ & Cyanide total & $2,885.00$ & 9,057 & 33.5 \\
\hline P106 & Sodium cyanide & $2,885.00$ & 9,057 & 33.5 \\
\hline P120 & Vanadium pentoxide & $2,885.00$ & 9,057 & 33.5 \\
\hline WP02 & \begin{tabular}{|l|} 
Persistent dangerous wastes \\
halogenated organic compounds DW
\end{tabular} & $2,232.00$ & 2,202 & 26.0 \\
\hline WT01 & Toxic dangerous wastes EHW & $1,701.50$ & 6,418 & 19.8 \\
\hline D008 & Lead & $1,617.60$ & 2,627 & 18.8 \\
\hline D007 & Chromium & $1,335.70$ & 3,627 & 15.5 \\
\hline D009 & Mercury & 330.0 & 699 & 3.8 \\
\hline D006 & Cadmium & 256.9 & 599 & 3.0 \\
\hline $\mathrm{D} 002$ & Corrosive & 231.6 & 654 & 2.7 \\
\hline WP01 & $\begin{array}{l}\text { Persistent dangerous wastes } \\
\text { halogenated organic compounds } \\
\text { EHW }\end{array}$ & 133.5 & 496 & 1.6 \\
\hline D005 & Barium & 90.2 & 338 & 1.0 \\
\hline $\mathrm{D} 010$ & Selenium & 89.9 & 334 & 1.0 \\
\hline D011 & Silver & 72.2 & 240 & 0.8 \\
\hline U031 & n-Butyl alcohol & 69.8 & 333 & 0.8 \\
\hline U108 & 1,4-Dioxane & 69.4 & 331 & 0.8 \\
\hline P022 & Carbon disulfide & 68.5 & 327 & 0.8 \\
\hline $\mathrm{P} 023$ & Chloroacetaldehyde & 68.5 & 327 & 0.8 \\
\hline U001 & Acetaldehyde & 68.5 & 327 & 0.8 \\
\hline U025 & Bis(2-Chloroethyl)ether & 68.5 & 327 & 0.8 \\
\hline U117 & Ethyl ether & 68.5 & 327 & 0.8 \\
\hline $\mathrm{U} 160$ & Methyl ethyl ketone peroxide & 68.5 & 327 & 0.8 \\
\hline $\mathrm{U} 213$ & Tetrahydrofuran & 68.5 & 327 & 0.8 \\
\hline U359 & 2-Ethoxyethanol & 56.9 & 271 & 0.7 \\
\hline D040 & Trichloroethylene & 56.1 & 270 & 0.7 \\
\hline D039 & Tetrachloroethylene & 54.3 & 262 & 0.6 \\
\hline $\mathrm{WSC} 2$ & Corrosivity & 48.3 & 227 & 0.6 \\
\hline D019 & Carbon tetrachloride & 48.2 & 221 & 0.6 \\
\hline
\end{tabular}


Table 2-1. Waste Volume $e^{[1]}$.

\begin{tabular}{|c|c|c|c|c|}
\hline $\begin{array}{l}\text { Waste } \\
\text { number }\end{array}$ & Waste number description & $\begin{array}{l}\text { Waste volume } \\
\text { containing } \\
\text { subject waste } \\
\text { number }\left(\mathrm{m}^{3}\right)\end{array}$ & $\begin{array}{l}\text { Package count } \\
\text { containing } \\
\text { subject waste } \\
\text { number }\end{array}$ & $\begin{array}{l}\text { Waste volume } \\
\text { percent of each } \\
\text { waste number }\end{array}$ \\
\hline W001 & PCBs & 47.0 & 157 & 0.5 \\
\hline D004 & Arsenic & 45.8 & 137 & 0.5 \\
\hline D035 & Methyl ethyl ketone & 41.8 & 125 & 0.5 \\
\hline D018 & Benzene & 39.6 & 168 & 0.5 \\
\hline D029 & 1,1-Dichloroethylene & 38.3 & 165 & 0.4 \\
\hline D043. & Vinyl chloride & 31.7 & 153 & 0.4 \\
\hline $\mathrm{D} 003$ & Reactive & 26.3 & 65 & 0.3 \\
\hline D028 & 1,2-Dichloroethane & 23.5 & 93 & 0.3 \\
\hline$\overline{\mathrm{D} 037}$ & Pentachlorophenol & 17.6 & 3 & 0.2 \\
\hline D022 & Chloroform & 17.0 & 63 & 0.2 \\
\hline $\mathrm{D} 026$ & Cresols Total & 15.2 & 14 & 0.2 \\
\hline D036 & Nitrobenzene & 14.4 & 50 & 0.2 \\
\hline D033 & Hexachlorobutadiene & 6.5 & 12 & 0.1 \\
\hline D038 & Pyridine & 5.0 & 24 & 0.1 \\
\hline D027 & 1,4-Dichlorobenzene & 4.6 & 3 & 0.1 \\
\hline D034 & Hexachloroethane & 4.4 & 2 & 0.1 \\
\hline D030 & 2,4-Dinitrotoluene & 4.4 & 20 & 0.1 \\
\hline U002 & Acetone & 2.9 & 14 & $<0.1$ \\
\hline U151 & Mercury & 2.9 & 14 & $<0.1$ \\
\hline U133 & Hydrazine & 2.9 & 11 & $<0.1$ \\
\hline $\mathrm{U} 239$ & Xylene total & 2.3 & 11 & $<0.1$ \\
\hline $\mathrm{U} 226$ & 1,1,1-Trichloroethane & 2.1 & 10 & $<0.1$ \\
\hline D012 & Endrin & 2.0 & 6 & $<0.1$ \\
\hline D03I & Heptachlor & 1.8 & 7 & $<0.1$ \\
\hline WP03 & $\begin{array}{l}\text { Polycyclic aromatic hydrocarbons } \\
\text { EHW }\end{array}$ & 1.7 & 8 & $<0.1$ \\
\hline U161 & Methyl isobutyl ketone & 1.5 & 7 & $<0.1$ \\
\hline $\mathrm{U} 154$ & Methanol & 1.5 & 7 & $<0.1$ \\
\hline U159 & Methyl ethyl ketone & 1.4 & 7 & $<0.1$ \\
\hline U080 & Methylene chloride & 1.2 & 6 & $<0.1$ \\
\hline $\mathrm{U} 006$ & Acetyl chloride & 1.1 & 4 & $<0.1$ \\
\hline D023 & o-Cresol & 1.0 & 5 & $<0.1$ \\
\hline U220 & Toluene & 1.0 & 5 & $<0.1$ \\
\hline U019 & Benzene & 0.9 & 4 & $<0.1$ \\
\hline U189 & Phosphorus sulfide & 0.9 & 3 & $<0.1$ \\
\hline U162 & Methyl methacrylate & 0.8 & 4 & $<0.1$ \\
\hline $\mathrm{U} 188$ & Phenol & 0.8 & 4 & $<0.1$ \\
\hline $\mathrm{P} 012$ & Arsenic trioxide & 0.6 & 3 & $<0.1$ \\
\hline $\mathrm{U} 112$ & Ethyl acetate & 0.6 & 3 & $<0.1$ \\
\hline D016 & 2,4,Dichlorophenoxyacetic acid & 0.4 & 2 & $<0.1$ \\
\hline U044 & Chloroform & 0.4 & 2 & $<0.1$ \\
\hline U056 & Cyclohexane & 0.4 & 2 & $<0.1$ \\
\hline
\end{tabular}


Table 2-1. Waste Volume ${ }^{[1]}$.

\begin{tabular}{|c|l|c|c|c|}
\hline $\begin{array}{c}\text { Waste } \\
\text { number }\end{array}$ & \multicolumn{1}{|c|}{ Waste number description } & $\begin{array}{c}\text { Waste volume } \\
\text { containing } \\
\text { subject waste } \\
\text { number }\left(\mathrm{m}^{3}\right)\end{array}$ & $\begin{array}{c}\text { Package count } \\
\text { containing } \\
\text { subject waste } \\
\text { number }\end{array}$ & $\begin{array}{c}\text { Waste volume } \\
\text { percent of each } \\
\text { waste number }\end{array}$ \\
\hline U121 & Trichlorofluoromethane & 0.4 & 2 & $<0.1$ \\
\hline U134 & Hydrogen fluoride & 0.4 & 2 & $<0.1$ \\
\hline U165 & Naphthalene & 0.4 & 2 & $<0.1$ \\
\hline U196 & Pyridine & 0.4 & 2 & $<0.1$ \\
\hline U218 & Thioacetamide & 0.4 & 2 & $<0.1$ \\
\hline U169 & Nitrobenzene & 0.4 & 2 & $<0.1$ \\
\hline U228 & Trichloroethylene & 0.4 & 2 & $<0.1$ \\
\hline U144 & Lead acetate & 0.4 & 2 & $<0.1$ \\
\hline U003 & Acetonitrile & 0.2 & 1 & $<0.1$ \\
\hline D020 & Chlordane & 0.2 & 1 & $<0.1$ \\
\hline D021 & Chlorobenzene & 0.2 & 1 & $<0.1$ \\
\hline F022 & Dioxins and furans & 0.2 & 1 & $<0.1$ \\
\hline P102 & Propargyl alcohol & 0.2 & 1 & $<0.1$ \\
\hline U004 & Acetophenone & 0.2 & 1 & $<0.1$ \\
\hline U057 & Cyclohexanone & 0.2 & 1 & $<0.1$ \\
\hline U103 & Dimethyl sulfate & 0.2 & 1 & $<0.1$ \\
\hline U170 & p-Nitrophenol & 0.2 & 1 & $<0.1$ \\
\hline U187 & Phenacetin & 0.2 & 1 & $<0.1$ \\
\hline U203 & Safrole & 0.2 & 1 & $<0.1$ \\
\hline U210 & Tetrachloroethylene & 0.2 & 1 & $<0.1$ \\
\hline U211 & Carbon tetrachloride & 0.2 & 1 & $<0.1$ \\
\hline
\end{tabular}

${ }^{(1)}$ Waste volumes represent what is currently in storage at the Central Waste Complex (current as of 9/98) and represent a subset of the waste numbers allowed for storage based on the Central Waste Complex Part A, Form 3, permit application.

$\mathrm{DW}=$ dangerous waste .

$\mathrm{EHW}=$ extremely hazardous waste.

$\mathrm{m}^{3}=$ cubic meters. 
Table 2-2. Baseline Constituents Above Method Detection Limits.

\begin{tabular}{|c|c|c|c|}
\hline F039 Constituent & $\begin{array}{c}\text { Measured } \\
\text { Concentration }\end{array}$ & $\mathrm{MDL}$ & $\begin{array}{c}\text { LDR Concentration } \\
\text { Limit }\end{array}$ \\
\hline \multicolumn{4}{|l|}{ Metals } \\
\hline Antimony & $1.0 \mathrm{ug} / \mathrm{L}$ & $0.30 \mathrm{ug} / \mathrm{L}$ & $1,900 \mathrm{ug} / \mathrm{L}$ \\
\hline Arsenic & $20.1 \mathrm{ug} / \mathrm{L}$ & $0.40 \mathrm{ug} / \mathrm{L}$ & $1,400 \mathrm{ug} / \mathrm{L}$ \\
\hline Barium & $32.5 \mathrm{ug} / \mathrm{L}$ & $0.20 \mathrm{ug} / \mathrm{L}$ & $1,200 \mathrm{ug} / \mathrm{L}$ \\
\hline Chromium & $8.7 \mathrm{ug} / \mathrm{L}$ & 1.10 ug $/ \mathrm{L}$ & $2,770 \mathrm{ug} / \mathrm{L}$ \\
\hline Copper & $9.5 \mathrm{ug} / \mathrm{L}$ & $0.40 \mathrm{ug} / \mathrm{L}$ & Non specified \\
\hline Lead & $1.9 \mathrm{ug} / \mathrm{L}$ & $0.20 \mathrm{ug} / \mathrm{L}$ & $690 \mathrm{ug} / \mathrm{L}$ \\
\hline Manganese & $25.0 \mathrm{ug} / \mathrm{L}$ & $0.20 \mathrm{ug} / \mathrm{L}$ & Non specified \\
\hline Mercury & $0.3 \mathrm{ug} / \mathrm{L}$ & $0.10 \mathrm{ug} / \mathrm{L}$ & $150 \mathrm{ug} / \mathrm{L}$ \\
\hline Nickel & $1.3 \mathrm{ug} / \mathrm{L}$ & $0.50 \mathrm{ug} / \mathrm{L}$ & $3,980 \mathrm{ug} / \mathrm{L}$ \\
\hline Selenium & $2.3 \mathrm{ug} / \mathrm{L}$ & $1.10 \mathrm{ug} / \mathrm{L}$ & $820 \mathrm{ug} / \mathrm{L}$ \\
\hline Uranium & $9.1 \mathrm{ug} / \mathrm{L}$ & $0.30 \mathrm{ug} / \mathrm{L}$ & Non specified \\
\hline Vanadium & $26.8 \mathrm{mg} / \mathrm{L}$ & $0.40 \mathrm{ug} / \mathrm{L}$ & $4,300 \mathrm{ug} / \mathrm{L}$ \\
\hline Zinc & $32.1 \mathrm{mg} / \mathrm{L}$ & $0.30 \mathrm{ug} / \mathrm{L}$ & $2,610 \mathrm{ug} / \mathrm{L}$ \\
\hline \multicolumn{4}{|l|}{ General Chemistry } \\
\hline Fluoride & $0.9 \mathrm{mg} / \mathrm{L}$ & $0.01 \mathrm{mg} / \mathrm{L}$ & $35 \mathrm{mg} / \mathrm{L}$ \\
\hline Chloride & $6.5 \mathrm{mg} / \mathrm{L}$ & $0.64 \mathrm{mg} / \mathrm{L}$ & Non specified \\
\hline Nitrate & $4.0 \mathrm{mg} / \mathrm{L}$ & $0.28 \mathrm{mg} / \mathrm{L}$ & Non specified \\
\hline Phosphate & $0.3 \mathrm{mg} / \mathrm{L}$ & $0.06 \mathrm{mg} / \mathrm{L}$ & Non specified \\
\hline Sulfate & $280 \mathrm{mg} / \mathrm{L}$ & $3.57 \mathrm{mg} / \mathrm{L}$ & Non specified \\
\hline Cyanides & Not Analyzed & --- & \begin{tabular}{|l|}
$1,200 \mathrm{ug} / \mathrm{L}$ (Total) \\
$860 \mathrm{ug} / \mathrm{L}$ (Amenable) \\
\end{tabular} \\
\hline Sulfide & Not Analyzed & $-\cdots$ & $14 \mathrm{mg} / \mathrm{L}$ \\
\hline $\begin{array}{l}\mathrm{LDR}=\text { land dispo } \\
\mathrm{MDL}=\text { method de } \\
\mathrm{mg} / \mathrm{L}=\text { milligrams } \\
\mathrm{ug} / \mathrm{L}=\text { microgram }\end{array}$ & $\begin{array}{l}\text { sal restriction. } \\
\text { tection limits. } \\
\text { per liter. } \\
\text { s per liter. }\end{array}$ & & \\
\hline
\end{tabular}


Table 2-3. Identification of Potential Leachate Constituents.

\begin{tabular}{|c|c|c|c|c|c|c|c|c|c|c|c|c|}
\hline \multirow[b]{2}{*}{ CAS \# } & \multirow[b]{2}{*}{ Common name } & \multicolumn{7}{|c|}{ Constituents } & \multirow[b]{2}{*}{$\begin{array}{c}\text { Consolidated } \\
\text { CAS\# list }\end{array}$} & \multirow[b]{2}{*}{$\begin{array}{c}\text { Not applicable by } \\
\text { professional } \\
\text { judgment }\end{array}$} & \multirow[b]{2}{*}{$\begin{array}{l}\text { Treatment } \\
\text { standard }\end{array}$} & \multirow[b]{2}{*}{$\begin{array}{c}\text { Potential } \\
\text { leachate } \\
\text { constituent }\end{array}$} \\
\hline & & $\underset{\bar{\equiv}}{\overline{\frac{0}{a}}}$ & 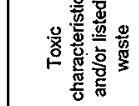 & 总 & 5 & $\begin{array}{l}\frac{\text { 葶 }}{\mathbf{w}} \\
\frac{\mathbf{g}}{8}\end{array}$ & 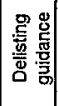 & $\stackrel{i g}{0+}$ & & & & \\
\hline $100-01-6$ & p-Nitroaniline & $x$ & P077 & $\mathrm{x}$ & $x$ & & & & $100-01-6$ & & Conc & 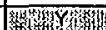 \\
\hline $100-02-7$ & p-Nitrophenol & $x$ & 0170 & $x$ & & & & & $100-02-7$ & & Conc & 7. \\
\hline$\overline{100-41-4}$ & Ethylbenzene & & F001-5 & $x$ & $x$ & $\mathrm{x}$ & $\bar{x}$ & & $100-41-4$ & & Cone & 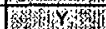 \\
\hline $100-42-5$ & Styrene & & & & & $x$ & $\mathrm{x}$ & & $100-42-5$ & & not specified & frocirima \\
\hline $100-51-6$ & Benzyl alcohol & $x$ & & & & $\bar{x}$ & & & $100-51-6$ & & not specified & 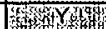 \\
\hline $10061-01-5$ & cis-1,3-Dichloropropylene & & F024 & $\bar{x}$ & $\bar{x}$ & & & & $10061-01-5$ & & $\begin{array}{l}\text { CMBST or } \\
\text { Cone }\end{array}$ & 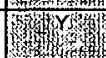 \\
\hline $10061-02-6$ & trans-1,3-Dichloropropylene & & F024 & $x$ & $\mathrm{x}$ & & & & $10061-02-6$ & & $\begin{array}{l}\text { CMBST or } \\
\text { Conc }\end{array}$ & (6) \\
\hline $100-75-4$ & N-Nitrosopiperidine & $x$ & U179 & $\mathrm{x}$ & $\mathrm{x}$ & $\bar{x}$ & & & $100-75-4$ & & Cone & Xer \\
\hline $10102-45-1$ & Thallium(l) nitrate (Thallium) & $x$ & $\mathrm{U} 217$ & & & & & & $10102-45-1$ & & STABL & 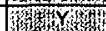 \\
\hline $101-14-4$ & 4,4'-Methylenebis(2-chloroaniline) & $x$ & $\overline{\mathbf{U 1 5 8}}$ & $x$ & $x$ & & & & $101-14-4$ & & Conc & 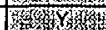 \\
\hline $101-27-9$ & Barban & $x$ & U280 & & $\vec{x}$ & & & & $101-27-9$ & & Conc & 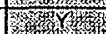 \\
\hline 101-55-3 & 4-Bromophenyl phenyl ether & $\bar{x}$ & 0030 & $\bar{x}$ & $\mathrm{x}$ & & & & $101-55-3$ & & Conc & 3. \\
\hline $1024-57-3$ & Heptachlor epoxide & $x$ & 0031 & $x$ & $x$ & $x$ & & & $1024-57-3$ & & Cono & 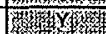 \\
\hline none & Heptachlor epoxide (alpha, beta, and gamma isomers) & $\bar{x}$ & & & & & & & $1024-57-3$ & & not specified. & 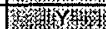 \\
\hline $1031-07-8$ & Endosulfan sulfate & & & $\mathrm{X}$ & $x$ & & & & $1031-07-8$ & & Conc & 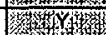 \\
\hline $105-67-9$ & 2,4-Dimethyiphenol & $\mathrm{x}$ & U101 & $\mathrm{x}$ & $\mathrm{x}$ & $\mathrm{x}$ & & & $105.67-9$ & & Conc & 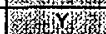 \\
\hline $10595-95-6$ & N-Nitrosomethylethylamine & $\mathrm{x}$ & & $\mathrm{x}$ & $x$ & $x$ & & & $10595-95-6$ & & Conc & Y \\
\hline $10605-21 \cdot 7$ & Carbendazim & $x$ & 0372 & & $x$ & & & & $10605-21-7$ & & Conc & 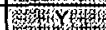 \\
\hline $106-445$ & p-Cresol & & D026, F001-5 & $\bar{x}$ & $x$ & & & & $106-44-5$ & & Conc & ${ }^{3}$ \\
\hline $106-46-7$ & p-Dichlorobenzene [1,4-Dichorobenzene] & $\mathrm{x}$ & 0027, U072 & $\mathrm{x}$ & $\mathrm{x}$ & $\mathrm{x}$ & & & $106-46-7$ & ? & Conc & 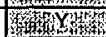 \\
\hline $25321-22-6$ & Dichlorobenzene, N.O.S. & $\mathrm{x}$ & & & & & & $\mathrm{x}$ & $106-46-7$ & & not specified & 9 \\
\hline $106-47-8$ & p-Chloroaniline & $\mathrm{x}$ & P024 & $x$ & $x$ & $\bar{x}$ & & & $106-47-8$ & & Conc & 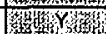 \\
\hline $106-50-3$ & p-Phenylenediamine & & & & & $\mathrm{x}$ & & & $106-50-3$ & & not specified & W \\
\hline 106-93-4 & Ethylene dibromide & $\mathrm{x}$ & U067 & $x$ & $x$ & $\mathrm{x}$ & & & $\longdiv { 1 0 6 - 9 3 - 4 }$ & & Conc & Why \\
\hline 107-05-1 & 3-Chloropropylene [Allyl chloride] & & F024 & $x$ & $x$ & $\bar{x}$ & & & $107-05-1$ & & $\begin{array}{l}\text { CMBST or } \\
\text { Conc }\end{array}$ & (5) \\
\hline $107-06-2$ & Ethylene dichloride [1,2-Dichloroethane] & $\mathrm{x}$ & 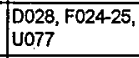 & $\bar{x}$ & $\bar{x}$ & $x$ & & & $107-06-2$ & & $\begin{array}{l}\text { CMBST or } \\
\text { Conc }\end{array}$ & $y^{4}$ \\
\hline 107-13-1 & Acryionitrile & $\bar{x}$ & U009 & $x$ & $x$ & $x$ & & & 107-13-1 & & Conc & $w_{\mathrm{X}}$ \\
\hline $108-05-4$ & Vinyl acetate & & & & & $\bar{x}$ & & & $108-05-4$ & & not specified & 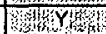 \\
\hline
\end{tabular}


Table 2-3. Identification of Potential Leachate Constituents.

\begin{tabular}{|c|c|c|c|c|c|c|c|c|c|c|c|c|c|}
\hline \multirow[b]{2}{*}{ CAS\# } & \multirow[b]{2}{*}{ Common name } & \multicolumn{7}{|c|}{ Constituents } & \multirow[b]{2}{*}{$\begin{array}{c}\text { Consolidated } \\
\text { CAS\# list } \\
\ldots \ldots \ldots\end{array}$} & \multirow[b]{2}{*}{$\begin{array}{c}\text { Not applicable by } \\
\text { professional } \\
\text { judgment } \\
\ldots \ldots\end{array}$} & \multirow[b]{2}{*}{$\begin{array}{l}\text { Treatment } \\
\text { standard }\end{array}$} & \multirow[b]{2}{*}{$\begin{array}{c}\begin{array}{c}\text { Potential } \\
\text { leachate } \\
\text { constituent }\end{array} \\
\ldots .\end{array}$} & \\
\hline & & 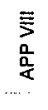 & 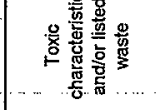 & 总 & $\frac{2}{5}$ & 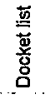 & 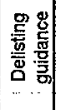 & $\begin{array}{l}\dot{d} \\
\dot{0} \\
z\end{array}$ & & & & & \\
\hline $108-10-1$ & Methyl isobutyl ketone [4-Methy-2-pentanone] & & F001-5, U161 & $\mathrm{x}$ & $\mathrm{x}$ & $\bar{x}$ & & & $108-10-1$ & & Conc & 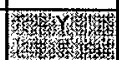 & \\
\hline $108-39-4$ & m-Cresol & & D024, F001-5 & $x$ & $x$ & & & & $108-39-4$ & & Conc & 3. & \\
\hline $108-60-1$ & Dichloroisopropyl ether & $x$ & U027 & & & $\mathrm{x}$ & & & $108-60-1$ & & Cone & 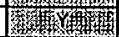 & \\
\hline $108-88-3$ & Toluene & $x$ & $\begin{array}{l}F 001-5, \text { U052, } \\
\text { U220 }\end{array}$ & $x$ & $x$ & $\mathrm{x}$ & & & $108-88-3$ & & Cone & \begin{tabular}{|c|c|}
$y_{3}$ \\
3
\end{tabular} & \\
\hline $108-90-7$ & Chlorobenzene & $x$ & $\begin{array}{l}\text { D021, F001-5, } \\
\text { U037 }\end{array}$ & $\mathrm{x}$ & $\mathrm{x}$ & $\mathrm{x}$ & & & $108-90-7$ & & Conc & ffoction & \\
\hline none & Chiorinated benzenes, N.O.S. & $x$ & & & & & & $x$ & $108-90-7$ & & not specified & M & \\
\hline $108-94-1$ & Cyciohexanone & & F001-5, U057 & $\mathrm{x}$ & $x$ & & & & $108-94-1$ & & $\begin{array}{l}\text { CMBST, Cone } \\
\text { or TCLP }\end{array}$ & 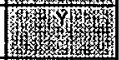 & \\
\hline $108-95-2$ & Phenol & $\mathrm{x}$ & U188 & $x$ & $x$ & $\mathrm{x}$ & & & $108-95-2$ & & Conc & 36y & \\
\hline none & HxCDFs [Hexachlorodibenzofurans] & $\bar{x}$ & F022, F026-28 & $\mathrm{x}$ & $x$ & & & & $109-99-9$ & & Conc & WX & \\
\hline none & PeCDFs [Pentachlorodibenzofurans] & $x$ & F022, F026-28 & $\mathrm{x}$ & $\bar{x}$ & & & & $109-99-9$ & & Conc & 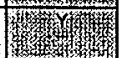 & \\
\hline none & TCDFs [Tetrachlorodibenzofurans] & $\mathrm{x}$ & F022, F026-28 & $\bar{x}$ & $x$ & & & & 109-99-9 & & Conc & 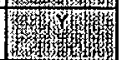 & \\
\hline none & HeCDFs [Heptachlorodibenzofurans] & $\mathrm{x}$ & & & & & & & $109-99-9$ & & Conc & 3. & \\
\hline $110-86-1$ & Pyridine & $\mathrm{x}$ & $\begin{array}{l}\text { D038, F001-5, } \\
\mathrm{U} 196\end{array}$ & $\bar{x}$ & $\mathrm{x}$ & $\bar{x}$ & & & $110-86-1$ & & Cone & 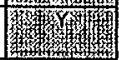 & \\
\hline $111-444$ & bis(2-Chloroethyl)ether [Dichloroethyl ether] & $\mathrm{x}$ & U025 & $\mathrm{x}$ & $\mathrm{x}$ & $\bar{x}$ & & & $111-44-4$ & & Conc & 3) & \\
\hline none & Chloroalkyl ethers, N.O.S. & $x$ & & & & & & $x$ & $11 .-44-4$ & & Conc & 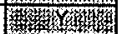 & \\
\hline $1114-71-2$ & Pebulate & $x$ & & & $x$ & & & & $1114-71-2$ & & Conc & 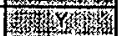 & \\
\hline $111-91-1$ & $\begin{array}{l}\text { bis(2-Chloroethoxy)methane } \\
\text { [Dichloromethoxy ethane] }\end{array}$ & $x$ & U024 & $x$ & $x$ & & & & $111-91-1$ & & Conc & 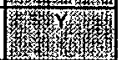 & \\
\hline $114-26-1$ & Propoxur & $\mathrm{x}$ & u411 & & $\bar{x}$ & & & & $114-26-1$ & & Cone & 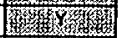 & $\sqrt{10}$ \\
\hline 115-29-7 & Endosulfan & $x$ & P050 & & & $x$ & & & $115-29-7$ & & Conc & yin & 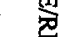 \\
\hline $117-81-7$ & Bis(2-Ethythexyl) phthalate & $x$ & $F 024, U 028$ & $x$ & $\bar{x}$ & $x$ & & & $117-81-7$ & & $\begin{array}{l}\text { CMBST or } \\
\text { Conc }\end{array}$ & 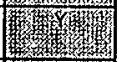 & \\
\hline $117-84-0$ & Di-n-octylphthalate & $x$ & 3107 & $x$ & $\mathrm{x}$ & $\bar{x}$ & & & $117-84-0$ & & Conc & 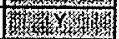 & న్స \\
\hline $118-79-6$ & 2,4,6-Tribromophenol & $\mathrm{x}$ & U408 & & & & & & $118-79-6$ & & Conc & 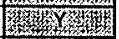 & - \\
\hline $120-12-7$ & Anthracene & & & $x$ & $x$ & $x$ & & & $120-12 \cdot 7$ & & Conc & Th- & $5 ?$ \\
\hline $12039-52-0$ & Thallium selenite (Selenium) & $x$ & P114 & & & & & & $12039-52-0$ & & TCLP & YYXX & 0 \\
\hline
\end{tabular}


DOE/RL-98-62, Rev. 0

$11 / 98$

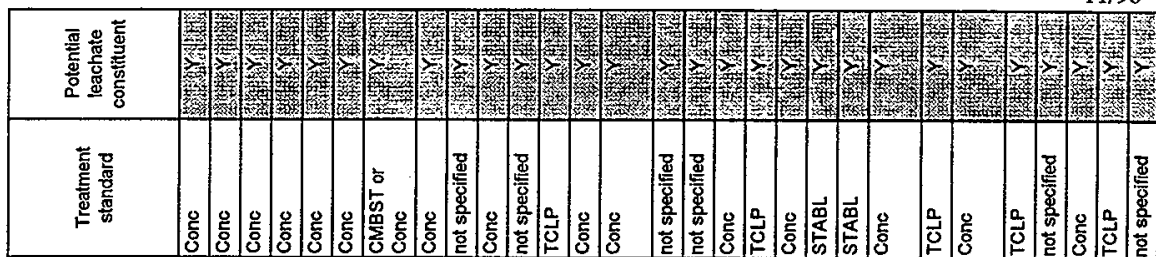

\section{ลे

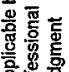

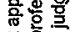 \\ sa}

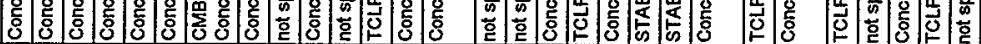

嘈

(5)

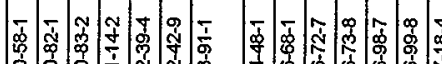

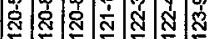

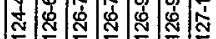

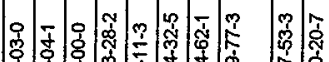

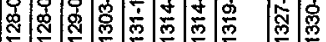

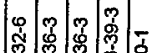

3.:

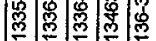

\section{S'O'N}

əouep!n6

6u!ns|jəa

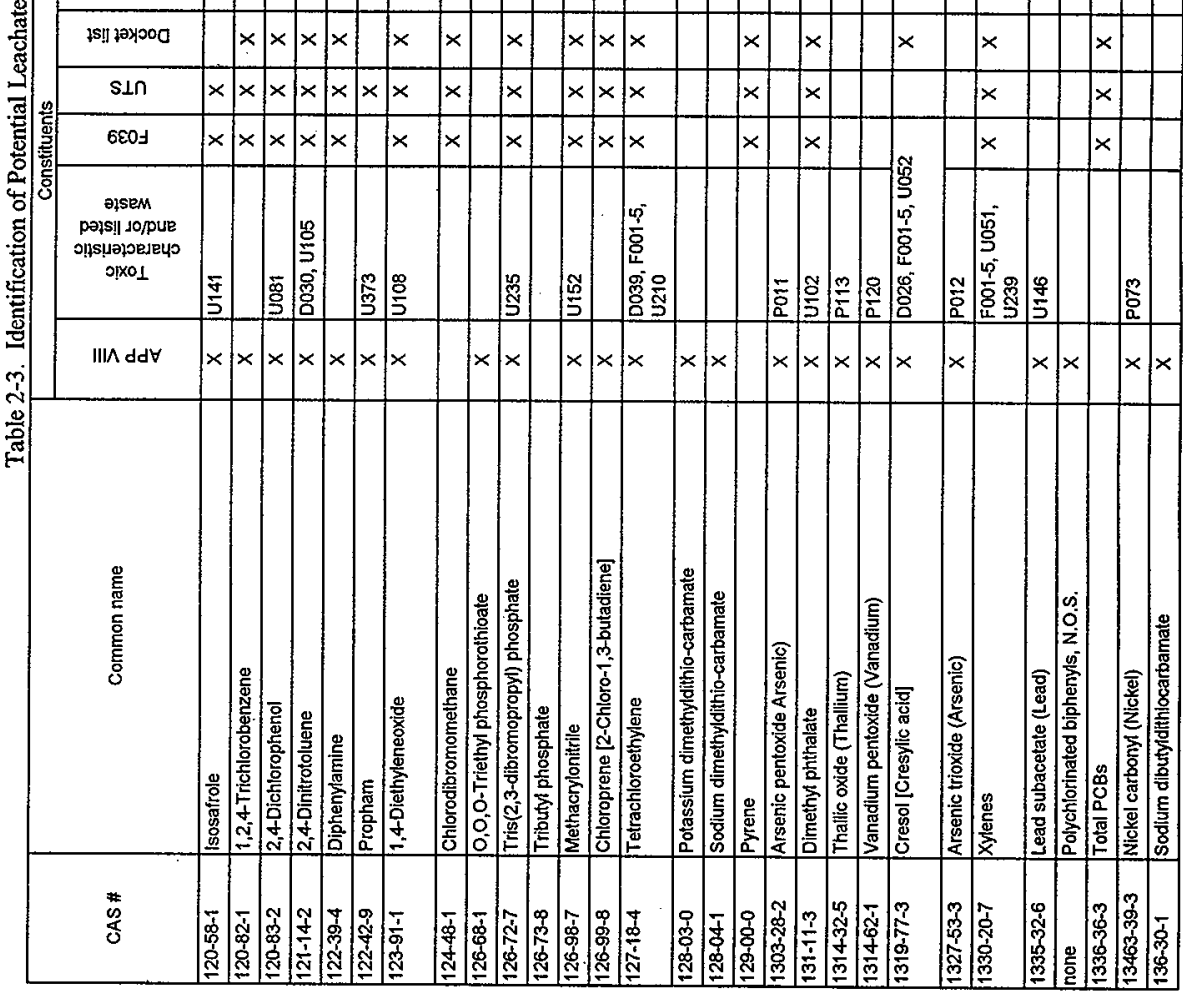


Table 2-3. Identification of Potential Leachate Constituents.

\begin{tabular}{|c|c|c|c|c|c|c|c|c|c|c|c|c|}
\hline \multirow[b]{2}{*}{ CAS \# } & \multirow[b]{2}{*}{ Common name. } & \multicolumn{7}{|c|}{ Constituents } & \multirow[b]{2}{*}{$\begin{array}{c}\text { Consolidated } \\
\text { CAS\# list }\end{array}$} & \multirow[b]{2}{*}{$\begin{array}{c}\begin{array}{c}\text { Not applicable by } \\
\text { professional } \\
\text { judgment }\end{array} \\
\end{array}$} & \multirow[b]{2}{*}{$\begin{array}{l}\text { Treatment } \\
\text { standard }\end{array}$} & \multirow[b]{2}{*}{$\begin{array}{c}\begin{array}{c}\text { Potentlal } \\
\text { leachate } \\
\text { constituent }\end{array} \\
. . . . . . . . . . .\end{array}$} \\
\hline & & 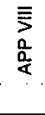 & 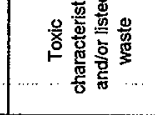 & $\begin{array}{c}\text { 量 } \\
\text { i. } \\
\cdots . . .\end{array}$ & $\stackrel{\infty}{5}$ & 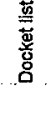 & 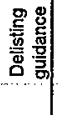 & $\begin{array}{l}\dot{0} \\
\dot{0} \\
\dot{z}\end{array}$ & & & & \\
\hline $137-29-1$ & Copper dimethyldithlocarbamate & $x$ & & & & & & & $137 \cdot 29 \cdot 1$ & & not specified & 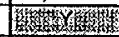 \\
\hline $137-30-4$ & Ziram & $\mathrm{x}$ & $\mathrm{P} 205$ & & $x$ & & & & $137-30-4$ & & Conc & 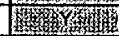 \\
\hline $137-41-7$ & Potassium n-methyldithio-carbamate & $x$ & & & & & & & $137-41-7$ & & not specified & 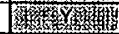 \\
\hline $137-42-8$ & Metam Sodium & $\mathrm{x}$ & & & & & & & $137-42-8$ & & not specified & 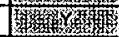 \\
\hline $13765-19-0$ & Calcium chromate (Chromium) & $x$ & 0032 & & & & & & $13765-19-0$ & & TCLP & 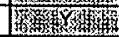 \\
\hline $140-57-8$ & Aramite & $x$ & : & $\mathrm{x}$ & $\mathrm{x}$ & & & & $140-57-8$ & & Conc & 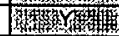 \\
\hline $141-78-6$ & Ethyl acetate & & F001-5, U112 & $\mathrm{x}$ & $x$ & $\mathrm{x}$ & & & $141-78-6$ & & Conc & 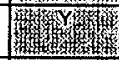 \\
\hline $14324-55-1$ & Ethyl Ziram & $x$ & & & & & & & 14324-55-1 & & not specified & 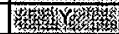 \\
\hline 143-33-9 & Sodium cyanide (Cyanides) & $\mathrm{x}$ & $\mathrm{P} 106$ & & & & & & 143-33-9 & & Cone & 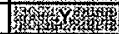 \\
\hline $143-50-0$ & Kepone & $x$ & U142 & $\mathrm{x}$ & $\mathrm{x}$ & $\mathrm{x}$ & & & $143-50-0$ & & Conc & 1) \\
\hline 144-34-3 & Selenium, tetrakis (dimethyl-dithiocarbamate) & $\mathrm{x}$ & & & & & & & $144.34-3$ & & TCLP & 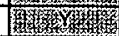 \\
\hline $14484-64-1$ & Ferbam & $\mathrm{x}$ & & & & & & & $14484-641$ & & not specified & 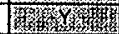 \\
\hline $148-18-5$ & Sodium diethyldithiocarbamate & $x$ & & & & & & & $148-18-5$ & & not specified & 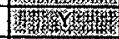 \\
\hline $151-50-8$ & Potassium cyanide (Gyanides) & $\mathrm{x}$ & PO98 & & & & & & $151-50-8$ & & Conc & 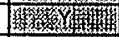 \\
\hline $15339-36-3$ & Manganese dimethyldithio-carbamate & $\mathrm{x}$ & P196 & & & & & & $15339-36-3$ & & Conc & 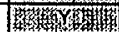 \\
\hline $1563-38-8$ & Carbofuran phenol & $x$ & 0367 & & $x$ & & & & $1563-38-8$ & & Conc & 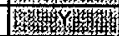 \\
\hline $1563-66-2$ & Carbofuran & $x$ & P127 & & $x$ & & & & $1563-66-2$ & & Cone & 13 \\
\hline $156-59-2$ & cis-1,2-Dichloroethylene & & & & & $\mathrm{x}$ & & & $156-59-2$ & & not specified & 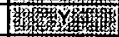 \\
\hline $156-60-5$ & trans-1,2-Dichloroethylene & $\mathrm{x}$ & U079 & $\bar{x}$ & $x$ & $x$ & & & $156-60-5$ & & Conc & 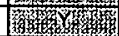 \\
\hline $1634-02-2$ & Tetrabutylthiuram disulfide & $\mathrm{x}$ & & & & & & & $1634-02-2$ & & not specified & 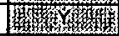 \\
\hline $1646-88-4$ & Aldicarb'sulfone & $\mathrm{x}$ & P203 & & $x$ & & & & $1646-88-4$ & & Cone & 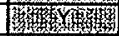 \\
\hline $16984-48-8$ & Fluoride & & & $x$ & $x$ & $\bar{x}$ & & & $16984-48-8$ & & Conc & 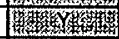 \\
\hline $17804-35-2$ & Benomy & $x$ & U271 & & $x$ & & & & $17804-35-2$ & & Conc & 16) \\
\hline $18496-25-8$ & Sulfide & & & $x$ & $x$ & & $x$ & & $18496-25-8$ & & Conc & 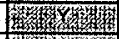 \\
\hline $1888-71-7$ & Hexachloropropene & $x$ & U243 & $x$ & $x$ & & & & $1888-71-7$ & & Cone & 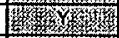 \\
\hline 189-64-0 & Dibenzo[ $[\mathrm{a}, \mathrm{h}] \mathrm{pyrene}$ & $x$ & & & & & & & $189-64-0$ & & not specified & W \\
\hline $191-242$ & Benzo[g,h,i]perylene & & & $x$ & $x$ & & & & 191-24-2 & & Cone & 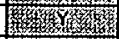 \\
\hline $192-65-4$ & Dibenzo $[a, e] p y r e n e$ & $x$ & & $x$ & $x$ & & & & $192-65-4$ & & Conc & 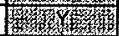 \\
\hline $1929-77-7$ & Vernolate & $x$ & & & $\mathrm{x}$ & & & & $1929-77 \cdot 7$ & & Conc & 3 \\
\hline 193-39-5 & Indeno[1,2,3-cd]pyrene & $\mathrm{x}$ & U137 & $x$ & $x$ & $\mathrm{x}$ & & & $193-39-5$ & & Conc & Yig \\
\hline $2008-41-5$ & Butylate & $x$ & & & $x$ & & & & $2008-41-5$ & & Conc & 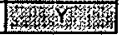 \\
\hline
\end{tabular}


Table 2-3. Identification of Potential Leachate Constituents.

\begin{tabular}{|c|c|c|c|c|c|c|c|c|c|c|c|c|}
\hline \multirow[b]{2}{*}{ CAS\# } & \multirow[b]{2}{*}{ Common name } & \multicolumn{7}{|c|}{ Constituents } & \multirow[b]{2}{*}{$\begin{array}{c}\text { Consolidated } \\
\text { CAS\# list }\end{array}$} & \multirow[b]{2}{*}{$\begin{array}{c}\text { Not applicable by } \\
\text { professional } \\
\text { judgment }\end{array}$} & \multirow[b]{2}{*}{$\begin{array}{l}\text { Treatment } \\
\text { standard }\end{array}$} & \multirow[b]{2}{*}{$\begin{array}{c}\text { Potential } \\
\text { leachate } \\
\text { constituent }\end{array}$} \\
\hline & & $\begin{array}{l}\text { 紊 } \\
\frac{0}{2} \\
\frac{0}{\alpha}\end{array}$ & 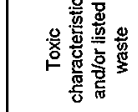 & 总 & $\stackrel{2}{5}$ & $\begin{array}{l}\frac{\underline{\underline{m}}}{\bar{m}} \\
\frac{\overrightarrow{8}}{8}\end{array}$ & 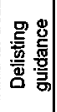 & $\begin{array}{l}n \\
0 \\
z\end{array}$ & & & & \\
\hline $2032-65-7$ & Methiocarb & $x$ & P199 & & $\mathrm{x}$ & & & & $2032-65-7$ & & Conc & 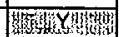 \\
\hline $205-82-3$ & Benzofiffluoranthene & $x$ & & & & & & & $205-82-3$ & & not specified & 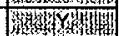 \\
\hline 205-99-2 & Benzo[b]fluoranthene & $\mathrm{x}$ & & $x$ & $\mathrm{x}$ & $x$ & & & 205-99-2 & & Conc & 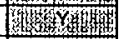 \\
\hline $206-440$ & Filuoranthene & $\mathrm{x}$ & U120 & $\mathrm{x}$ & $x$ & $\mathrm{x}$ & & & $206-440$ & & Conc & 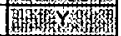 \\
\hline $207-08-9$ & Benzo(k)fluoranthene & $x$ & & $x$ & $x$ & & & & $207-08-9$ & & Cone & 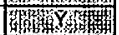 \\
\hline $20816-12-0$ & Osmium tetroxide & $x$ & P087 & & & & & & $20816-12-0$ & & $\begin{array}{l}\text { RTHRM (alt. } \\
\text { STABL) }\end{array}$ & $\mathrm{H}^{4}$ \\
\hline $208-96-8$ & Acenaphthylene & & & $\mathrm{x}$ & $x$ & & & & $208-96-8$ & & Conc & 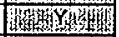 \\
\hline $218-01.9$ & Chrysene & $x$ & U050 & $\mathrm{x}$ & $x$ & $\mathrm{x}$ & & & $218-01 \cdot 9$ & & Conc & Shy \\
\hline $2242-67-1$ & Molinate & $\mathrm{x}$ & & & $\mathrm{x}$ & & & & $2212-67-1$ & & Conc & 5y \\
\hline $224-42-0$ & Dibenz[a,j]acridine & $\mathrm{x}$ & & & & & & & 224-42-0 & & not specified & 3y \\
\hline $226-36-8$ & Dibenz[a, h]acridine & $\mathrm{x}$ & & & & & & & $226-36-8$ & & not specified & 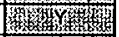 \\
\hline $22781-23-3$ & Bendiocarb & $x$ & U278 & & $x$ & & & & $22781-23-3$ & & Conc & Fi \\
\hline $2303-17-5$ & Triallate & $x$ & 4389 & & $x$ & & & & $2303-17-5$ & & Cone & 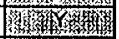 \\
\hline $23135-22-0$ & oxamyl & $x$ & P194 & & $x$ & & & & $23135-22-0$ & & Conc & 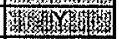 \\
\hline $23564-05-8$ & Thiophanate-methyl & $\mathrm{x}$ & U409 & & $x$ & & & & $23564-05-8$ & & Cone & (7) \\
\hline $23950-58-5$ & Pronamide & $x$ & U192 & $x$ & $\bar{x}$ & $x$ & & & $23950-58-5$ & & Conc & 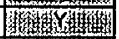 \\
\hline $2631-37-0$ & Promecarb & $x$ & $\mathrm{P} 201$ & & $\mathrm{x}$ & & & & $2631-37-0$ & & Conc & 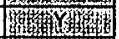 \\
\hline $26545-73-3$ & Dichloropropanol, N.O.S. & $\mathrm{x}$ & & & & & & $x$ & $26545-73-3$ & & not specified & 4 Y \\
\hline $298-00-0$ & Methyl parathion & $x$ & $\mathrm{P} 071$ & $x$ & $x$ & $\bar{x}$ & & & $298-00-0$ & & Conc & 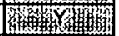 \\
\hline 298-02-2 & Phorate & $x$ & P094 & $x$ & $x$ & $x$ & & & 298-02-2 & & Conc & (7) \\
\hline $298-04-4$ & Disulfoton & $x$ & P039 & $x$ & $x$ & $x$ & & & $298-04-4$ & & Conc & 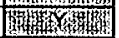 \\
\hline $301-04-2$ & Lead acetate (Lead) & $\mathrm{x}$ & U144 & & & & & & $301-04-2$ & & TCLP & 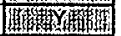 \\
\hline $30558-43-1$ & $A 2213$ & $x$ & 0394 & & $x$ & & & & $30558-43-1$ & & Conc & 14 \\
\hline $309-00-2$ & Aldrin & $x$ & $\mathrm{POO4}$ & $x$ & $x$ & $x$ & & & $309-00-2$ & & Conc & 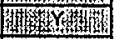 \\
\hline $315-18-4$ & Mexacarbate & $x$ & $\mathrm{P} 128$ & & $\mathrm{x}$ & & & & $315-18-4$ & & Conc & Uty \\
\hline $319-84-6$ & alpha-BHC & & $D 013, U_{129}$ & $x$ & $x$ & $\mathrm{x}$ & & & $319-84-6$ & & Cone & 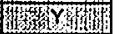 \\
\hline $319-85-7$ & beta-BHC & & $D 013, \cup 129$ & $x$ & $x$ & $x$ & & & 319-85-7 & & Conc & 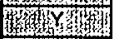 \\
\hline $319-86-8$ & delfa-8HC & & $D 013, \cup 129$ & $x$ & $x$ & & & & $319-86-8$ & & Conc & Y \\
\hline $33213-65-9$ & Endosulfan II & & & $x$ & $x$ & & & & $33213-6-5$ & & Cone & 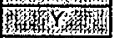 \\
\hline $3424-82-6$ & o,p'-DDE & & & $x$ & $x$ & & & & $3424-82-6$ & & Conc & 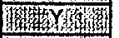 \\
\hline $39638-32-9$ & bis(Chlorolsopropy)ether & & & $\mathrm{x}$ & $x$ & & & & $39638-32-9$ & & Conc & $4 y_{4}$ \\
\hline
\end{tabular}


Table 2-3. Identification of Potential Leachate Constituents.

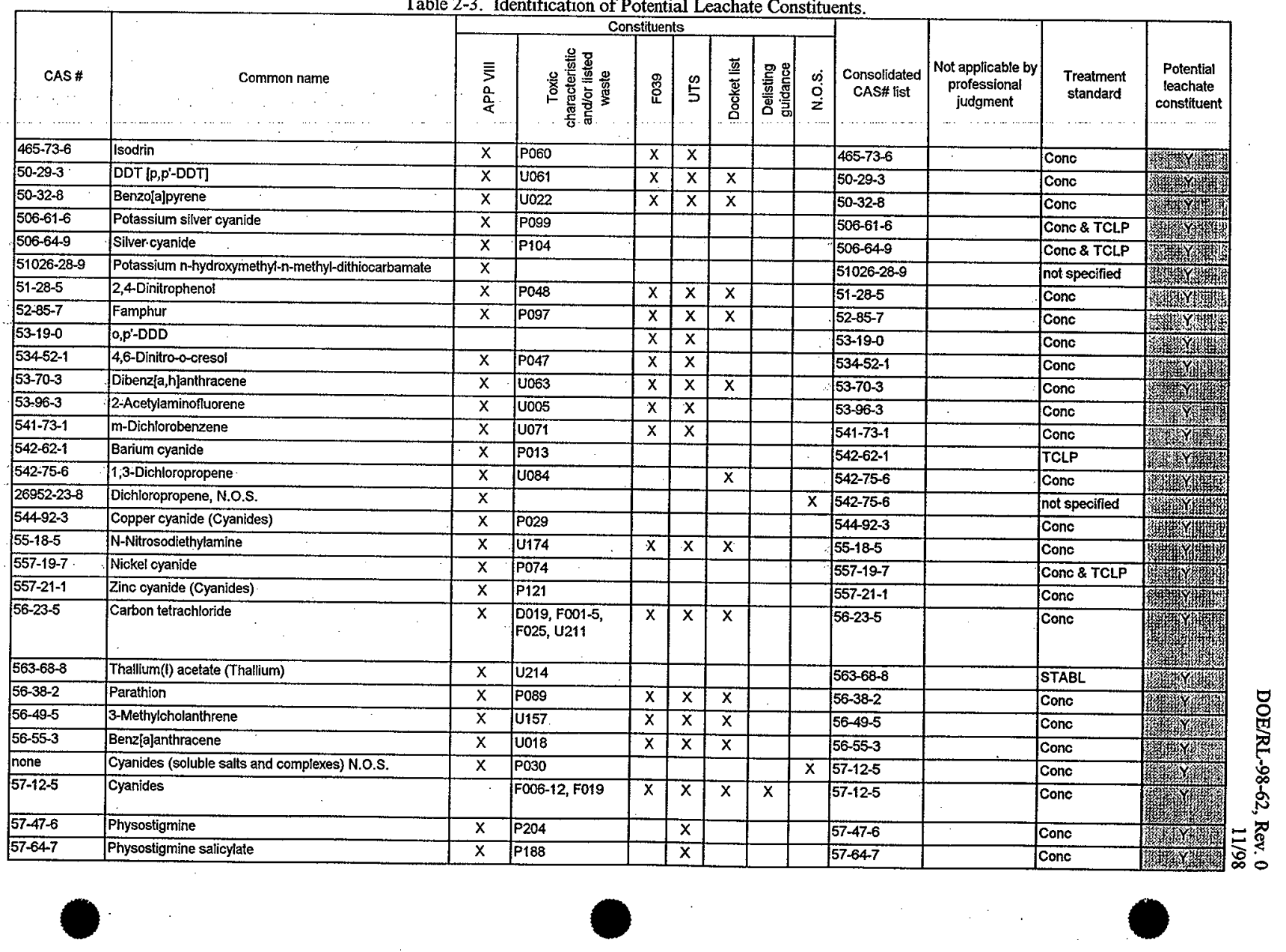


Table 2-3. Identification of Potential Leachate Constituents.

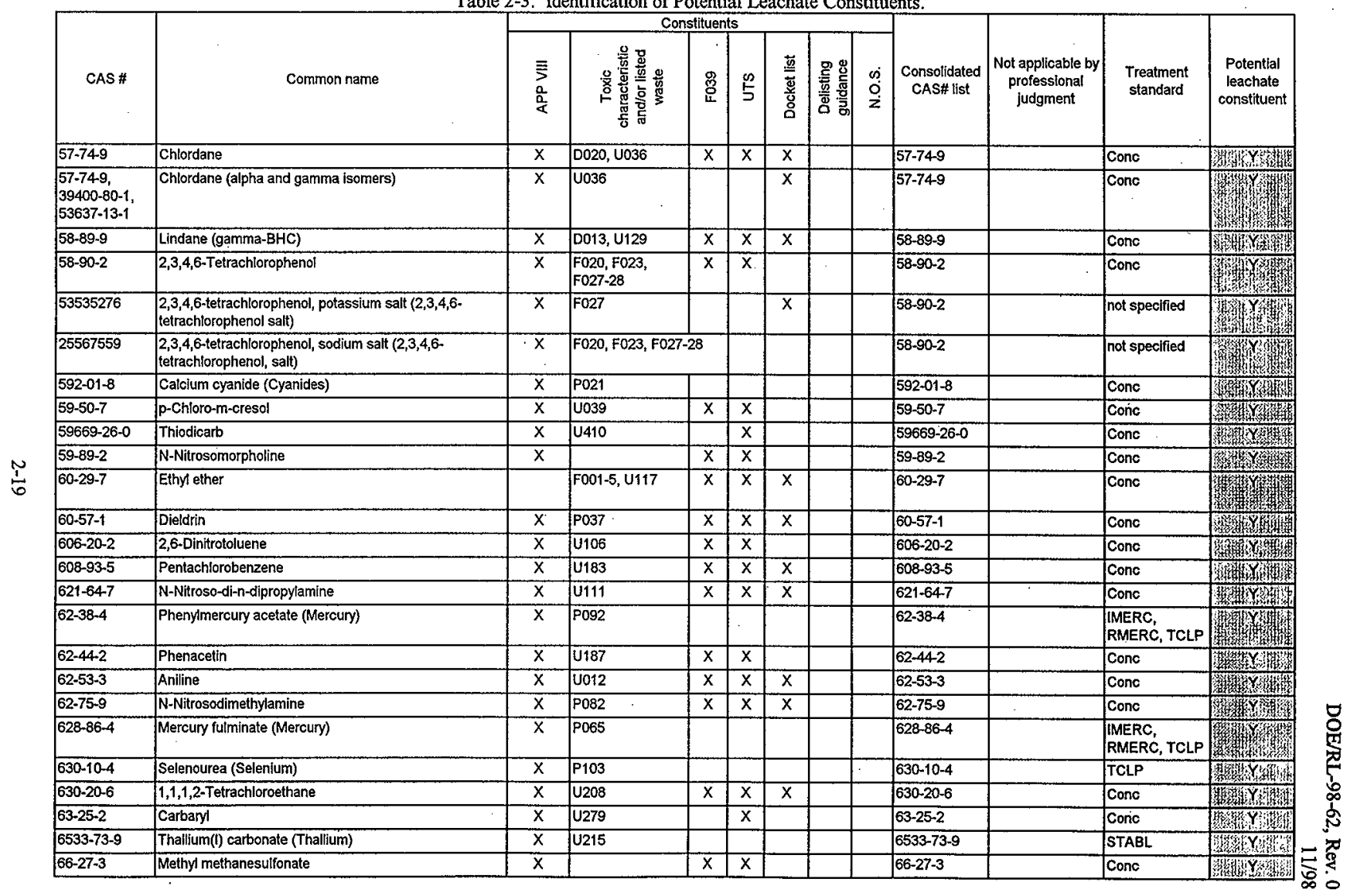




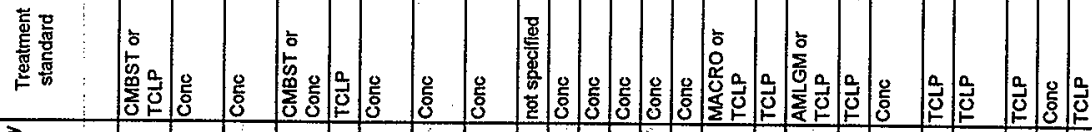

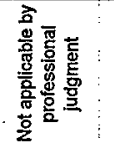

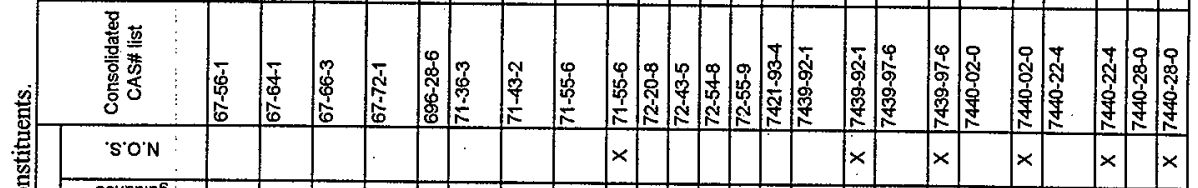

(1)

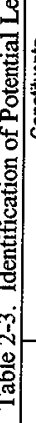

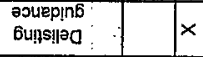

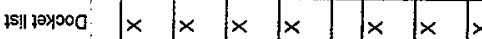

$\sin :|x| x|x| x|c| x|x| x$

$6 \varepsilon 01: \quad x \quad x|x|$

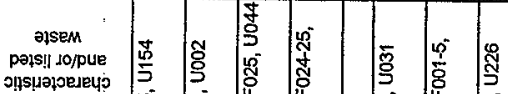

opxo1

送

$\operatorname{lindd\forall }$

(2.
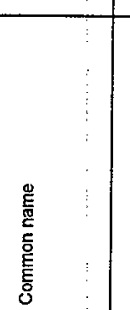

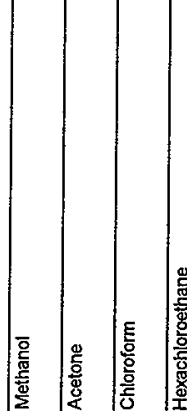

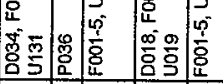

范

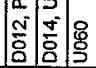

$\times \times \times \times \times x$

$+$

(

\begin{tabular}{|c|c|c|}
\hline & \\
\hline & & \\
\hline
\end{tabular}

$\times$

$x$

\begin{tabular}{|l|l|l|l|}
\hline$x$ & $x$ & $x$ & $x$ \\
\hline$x$ & $x$ & $x$ & $x$ \\
\hline
\end{tabular}


Table 2-3. Identification of Potential Leachate Constituents.

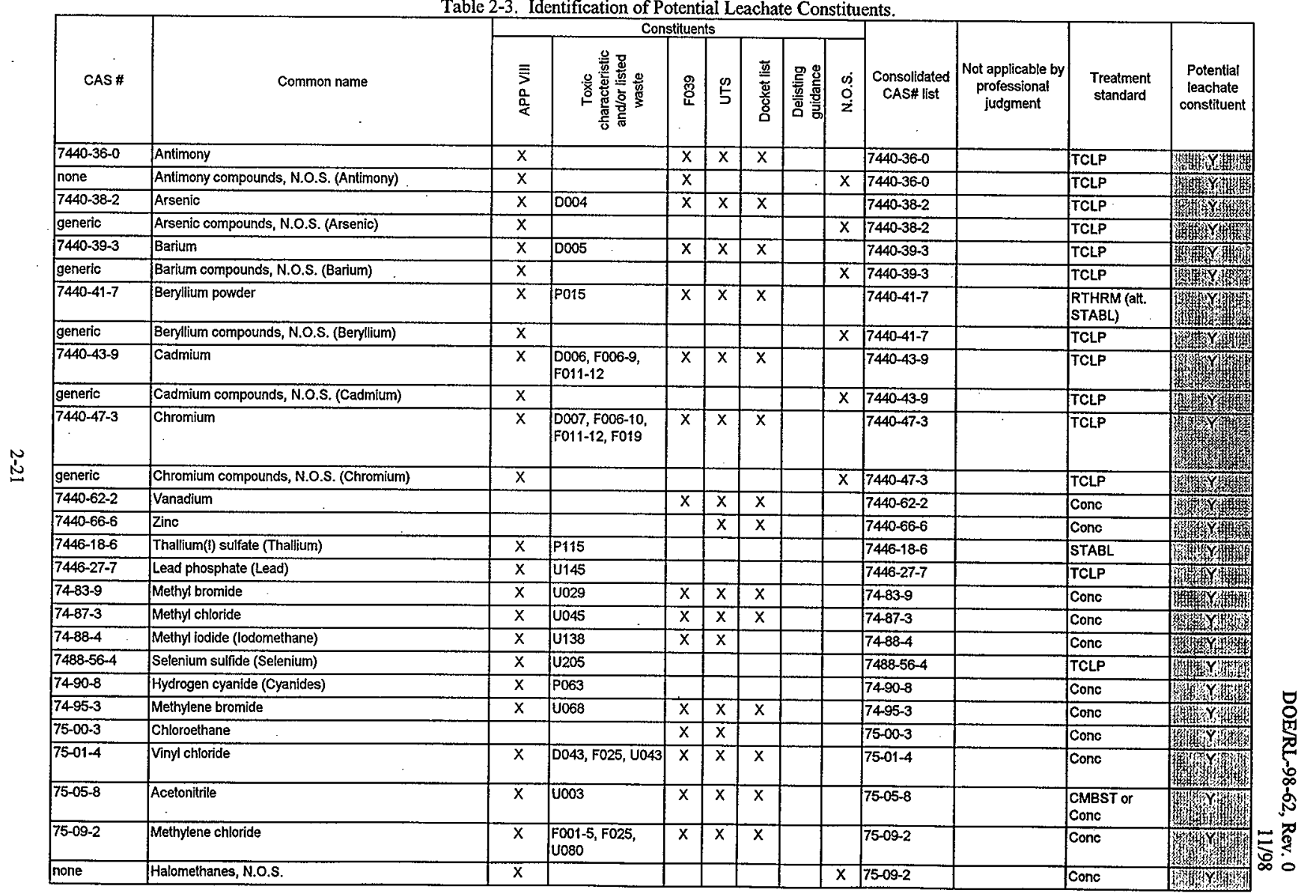


DOE/RL-98-62, Rev. 0

$11 / 98$

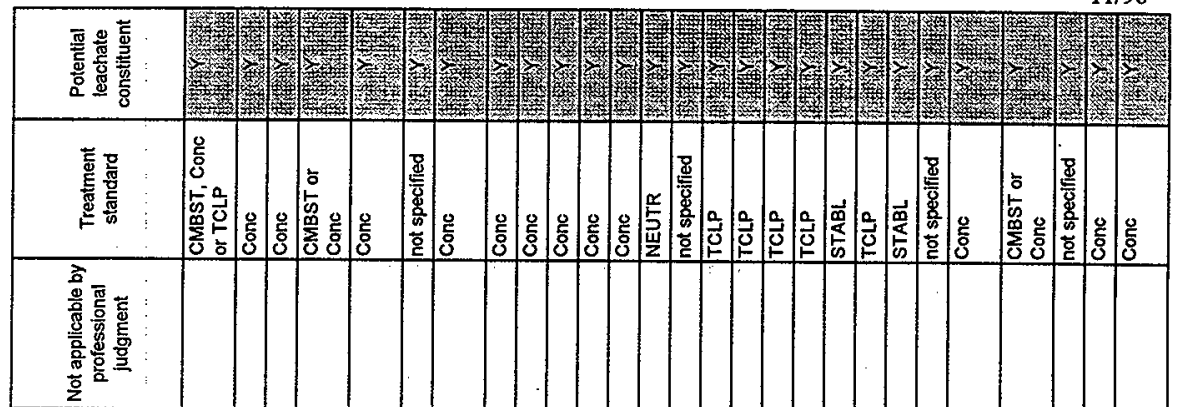

\begin{tabular}{|c|c|c|c|c|c|c|c|c|c|c|c|c|c|c|c|c|c|c|c|c|c|c|c|c|c|c|}
\hline \pm & 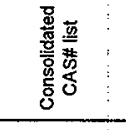 & $\frac{p}{0}$ & 就 & 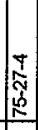 & 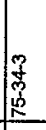 & 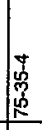 & 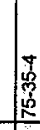 & 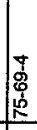 & 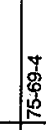 & 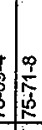 & 吉 & $\underset{\rho}{\stackrel{\rho}{\rho}}$ & \begin{tabular}{|l}
$\infty$ \\
\\
$\vdots$ \\
0
\end{tabular} & 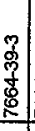 & 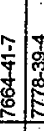 & ל. & 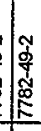 & 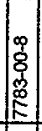 & $\mid \begin{array}{c}9 \\
\frac{1}{2} \\
\frac{1}{2} \\
\frac{2}{5} \\
\end{array}$ & 帘 & 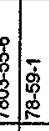 & 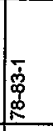 & 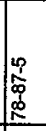 & 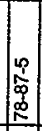 & 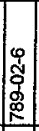 & 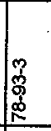 \\
\hline & SON & . & & & & & $x$ & & $\ddot{x}$ & & & & & & & & $x$ & & & & & & & $x$ & & \\
\hline & $\begin{array}{l}\text { exuepint } \\
\text { Gu!s!nao }\end{array}$ & & & & & & & & & & & & & & & & & & & & $x$ & & & & & \\
\hline & is! fayoog & $x$ & $x$ & $\times$ & $\times$ & $x$ & & $x$ & & $\times$ & & $x$ & $x$ & & & $x$ & & & & & $x$ & $x$ & $x$ & & & $x$ \\
\hline & $\sin$ & $x$ & $x$ & $\times$ & $x$ & $x$ & & $x$ & & $x$ & $x$ & $x$ & $\times$ & & & $x$ & & & & & & $x$ & $x$ & & $x$ & $x$ \\
\hline & $6 \varepsilon 0 \mathrm{~A}$ & $\times$ & $x$ & $\times$ & $x$ & $x$ & & $x$ & & $x$ & & $x$ & $x$ & & & $x$ & & & & & & $\times$ & $x$ & & $x$ & $x$ \\
\hline ثَ & 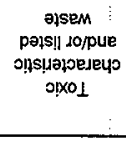 & స̃ & 工్ & & 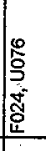 & 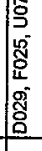 & & 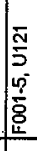 & & 㤏 & & 章 & 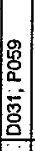 & $\stackrel{5}{5}$ & $\stackrel{0}{\circ}$ & 5 & & 诘 & & $\left.\frac{0}{i}\right) \stackrel{9}{\frac{1}{i}}$ & & \begin{tabular}{|l}
8 \\
5 \\
5 \\
40 \\
18 \\
58 \\
\end{tabular} & 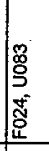 & & & 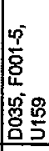 \\
\hline & \|\|$\wedge d d \forall$ & $x$ & $x$ & & $x$ & $x$ & $x$ & $x$ & $\times$ & $x$ & $x$ & & $\times$ & $x$ & $x$ & $x$ & $x$ & $x$ & $\times>$ & $\times x$ & & $x$ & $x$ & $x$ & & $x$ \\
\hline & 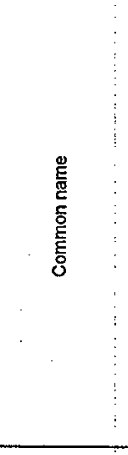 & 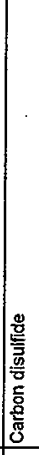 & 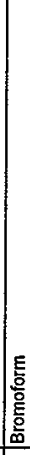 & 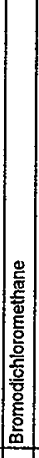 & 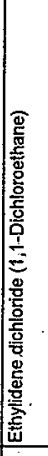 & 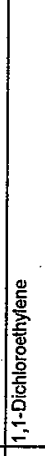 & 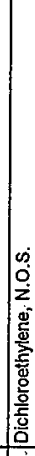 & 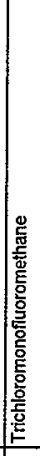 & 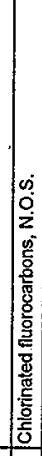 & 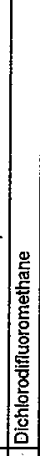 & 喜 & 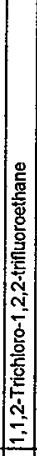 & 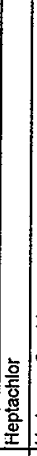 & 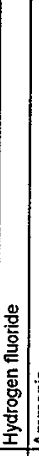 & 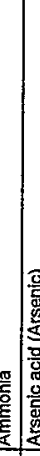 & 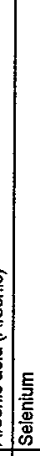 & 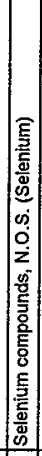 & 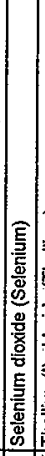 & 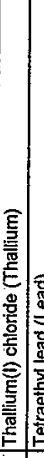 & 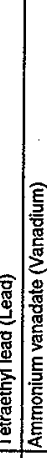 & 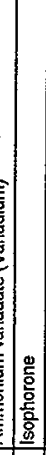 & 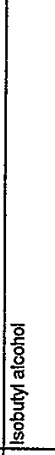 & 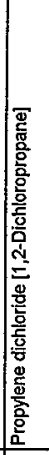 & 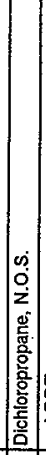 & 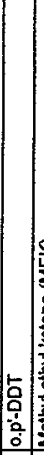 & 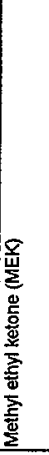 \\
\hline & ֻै & 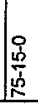 & 峛 & 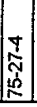 & 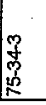 & 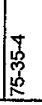 & 路 & & 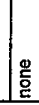 & $\mid \frac{\infty}{\Gamma}$ & 咅 & $\stackrel{\Phi}{\frac{\alpha}{\alpha}}$ & $\begin{array}{l}\text { 里 } \\
\vdots \\
\vdots \\
0\end{array}$ & 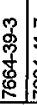 & $\begin{array}{c}\frac{1}{y} \\
\frac{y}{d}\end{array}$ & : & $\begin{array}{c}\mathscr{\Xi} \\
\stackrel{0}{\subseteq} \\
\end{array}$ & $\mid$ & 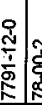 & 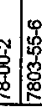 & $\frac{\sqrt{\infty}}{\Phi_{\infty}^{\infty}}$ & $\mid \begin{array}{l}\overline{1} \\
\vdots \\
0 \\
0\end{array}$ & 柋 & 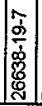 & 电 & శ్ \\
\hline
\end{tabular}




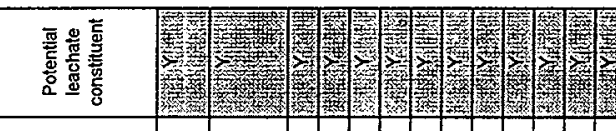

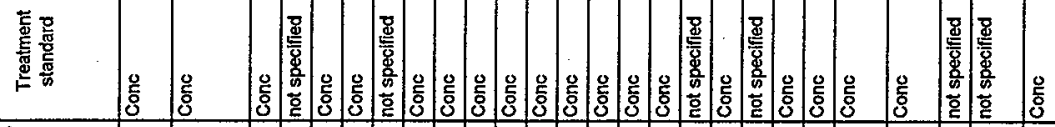

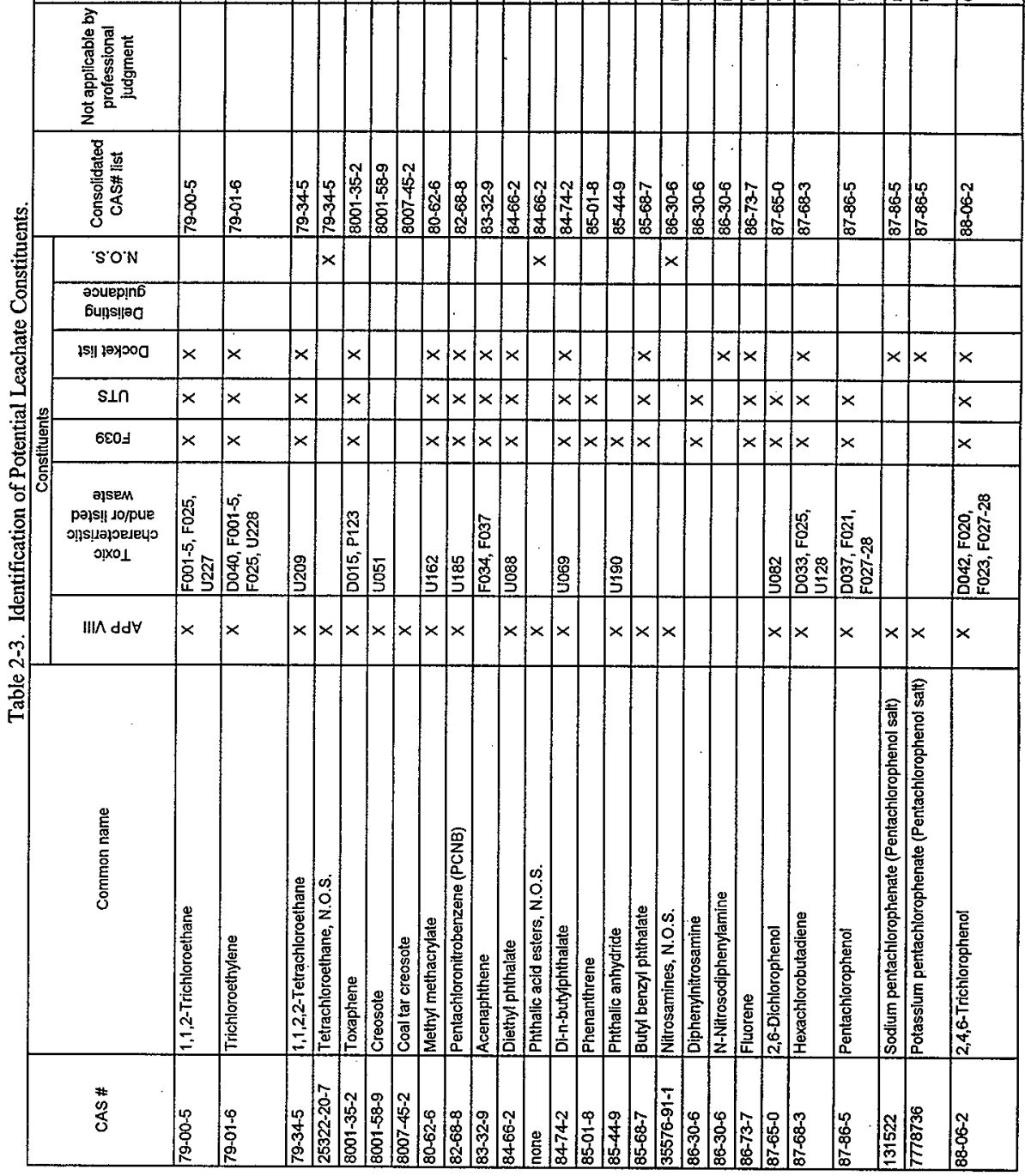


Table 2-3. Identification of Potential Leachate Constituents.

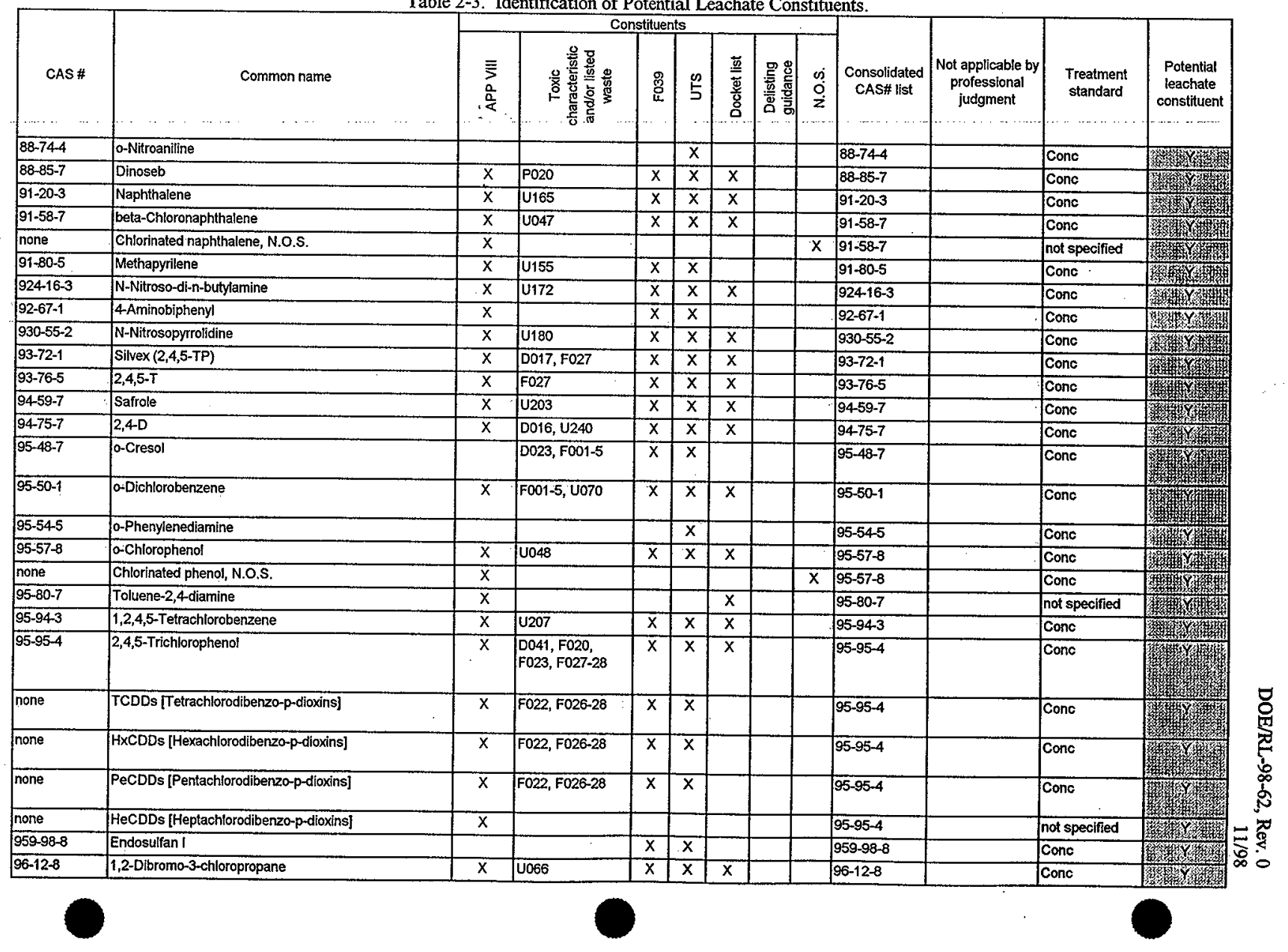


Table 2-3. Identification of Potential Leachate Constituents.

\begin{tabular}{|c|c|c|c|c|c|c|c|c|c|c|c|c|}
\hline \multirow[b]{2}{*}{ CAS\# } & \multirow[b]{2}{*}{ Common name } & \multicolumn{7}{|c|}{ Constituents } & \multirow[b]{2}{*}{$\begin{array}{c}\text { Consolidated } \\
\text { CAS\# list }\end{array}$} & \multirow[b]{2}{*}{$\begin{array}{c}\text { Not applicable by } \\
\text { professional } \\
\text { judgment }\end{array}$} & \multirow[b]{2}{*}{$\begin{array}{l}\text { Treatment } \\
\text { standard }\end{array}$} & \multirow[b]{2}{*}{$\begin{array}{c}\text { Potential } \\
\text { leachate } \\
\text { constituent }\end{array}$} \\
\hline & & $\begin{array}{l}\bar{\equiv} \\
\frac{a}{a} \\
k\end{array}$ & 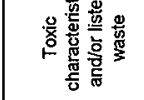 & g్ & $\stackrel{\infty}{5}$ & 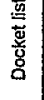 & 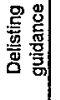 & $\begin{array}{l}\dot{j} \\
\dot{0} \\
\dot{z}\end{array}$ & & & & \\
\hline $96-18-4$ & 1,2,3-Trichloropropane & $x$ & & $x$ & $x$ & $\mathrm{x}$ & & & $96-18-4$ & & Conc & 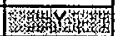 \\
\hline $25735-29-9$ & Trichloropropane, N.O.S. & $x$ & & & & & & $x$ & $96-18-4$ & & not specified & W \\
\hline 97.63 .2 & Ethyl methacrylate & $\bar{x}$ & U118 & $\mathrm{x}$ & $x$ & $\mathrm{x}$ & & & $97-63-2$ & & Conc & W \\
\hline $97-77-8$ & Disulfiram & $x$ & & & & & & & $97-77-8$ & & not specified & 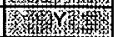 \\
\hline $98-05-5$ & Benzenearsonic acid & $x$ & & & & & & & $98-05-5$ & & not specified & 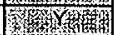 \\
\hline $98-86-2$ & Acetophenone & $\mathrm{x}$ & U004 & $x$ & $\mathrm{x}$ & $x$ & & & $98-86-2$ & & Cone & 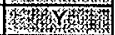 \\
\hline $98-95-3$ & Nitrobenzene & $\mathrm{x}$ & $\begin{array}{l}\text { D036, F001-5, } \\
\text { U169 }\end{array}$ & $\bar{x}$ & & $\bar{x}$ & & & $98-95-3$ & & Cone & ${ }_{4} x_{14}$ \\
\hline $99-55-8$ & 5-Nitro-0-toluidine & $x$ & U181 & $x$ & & & & & $99-55-8$ & & Conc & 致, \\
\hline $99-65-0$ & 1,3-Dinitrobenzene & & & & & $x$ & & & $99-65-0$ & & not specified & 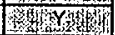 \\
\hline none & Oil \& Grease & & & & & & $x$ & & none & & not specified & W \\
\hline $100-25-4$ & 1,4-Dinitrobenzene & & & $\mathrm{x}$ & $\mathrm{x}$ & $x$ & & & $100-25-4$ & Dyestuff & Conc & $\mathrm{N}$ \\
\hline $100-44-7$ & Benzyl chloride & $\mathrm{X}$ & $\mathrm{PO} 28$ & & & $x$ & & & $100-447$ & & CMBST & $\mathrm{N}$ \\
\hline $10102-43-9$ & Nitric oxide & $\mathrm{X}$ & P076 & & & & & & $10102-43-9$ & & ADGAS & $N$ \\
\hline $10102-44-0$ & Nitrogen dioxide & $x$ & P078 & & & & & & $10102-44-0$ & & ADGAS & $N$ \\
\hline $103-85-5$ & Phenylthiourea & $x$ & P093 & & & & & & 103-85-5 & Pesticide & CMBST & $N$ \\
\hline $106-49-0$ & p-Toluidine & $x$ & U353 & & & $x$ & & & $106-49-0$ & & CMBST & $N$ \\
\hline $106-51-4$ & p-Benzoquinone & $x$ & U197 & & & & & & $106-51-4$ & & CMBST & $\mathrm{N}$ \\
\hline $106-89-8$ & Epichlorohydrin & $x$ & U041 & & & $\mathrm{x}$ & & & $106-89-8$ & & CMBST & $N$ \\
\hline $107-02-8$ & Acrolein & $\mathrm{X}$ & $\mathrm{P003}$ & $\mathrm{x}$ & $x$ & $\mathrm{x}$ & & & $107-02-8$ & & CMBST & $N$ \\
\hline $107-10-8$ & n-Propylamine & $x$ & U194 & & & & & & $107-10-8$ & \begin{tabular}{|c|} 
Pesticide, \\
Dyestuff, \\
Pharmaceutical, \\
Polymer \\
\end{tabular} & CMBST & $N$ \\
\hline 107-12-0 & Ethyl cyanide & $x$ & P101 & $x$ & $x$ & & & & $107-12-0$ & Solvent & Conc & $\mathrm{N}$ \\
\hline $107-18-6$ & Allyt alcohol & $x$ & P005 & & & & & & $107-18-6$ & Fungicide & CMBST & $N$ \\
\hline $107-19-7$ & Propargyl alcohol & $\mathrm{x}$ & $\mathrm{P} 102$ & & & & & & $107-19.7$ & & CMBST & $\mathbf{N}$ \\
\hline $107-20-0$ & Chloroacetaldehyde & $x$ & $\mathrm{P023}$ & & & & & & $107-20-0$ & & CMBST & $\mathrm{N}$ \\
\hline $107-30-2$ & Chloromethyl methyl ether & $x$ & U046 & & & & & & $107-30-2$ & & CMBST & $N$ \\
\hline $107-49-3$ & Telraethyl pyrophosphate & $x$ & $\mathrm{P} 111$ & & & & & & $107-49 \cdot 3$ & & CMBST & $N$ \\
\hline $108-31-6$ & Maleic anhydride & $x$ & U147 & & & & & & $108-31-6$ & & CMBST & $N$ \\
\hline $108-46-3$ & Resorcinol & $x$ & U201 & & & & & & $108-46-3$ & & CMBST & $\mathrm{N}$ \\
\hline $108-98-5$ & Thlophenol & $x$ & P014 & & & & & & $108-98-5$ & & CMBST & $\mathrm{N}$ \\
\hline
\end{tabular}


Table 2-3. Identification of Potential Leachate Constituents.

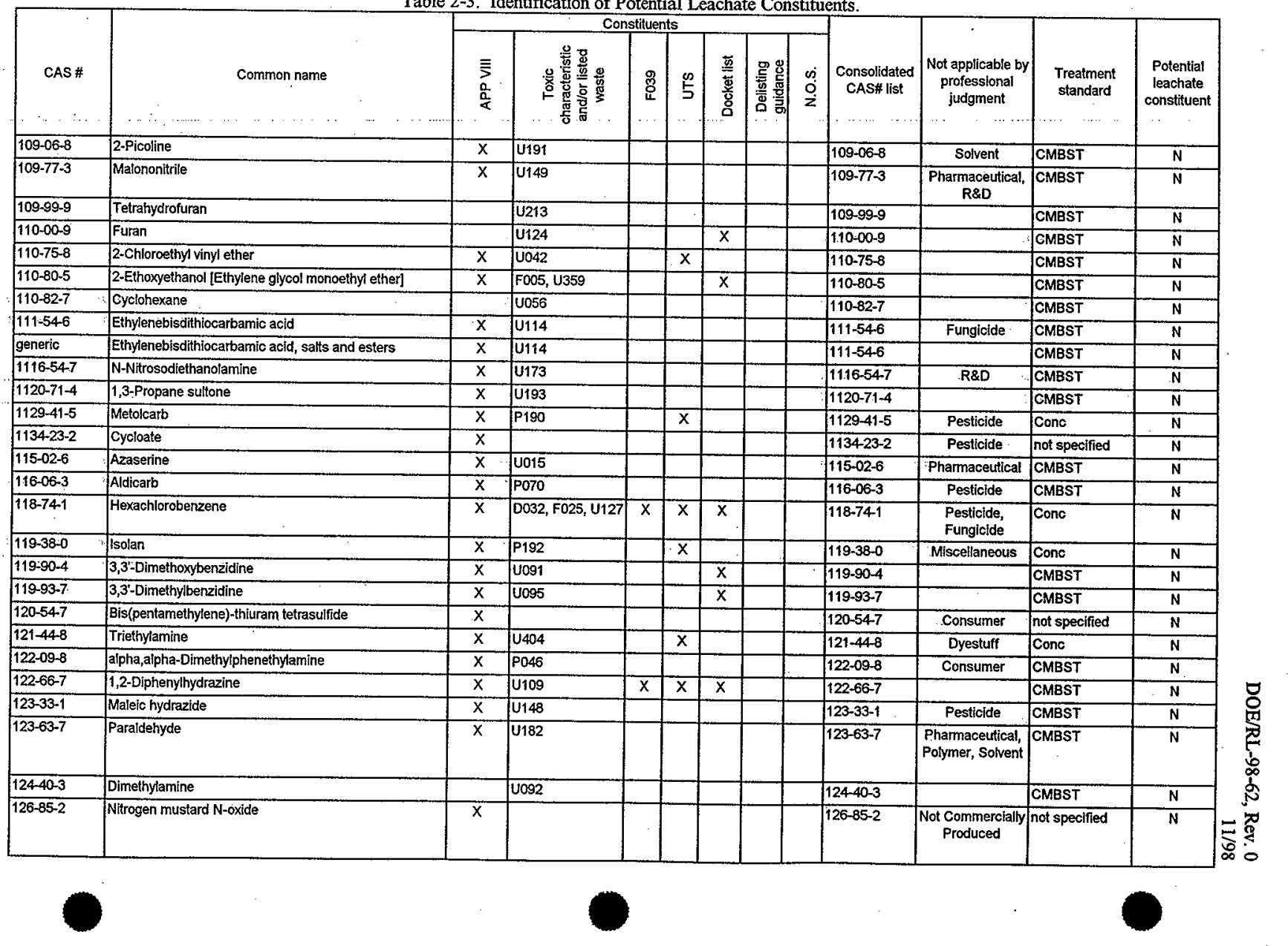


Table 2-3. Identification of Potential Leachate Constituents.

\begin{tabular}{|c|c|c|c|c|c|c|c|c|c|c|c|c|}
\hline \multirow[b]{2}{*}{ CAS\# } & \multirow[b]{2}{*}{ Common name } & \multicolumn{7}{|c|}{ Constikuents } & \multirow[b]{2}{*}{$\begin{array}{c}\text { Consolidated } \\
\text { CAS\# list }\end{array}$} & \multirow[b]{2}{*}{$\begin{array}{c}\text { Not applicable by } \\
\text { professional } \\
\text { judgment }\end{array}$} & \multirow[b]{2}{*}{$\begin{array}{l}\text { Treatment } \\
\text { standard }\end{array}$} & \multirow[b]{2}{*}{$\begin{array}{l}\text { Potential } \\
\text { leachate } \\
\text { constituent }\end{array}$} \\
\hline & & $\frac{5}{\frac{5}{a}}$ & 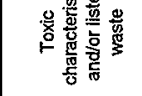 & 8 & $\stackrel{62}{5}$ & 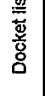 & 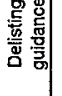 & $\begin{array}{l}\dot{v} \\
\dot{0} \\
z\end{array}$ & & & & \\
\hline $130-15-4$ & 1,4-Naphthoquinone & $\mathrm{x}$ & U166 & & & & & & $130-15-4$ & Polymer & CMBST & $N$ \\
\hline $1314-80-3$ & Phosphorus sulfide & & U189 & & & & & & $1314-80-3$ & & CMBST & $N$ \\
\hline $1314-84-7$ & Zinc phosphide & $\mathrm{x}$ & $\mathrm{P} 122, \mathrm{U} 249$ & & & & & & $1314-84-7$ & & CMBST & $N$ \\
\hline $131-74-8$ & Ammonium picrate & & P009 & & & & & & $131-74-8$ & & CMBST & $N$ \\
\hline 131-89-5 & 2-Cyclohexyl-4,6-dinitrophenol & $\mathrm{X}$ & P034 & & & & & . & $131-89-5$ & . & CMBST & $N$ \\
\hline $13256-22-9$ & N-Nitrososarcosine & $x$ & & & & & & & $13256-22-9$ & $\begin{array}{c}\text { Non- } \\
\text { Commercially } \\
\text { Produced }\end{array}$ & not specified & $N$ \\
\hline $1338-23-4$ & Methyl ethyl ketone peroxide & $x$ & U160 & & & & & & $1338-23-4$ & Polymer & CMBST & $N$ \\
\hline 134-32-7 & alpha-Naphthylamine & $\mathrm{x}$ & U167 & & & & & & $134-32-7$ & & CMBST & $\mathrm{N}$ \\
\hline $137-26-8$ & Thiram & $\mathrm{x}$ & $\mathrm{U} 244$ & & & & & & $137-26-8$ & & CMBST & $\bar{N}$ \\
\hline $1402-68-2$ & Allatoxins & $\mathrm{x}$ & & & & & & & $1402-68-2$ & R\&D & not specified & $\mathbf{N}$ \\
\hline $140-88-5$ & Ethyl acrylate & & U113 & & & & & & $140-88-5$ & & CMBST & $N$ \\
\hline $142-847$ & Dipropylamine & & U110 & & & & & & $142-84-7$ & & $\overline{C M B S T}$ & $N$ \\
\hline $145-73-3$ & Endothall & $x$ & P088 & & & $\mathrm{x}$ & & & $145-73-3$ & Pesticide & CMBST & $N$ \\
\hline $1464-53-5$ & 1,2:3,4-Diepoxybutane & $\mathrm{x}$ & U085 & & & & & & $1464-53-5$ & R\&D, Polymer & CMBST & $N$ \\
\hline $148-82-3$ & Melphalan [alanine nitrogen mustard] & $\mathrm{x}$ & U150 & & & & & & $148-82-3$ & Pharmaceutical & CMBST & $\bar{N}$ \\
\hline $14901-08-7$ & Cycasin & $\mathrm{x}$ & . & & & & & & $14901-08-7$ & $\begin{array}{c}\text { Pharmaceutical, } \\
\text { Not Commercially } \\
\text { Produced }\end{array}$ & not specified & $\bar{N}$ \\
\hline $151-56-4$ & Ethyleneimine & $x$ & P054 & & & & & & $151-56-4$ & & CMBST & $N$ \\
\hline $152-16-9$ & Octamethylpyrophosphoramide & $x$ & P085 & & & $x$ & & & $152-16-9$ & Pesticide & CMBST & $\mathrm{N}$ \\
\hline $1615-80-1$ & N,N'-Dlethylhydrazine & $x$ & U086 & & $\cdot$ & & & & $1615-80-1$ & & CMBST & $\mathbf{N}$ \\
\hline $16543-55-8$ & N-Nitrosonornicotine & $\mathrm{x}$ & & & & & & & $16543-55-8$ & Consumer & not specified & $N$ \\
\hline $16752-77-5$ & Methomyl & $\mathrm{x}$ & P066 & & $x$ & & & & $16752-77-5$ & & CMBST & $N$ \\
\hline 17702-57-7 & Formparanate & $x$ & P197 & & $x$ & & & & $17702-57-7$ & Pesticide & Conc & $N$ \\
\hline 18883-66-4 & Streptozotocin & $x$ & 4206 & & & & & & $18883-66-4$ & Pharmcetutical & CMBST & $N$ \\
\hline $189-55-9$ & Dibenzo[a,i]pyrene & $\mathrm{x}$ & U064 & & & & & & 189-55-9 & & CMBST & $N$ \\
\hline $194-59-2$ & 7H-Dibenzo[c,g]carbazole & $\mathrm{x}$ & & & & & & & $194-59-2$ & $\begin{array}{c}\text { Not Commercially } \\
\text { Produced }\end{array}$ & not specified & $\bar{N}$ \\
\hline 20830-81-3 & Daunomycin & $\bar{x}$ & U059 & & & & & & $20830-81-3$ & Pharmaceutica! & CMBST & $\mathbf{N}$ \\
\hline
\end{tabular}


Table 2-3. Identification of Potential Leachate Constituents.

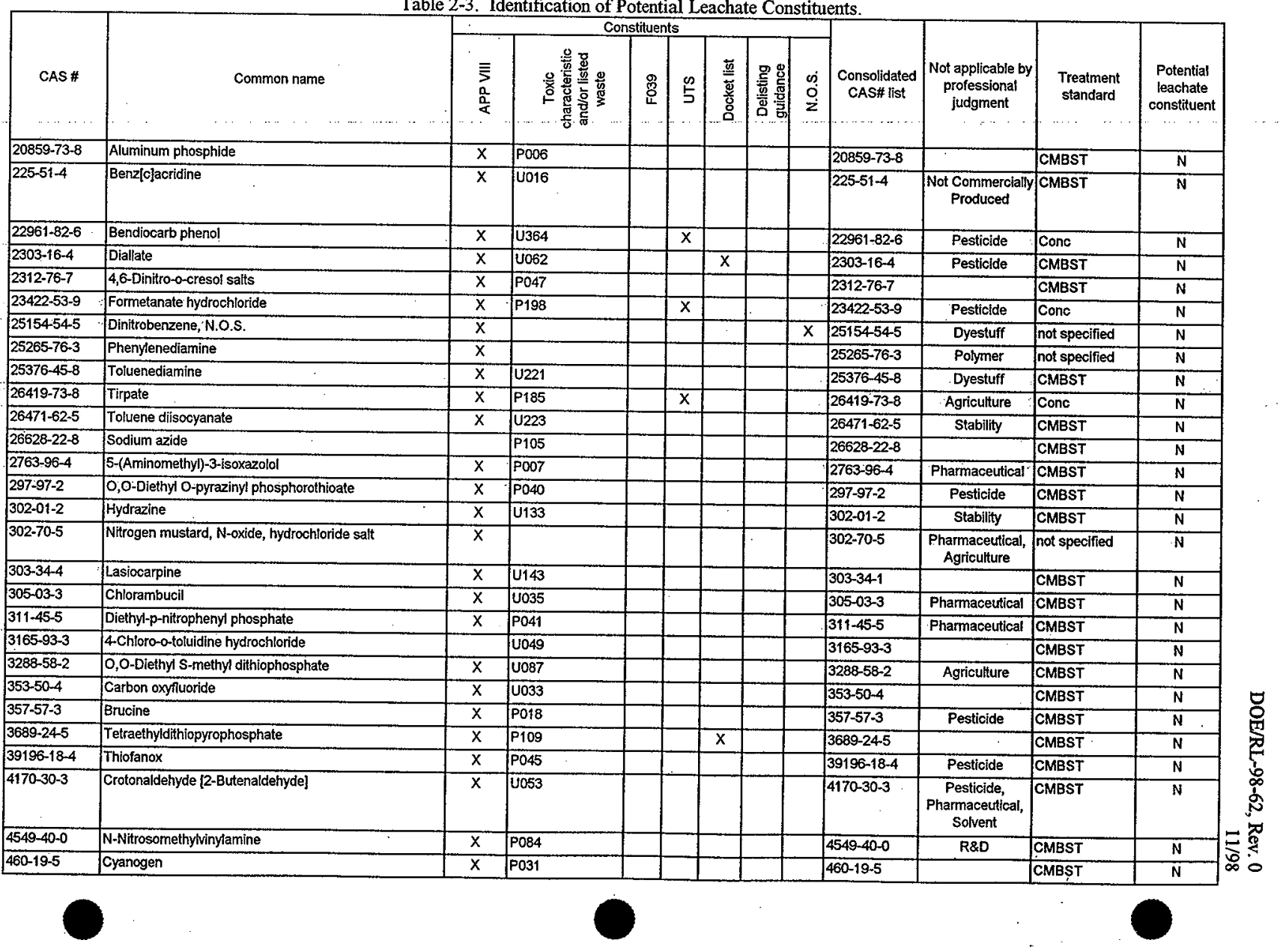


Table 2-3. Identification of Potential Leachate Constituents.

\begin{tabular}{|c|c|c|c|c|c|c|c|c|c|c|c|c|}
\hline \multirow[b]{2}{*}{ CAS\# } & \multirow[b]{2}{*}{ Common name } & \multirow[b]{2}{*}{ · } & \multicolumn{6}{|c|}{ Constituents } & \multirow[b]{2}{*}{$\begin{array}{l}\text { Consolidated } \\
\text { CAS\# list }\end{array}$} & \multirow[b]{2}{*}{$\begin{array}{c}\text { Not applicable by } \\
\text { professional } \\
\text { judgment }\end{array}$} & \multirow[b]{2}{*}{$\begin{array}{l}\text { Treatment } \\
\text { standard }\end{array}$} & \multirow[b]{2}{*}{$\begin{array}{l}\text { Potential } \\
\text { leachate } \\
\text { constituent }\end{array}$} \\
\hline & & & 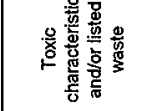 & 量 & $\stackrel{0}{5}$ & 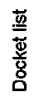 & 总 & $\begin{array}{l}\dot{0} \\
0 \\
z\end{array}$ & & & & \\
\hline $492-80-8$ & Auramine & $x$ & So14 & & & & & & $492-80-8$ & Dyestuff & CMBST & $N$ \\
\hline 494-03-1 & Chlornaphazin & $x$ & $\mathrm{U} 026$ & & & & & & 49403-1 & Pharmaceutical & CMBST & $N$ \\
\hline $496-72-0$ & Toluene-3,4-diamine & $\bar{x}$ & & & & & & & $496-72-0$ & $\begin{array}{c}\text { Dyestuff, } \\
\text { Consumer, Misc. }\end{array}$ & not specified & $\mathrm{N}$ \\
\hline $50-00-0$ & Formaldehyde & $\mathrm{X}$ & U122 & & & & & & $50-00-0$ & . & CMBST & $N$ \\
\hline $50-07-7$ & Mitomycin C & $x$ & 4010 & & & & & & $50-07-7$ & Pharmaceutical & CMBST & $N$ \\
\hline $50-18-0$ & Cyclophosphamide & $\mathrm{X}$ & U058 & & & & & & $50-18-0$ & Pharmaceutical & CMBST & $N$ \\
\hline $504-24-5$ & 4-Aminopyridine & $x$ & $\mathrm{PO08}$ & & & & & & $504-24-5$ & Pharmaceutical & CMBST & $N$ \\
\hline $504-60-9$ & 1,3-Pentadiene & & U186 & & & & & & $504-60-9$ & & CMBST & $N$ \\
\hline 50-55-5 & Reserpine & $\mathrm{X}$ & U200 & & & & & & $50-55-5$ & Pharmaceutical & CMBST & $N$ \\
\hline $505-60-2$ & Mustard gas & $x$ & & & & & & & $505-60-2$ & Military & not specified & $N$ \\
\hline $506-68-3$ & Cyanogen bromide & $\mathrm{X}$ & U246 & & & & & & $506-68-3$ & & CMBST & $N$ \\
\hline $506-77-4$ & Cyanogen chloride & $x$ & P033 & & & $x$ & & & $506-77-4$ & & CMBST & $N$ \\
\hline $509-14-8$ & Tetranitromethane & $x$ & $\mathrm{P} 112$ & & & & & & $509-14-8$ & & CMBST & $N$ \\
\hline $510-15 \cdot 6$ & Chlorobenzilate & $x$ & U038 & $\bar{x}$ & $\mathrm{x}$ & $x$ & & & $510-15-6$ & & CMBST & $N$ \\
\hline $51-43-4$ & Epinephrine & $x$ & P042 & & & & & & $51-43-4$ & Pharmaceutical & CMBST & $N$ \\
\hline $51-52-5$ & Propythiouracil & $x$ & & & & & & & $51-52-5$ & Pharmaceutical & not specifled & $\mathrm{N}$ \\
\hline $51-75-2$ & Nitrogen mustard & $x$ & & & & & & & $51-75-2$ & Military & not speclfied & $\mathrm{N}$ \\
\hline $51-79-6$ & Ethyl carbamate (urethane) & $x$ & U238 & & & & & & $51-79-6$ & & CMBST & $\mathrm{N}$ \\
\hline $52-24-4$ & Tris(1-aziridinyt)phosphine sulfide & $x$ & & & & & & & $52-24-4$ & Pharmaceutical & not specified & $\mathbf{N}$ \\
\hline $52888-80-9$ & Prosulfocarb & $x$ & U387 & & $\mathrm{x}$ & & & & $52888-80-9$ & Agriculture & Conc & $\mathbf{N}$ \\
\hline $533-74-4$ & Dazomet & $x$ & & & & & & & $533-74-4$ & $\begin{array}{l}\text { Pesticide, } \\
\text { Agriculture }\end{array}$ & not specified & $\mathbf{N}$ \\
\hline $5344-82-1$ & 1-(o-Chlorophenyl)thiourea & $x$ & P026 & & & & & & $5344-82-1$ & Pesticide & CMBST & $\mathrm{N}$ \\
\hline $540-73-8$ & 1,2-Dimethylhydrazine & $x$ & Uog9 & & & & & & $540-73-8$ & & CMBST & $N$ \\
\hline $54-11-5$ & Nicotine & $x$ & $P 075$ & & & & & & $54-11-5$ & & CMBST & $N$ \\
\hline generic & Nicotine salts & $x$ & P075 & & & . & & & $54-11-5$ & & CMBST & $\mathbf{N}$ \\
\hline $541-53-7$ & Dithiobiuret & $\bar{x}$ & PO49 & & & & & & $541-53-7$ & Pesticide & CMBST & $N$ \\
\hline $542-76-7$ & 3-Chloropropionitrile & $\bar{x}$ & $\mathrm{P} 027$ & & & & & & $542-76-7$ & \begin{tabular}{|c|}
$\begin{array}{c}\text { Pharmaceutical, } \\
\text { Polymer }\end{array}$ \\
\end{tabular} & CMBST & $\mathrm{N}$ \\
\hline $542-88-1$ & \begin{tabular}{|l|} 
Dichloromethyl ether \\
\end{tabular} & $x$ & P016 & & & & & & $542-88-1$ & & CMBST & $N$ \\
\hline $55285-14-8$ & Carbosulfan & $x$ & P189 & & $x$ & & & & $55285-148$ & Agriculture & Conc & $N$ \\
\hline
\end{tabular}


Table 2-3. Identification of Potential Leachate Constituents.

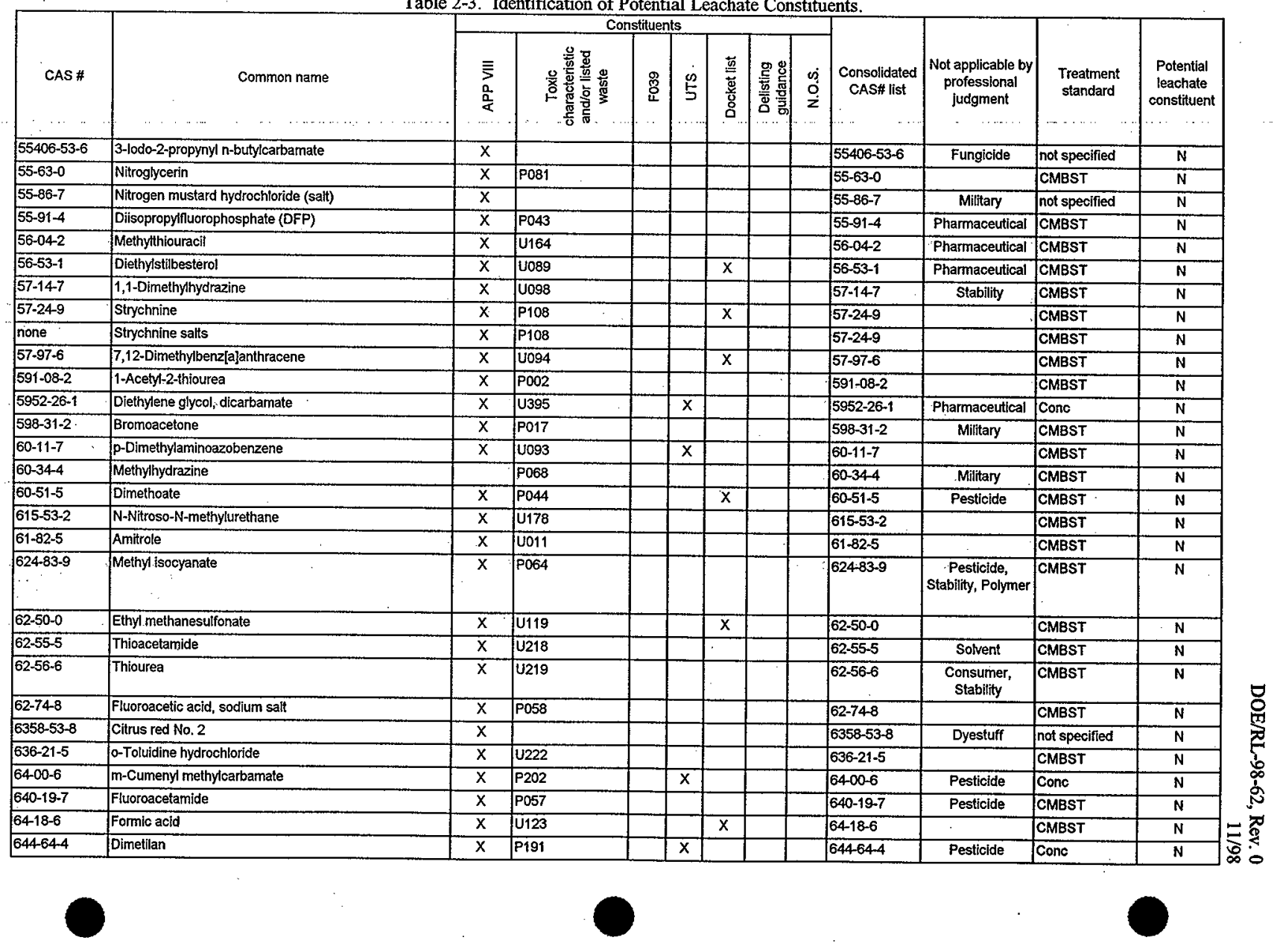


Table 2-3. Identification of Potential Leachate Constituents.

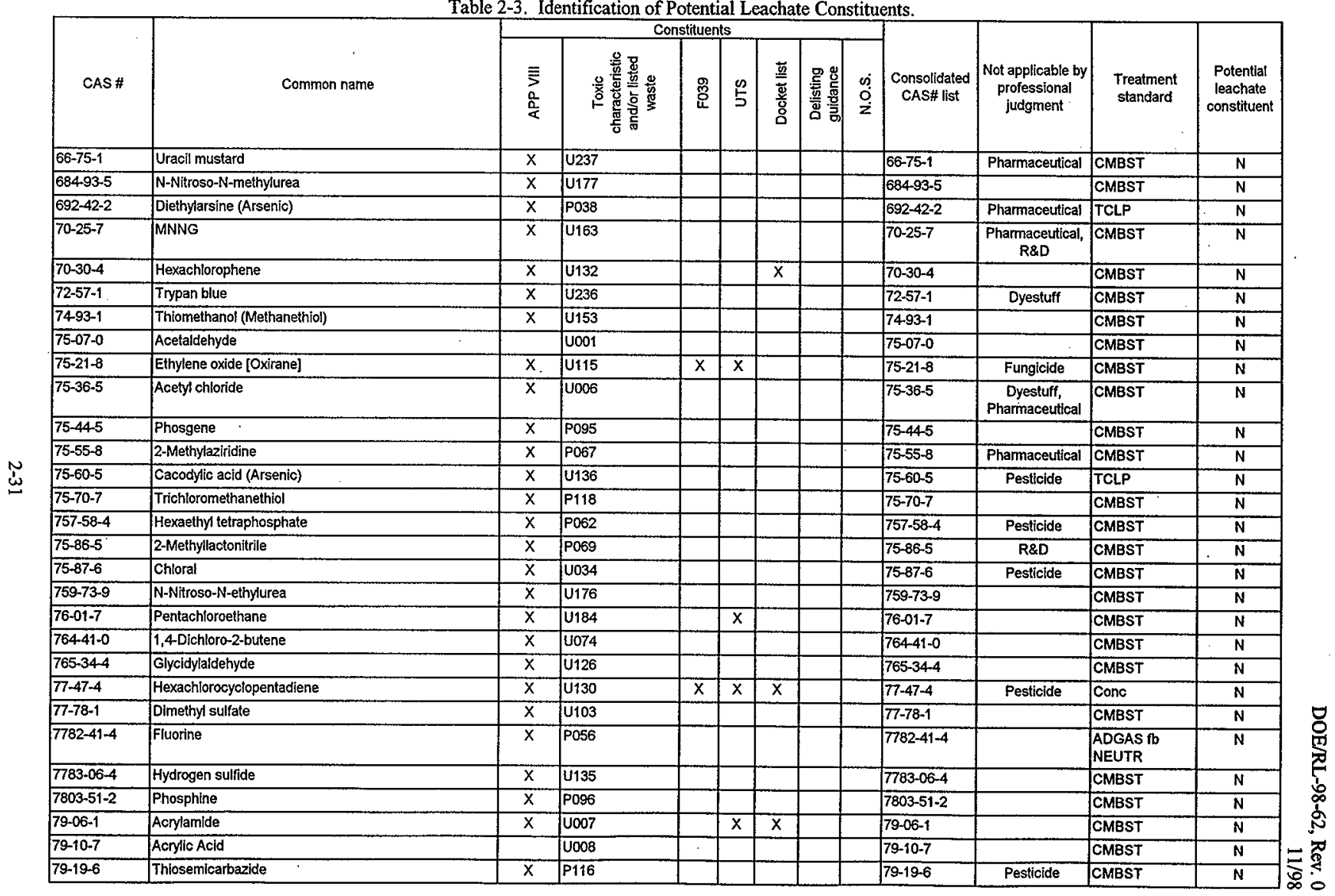


Table 2-3. Identification of Potential Leachate Constituents.

\begin{tabular}{|c|c|c|c|c|c|c|c|c|c|c|c|c|}
\hline & & & $\mathrm{Co}$ & tituen & & & & & & & & \\
\hline $\begin{array}{c}\text { CAS \# } \\
\\
\ldots \ldots\end{array}$ & 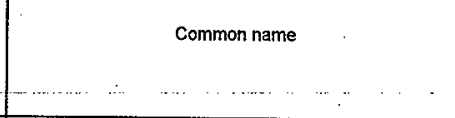 & $\underset{\substack{\frac{a}{a} \\
\frac{a}{\alpha}}}{\equiv}$ & 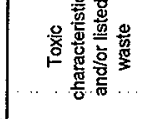 & 용 & $\stackrel{\infty}{5}$ & 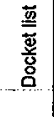 & 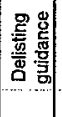 & $\begin{array}{l}\dot{j} \\
\dot{0} \\
z \\
z\end{array}$ & $\begin{array}{c}\text { Consolidated } \\
\text { CAS\# list } \\
\ldots\end{array}$ & $\begin{array}{c}\text { Not applicable by } \\
\text { professional } \\
\text { judgment }\end{array}$ & $\begin{array}{l}\text { Treatment } \\
\text { standard } \\
\end{array}$ & $\begin{array}{c}\text { Potential } \\
\text { leachate } \\
\text { constituent }\end{array}$ \\
\hline $79-22-1$ & Methyl chlorocarbonate & $x$ & U156 & & & & & & $79-22-1$ & Pesticide, Military & CMBST & $\vec{N}$ \\
\hline $79-44-7$ & Dimethylcarbamoyl chloride & $x$ & U097 & & & & & & $79-44-7$ & & CMBST & $\mathrm{N}$ \\
\hline $79-46-9$ & 2-Nittropropane & $\mathrm{X}$ & $F 005$, U17t & & & $\mathrm{X}$ & & & $79-46-9$ & & CMBST & $N$ \\
\hline $80-15-9$ & aipha, alpha-Dimethyl benzyl hydroperoxide & & U096 & & & & & & $80-15-9$ & & CMBST & $\mathbf{N}$ \\
\hline $81-07-2$ & Saccharin and Saccharin salts & $x$ & $\mathrm{U} 202$ & & & & & & $81-07-2$ & Consumer & CMBST & $\dot{\mathbf{N}}$ \\
\hline $81-81-2$ & Warfarin salts & $x$ & P001, U248 & & & & & & $81-81-2$ & & CMBST & $\mathbf{N}$ \\
\hline $823-40-5$ & Toluene-2,6-diamine & $\bar{x}$ & & & & $\bar{x}$ & & & $823-40-5$ & Dyestuff, Polymer & not specified & $\mathrm{N}$ \\
\hline $86-74-8$ & Carbazole & & $?$ & & & $x$ & & & $86-74-8$ & Dyestuff & not specified & $\mathbf{N}$ \\
\hline $86-88-4$ & alpha-Naphthylthiourea & $x$ & P072 & & & & & & $86-88-4$ & & CMBST & $\mathbf{N}$ \\
\hline $91-59-8$ & beta-Naphthylamine & $x$ & U168 & $\mathrm{x}$ & $x$ & $x$ & & & $91-59-8$ & & CMBST & $N$ \\
\hline $91.94-1$ & 3,3'-Dichlorobenzidine & $\bar{x}$ & U073 & & & $\mathrm{x}$ & & & $91-94-1$ & & CMBST & $N$ \\
\hline $92-87-5$ & Benzidine & $x$ & U021 & & & $\bar{x}$ & & & $92-87-5$ & & CMBST & $N$ \\
\hline $94-11-1$ & 2,4-D salts \& esters & $x$ & D016, U240 & & & & & & $94-11-1$ & & CMBST & $N$ \\
\hline $94-58-6$ & Dihydrosafrole & $x$ & U090 & & & & & & $94-58-6$ & Consumer & CMBST & $N$ \\
\hline $95-06-7$ & Sulfallate & $\underline{x}$ & & & & & & & $95-06-7$ & Pesticide & not specified & $\mathrm{N}$ \\
\hline $95-53-4$ & o-Toluidine & $x$ & U328 & & & $x$ & & & $95-53-4$ & & CMBST & $N$ \\
\hline $96-45-7$ & Ethylenethiourea & $\mathrm{x}$ & 4116 & & & & & & $96-45-7$ & $\cdot$ & CMBST & $N$ \\
\hline $97-74-5$ & Tetramethytthituram monosulfide & $x$ & & & & & & & $97-74-5$ & Polymer & not specified. & $\mathrm{N}$ \\
\hline $98-01-1$ & Furfural & & $\mathrm{U} 125$ & & & & & & $98-01-1$ & $\dot{.}$ & CMBST & $\mathbf{N}$ \\
\hline $98-07-7$ & Benzotrichloride & $\bar{x}$ & บ023 & & & & & & $98-07-7$ & & CMBST & $\mathbf{N}$ \\
\hline $98-09-9$ & Benzenesulfonyl chloride & & U020 & & & & & & $98-09-9$ & & CMBST & $\mathbf{N}$ \\
\hline $98-82-8$ & Cumene & & U055 & & & $x$ & & & $98-82-8$ & & CMBST & $\mathbf{N}$ \\
\hline $98-87-3$ & Benzal chloride & $x$ & U017 & & $\bar{x}$ & & & & $98-87-3$ & & CMBST & $\mathrm{N}$ \\
\hline $99-35-4$ & 1,3,5-Trinitrobenzene & $x$ & 10234 & & & $x$ & & & $99-35-4$ & Military & CMBST & $\mathrm{N}$ \\
\hline
\end{tabular}




\subsection{AREA EFFLUENT TREATMENT FACILITY AND LIQUID EFFLUENT RETENTION FACILITY PROCESS}

3

4

The primary purpose of this delisting petition modification is to enable the discharge of treated effluent from the ETF to a SALDS. This chapter describes the LERF and ETF processes. The controls that are built into the ETF to ensure that the effluent meets the Washington State Waste Discharge Permit ST 4500 and the limits contained in the Final Delisting also are described.

\subsection{LIQUID EFFLUENT RETENTION FACILITY PROCESS DESCRIPTION}

LERF is a RCRA final status waste management unit. This waste management unit is a RCRA compliant surface impoundment operating pursuant to the Treatment Surface Impoundment Exemption provisions of 40 CFR 268.4. Each of the three LERF basins has an operating capacity of 29.5-million liters. The LERF receives aqueous waste through several inlets including the following:

- A pipeline that connects LERF with the 242-A Evaporator (another RCRA final status waste management unit)

- A pipeline from the 200 West Area

- A pipeline that connects LERF to the Load-In Station at ETF

- A series of sample ports located at each basin.

Figure 3-1 presents a general layout of LERF and associated pipelines.

Aqueous waste from LERF is pumped to the ETF through one of two double-walled fiberglass transfer pipelines. Effluent from the ETF also can be transferred back to the LERF through one of these transfer pipelines. These pipelines are equipped with leak detection located in the annulus between the inner and outer pipes. In the event that these leak detectors are not in service, the pipelines visually are inspected during transfers for leakage by opening the secondary containment drain lines at the ETF end of the transfer pipelines.

Each basin is equipped with six available sample risers constructed of 6-inch perforated pipe. A seventh sample riser in each basin is dedicated to influent aqueous waste receipt piping (except for aqueous waste received from the 242-A Evaporator), and an eighth riser in each basin contains liquid level instrumentation. Each riser extends along the sides of each basin from the top to the bottom of the basin and allow samples to be collected from any depth. Personnel access to these sample ports is from the perimeter area of the basins.

A catch basin is provided at the northwest comer of each LERF basin for aboveground piping and manifolds for transfer pumps. Aqueous waste from the 242-A Evaporator is transferred through piping that ties into piping at the catch basins. Under routine operations, a submersible pump is used to transfer aqueous waste from a LERF basin to the ETF for processing or for basin-to-basin transfers. This pump is connected to a fixed manifold on one of four available risers.

Each basin consists of a multilayer liner system supported by a concrete anchor wall around the basin perimeter and a soil-bentonite clay underlayment. The multilayer liner system consists of a primary liner in contact with the aqueous waste, a layer of bentonite carpet, a geonet, a geotextile, a gravel layer, and a secondary liner that rests on the bentonite underlayment. Any aqueous waste leakage through the primary liner flows through the geonet to a leachate collection system. The leachate flows to a sump at the northwest comer of each basin, where the leachate is pumped up the sideslope and back into the basin 
above the primary liner. Each liner is constructed of high-density polyethylene. A floating cover made of very low-density polyethylene is stretched over each basin above the primary liner. These covers serve to keep unwanted material from entering the basins, and to minimize evaporation of the liquid contents.

\subsection{EFFLUENT TREATMENT FACILITY PROCESS DESCRIPTION}

ETF is a RCRA final status waste management unit. This waste management unit is a RCRA compliant tank system with container storage capabilities. The ETF is designed as a flexible treatment system that provides treatment for contaminants anticipated in process condensate and other aqueous waste. The design influent flow rate into the ETF is approximately 570 liters per minute with planned outages for activities such as maintenance on the ETF systems. Maintenance outages typically are scheduled between treating a batch of aqueous waste, referred to as treatment campaigns. The effluent flow (or volume) is equivalent to the influent flow (or volume).

The ETF generally receives aqueous waste directly from the LERF. However, aqueous waste also can be transferred from the Load-In Station to the ETF? Aqueous waste is treated and stored in the ETF process area in a series of process units. Within the ETF, waste also is stored in containers. Figure 3-2 provides the relative locations of the process area and container storage areas within the ETF.

The process units are grouped in either the primary or the secondary treatment train. The primary treatment train provides for the removal or destruction of contaminants. Typically; the secondary treatment train processes the waste by-products from the primary treatment train by reducing the volume of waste. In the secondary treatment train, contaminants are concentrated and dried to a powder. The liquid fraction is routed back to the primary treatment train. Figure 3-3 presents the ETF floor plan, the relative locations of the individual process units and associated tanks within the ETF, and the location of the Load-In Station.

The dry powder waste and maintenance and operations waste are containerized and stored in the container storage area or in collection areas. Secondary containment is provided for all ETF container and tank systems (including ancillary equipment).

In the following sections, several figures are provided that present general illustrations of the treatment units and the relation to the process.

The ETF receives aqueous waste from LERF or the Load-In Station. The ETF Load-In Station, located due east of the surge tank and outside of the perimeter fence (Figure 3-3), was designed and constructed to provide the capability to unload, store, and transfer aqueous waste to the ETF or LERF from tanker trucks, and potentially other containers (such as drums). Currently, tanker trucks are used to unload aqueous waste at the Load-In Station.

\subsubsection{Effluent Treatment Facility Operating Configuration}

41 Because the operating configuration of the ETF can be adjusted or modified, aqueous waste streams can be effectively treated to below Final Delisting and Discharge Permit limits. The operating configuration of the ETF depends on the unique chemistry of an aqueous waste stream(s). Before an aqueous waste stream is accepted for treatment, the waste is characterized and evaluated. Information from the characterization is used to adjust the treatment process or change the configuration of the ETF process units, as necessary, to optimize the treatment process for a particular aqueous waste stream.

Typically, an aqueous waste is processed first in the primary treatment train, where the ETF is configured to process an aqueous waste through the ultraviolet oxidation (UV/OX) unit first, followed by the reverse 
osmosis (RO) unit. However, under an alternate configuration, an aqueous waste could be processed in the RO unit first. For example, high concentrations of nitrates in an aqueous waste might interfere with the performance of the UV/OX. In this case, the ETF could be configured to process the waste in the RO unit before the UV/OX unit.

The flexibility of the ETF also allows for some aqueous waste to be processed in the secondary treatment train first. For example, for small volume aqueous waste with high concentrations of some anions and metals, the approach could be to first process the waste stream in the secondary treatment train. This approach would prevent premature fouling or scaling of the RO unit. The liquid portion (i.e., untreated overheads from the ETF evaporator and thin film dryer) would be sent to the primary treatment train.

Figures 3-4 and 3-5 provide example process flow diagrams for two different operating configurations.

\subsubsection{Primary Treatment Train}

The primary treatment train consists of the following units:

- Surge tank - inlet, surge capacity

- Filtration - for suspended solids removal

- UV/OX - organic destruction

- $\mathrm{pH}$ adjustment - waste neutralization

- Hydrogen peroxide decomposition - removal of excess hydrogen peroxide

- Degasification - removal of carbon dioxide

- RO - removal of dissolved solids and radionuclides

- Ion exchange (IX) - removal of dissolved solids and radionuclides

- Verification - holding tanks during verification.

Each of the primary treatment train process units and ancillary systems provides treatment for removal or destruction of various constituents. The primary treatment train units are operated as needed in different configurations, às determined by the characteristics of an aqueous waste stream, to protect ETF equipment and to meet discharge requirements.

Influent Receipt/Surge Tank. Depending on the configuration of the ETF, the surge tank is one inlet used to feed an aqueous waste into the ETF for treatment. In Configuration 1 (Figure 3-4), the surge tank is the first component downstream of the LERF. The surge tank provides a storage/surge volume for chemical pretreatment and controls feed flow rates from the Load-In Station or LERF to the ETF. However, in Configuration 2 (Figure 3-5), aqueous waste from LERF is fed directly into the treatment units. In this configuration, the surge tank receives aqueous waste that has been processed in the RO units and provides the feed stream to the remaining downstream process units. In yet another configuration, some small volume aqueous waste could be received into the secondary treatment train first for processing. In this case, the aqueous waste would be received directly into the secondary waste receiving tanks. Finally, the surge tank also receives waste extracted from various systems within the primary and secondary treatment train while in operation.

The surge tank is located outside the ETF on the south side. In the surge tank (Figure 3-6), the pH of an aqueous waste is adjusted using the metered addition of sulfuric acid and sodium hydroxide, as necessary, to prepare the waste for treatment in downstream processes. In addition, hydrogen peroxide or biocides could be added to control biological growth in the surge tank. A pump recirculates the contents in the surge tank, mixing the chemical reagents with the waste to a uniform $\mathrm{pH}$. 
Filtration. Two primary filter systems remove suspended particles in an aqueous waste: a rough filter removes the larger particulates, while a fine filter removes the smaller particulates. The location of these filters depends on the configuration of the primary treatment train. However, the filters normally are located upstream of the RO units.

The solids accumulating on these filter elements are backwashed to the secondary waste receiving tanks with pulses of compressed air and water, forcing water back through the filter. The filters are cleaned. chemically when the backwashing process does not facilitate acceptable filter performance.

Auxiliary fine and rough filters (e.g., disposable filters) have been installed to provide additional filtration capabilities Depending on the configuration of the ETF, the auxiliary filters are operated either in series with the primary filters to provide additional filtration or in parallel, instead of the primary fine and rough filters, to allow cleaning of the primary fine and rough filters while the primary treatment train is in operation.

Ultraviolet Light/Oxidation. Organic compounds contained in an aqueous waste stream are destroyed in the UV/OX system (Figure 3-7). Hydrogen peroxide is mixed with the waste. The UV/OX system uses the photochemical reaction of UV light on hydrogen peroxide to form hydroxyl radicals and other reactive species that oxidize the organic compounds. The final products of the complete reaction are carbon dioxide, water, and inorganic ions.

Organic destruction is accomplished in two UV/OX units operating in parallel. During the UV/OX process, the aqueous waste passes through reaction chambers where hydrogen peroxide is added. While in the UV/OX system, the temperature of an aqueous waste is monitored. Should the temperature of the waste exceed the upper limits for the UV/OX or RO systems, heat exchangers are used to reduce the temperature of the waste.

pH Adjustment. The $\mathrm{pH}$ of a waste stream is monitored and controlled at different points throughout the treatment process. Within the primary treatment train, the $\mathrm{pH}$ of a waste can be adjusted with sulfuric acid or sodium hydroxide to optimize operation of downstream treatment processes or adjusted before final discharge. For example, the $\mathrm{pH}$ of an aqueous waste would be adjusted in the $\mathrm{pH}$ adjustment tank after the UV/OX process and before the $\mathrm{RO}$ process. In this example, $\mathrm{pH}$ is adjusted to cause certain chemical species such as ammonia to form ammonium sulfate, thereby increasing the rejection rate of the RO.

Hydrogen Peroxide Decomposition. Typically, hydrogen peroxide added into the UV/OX system is not consumed completely by the system. Because hydrogen peroxide is an oxidizer that can damage ETF equipment, the residual hydrogen peroxide from the UV/OX system is removed to protect downstream equipment. The hydrogen peroxide decomposer uses activated carbon to break down the hydrogen peroxide that is not consumed completely in the process of organic destruction. The aqueous waste is sent through a column of fluidized activated carbon that breaks down the hydrogen peroxide into water and oxygen. The gas generated by the decomposition of the hydrogen peroxide is vented to the vessel offgas system.

Degasification. The degasification column is used to purge dissolved carbon dioxide from the aqueous waste to reduce the carbonate loading to downstream dissolved solids removal processes within the ETF primary treatment train. The purged carbon dioxide is vented to the vessel offgas system.

Reverse Osmosis. The RO system (Figure 3-8) uses pressure to force clean water molecules through semi-permeable membranes while keeping the larger molecule contaminants, such as dissolved solids, radionuclides, and large molecular weight organic materials, in the membrane. The RO process uses a staged configuration to maximize water recovery. The process produces two separate streams, including a 
clean 'permeate' and a concentrate (or retentate), which are concentrated as much as possible to minimize the amount of secondary waste produced.

The RO process is divided into first and second stages. Aqueous waste is fed to the first RO stage from the RO feed tank. The secondary waste receiving tanks of the secondary treatment train receive the retentate removed from the first RO stage, while the second RO stage receives the permeate (i.e., 'treated' aqueous waste from the first RO stage). In the second RO stage, the retentate is sent to the first stage RO feed tank while the permeate is sent to the IX system or to the surge tank, depending on the configuration of the ETF.

Two support systems facilitate this process. An anti-scale system injects scale inhibitors as needed into the feed waste to prevent scale from forming on the membrane surface. A clean-in-place system using cleaning agents, such as descalants and surfactants, cleans the membrane pores of surface and subsurface deposits that have fouled the membranes.

Ion Exchange. Because the RO process removes most of the dissolved solids in an aqueous waste, the IX process (Figure 3-9) act as a polishing unit. The IX system consist of three columns containing beds of cation and/or anion resins. This system is designed to allow for regeneration of resins and maintenance of one column while the other two are in operation. Though the two columns generally are operated in series, the two columns also can be operated in parallel or individually.

Typically, the two columns in operation are arranged in a primary/secondary (lead/lag) configuration, and the third (regenerated) column is maintained in standby. When dissolved solids breakthrough the first IX column and are detected by a conductivity sensor, this column is removed from service for regeneration, and the second column replaces the first column and the third column is placed into service. The column normally is regenerated using sulfuric acid and sodium hydroxide. The resulting regeneration waste is collected in the secondary waste receiving tanks.

Should regeneration of the IX resins become inefficient, spent resins are transferred into a disposal container. The container is designed to provide dewatering with remote monitoring of the resin and water levels within the container. Displaced air from the vessels is exhausted through an entrainment separator (to remove water drops) and a high-efficiency particulate air filter and into the vessel offgas system. Water is removed from the container to the degree possible and retumed to the surge tank. Dewatered resins are transferred to a final storage/disposal point.

Verification. The three verification tanks (Figure 5-10) are used to hold the treated effluent while a determination is made that the effluent meets discharge limits. Should a treated effluent not meet Washington State Waste Discharge Permit or Final Delisting requirements, the effluent can be returned to the primary treatment train for additional treatment or to the LERF.

The three verification tanks alternate between three operating modes: receiving treated effluent, holding treated effluent during laboratory analysis and verification, or discharging verified effluent. Treated effluent also can be returned to the ETF to provide 'clean' service water for operational and maintenance functions, e.g., for boiler water and for backwashing the filters. This recycling keeps the quantity of fresh water used to a minimum.

\subsubsection{Secondary Treatment Train}

The secondary treatment system typically receives and processes the following by-products generated from the primary treatment train: concentrate from the first RO stage, filter backwash, regeneration waste from the LX system, and spillage or overflow received into the process sumps. Depending on the operating 
1 configuration, however, some aqueous waste could be processed in the secondary treatment train before the

2 primary treatment train (refer to Figures 3-4 and 3-5 for example operating configurations).

The secondary treatment train provides the following processes:

- Secondary waste receiving - tank receiving

- Evaporation - concentrates secondary waste streams

- Concentrate staging - concentrate receipt and $\mathrm{pH}$ adjustment in concentrate tanks

- Thin film drying - dewatering of secondary waste streams

- Container handling - packaging of dewatered secondary waste.

Secondary Waste Receiving. Waste to be processed in the secondary treatment train is received into two secondary waste receiving tanks, where the $\mathrm{pH}$ can be adjusted with sulfuric acid or sodium hydroxide for optimum evaporator performance.

Evaporation. The ETF evaporator is the first step in the secondary treatment train to reduce the volume of secondary waste. The ETF evaporator is fed alternately by the two secondary waste receiving tanks. One tank serves as a waste receiver while the other tank is operated as the feed tank. The ETF evaporator vessel (also referred to as the vapor body) is the principal component of the evaporation process (Figure 3-11).

Feed from the secondary waste receiving tanks is pumped through a heater to the recirculation loop of the ETF evaporator. In this loop, concentrated waste is recirculated from the ETF evaporator, to a heater, and back into the evaporator where vaporization occurs. As water leaves the evaporator system in the vapor phase, the concentration of the waste in the evaporator increases. When the concentration of the waste reaches the appropriate density, a portion of the concentrate is pumped to one of the concentrate tanks.

The vapor that is released from the ETF evaporator is routed to the entrainment separator, where water droplets and/or particulates are separated from the vapor. The 'cleaned' vapor is routed to the vapor compressor and heater. The steam from the vapor compressor/heater is used to heat the recirculating concentrate in the ETF evaporator. From the vapor compressor/heater, the steam is condensed and fed to the distillate flash tank, where the saturated condensate received from the heater drops to atmospheric pressure and cools to the normal boiling point through partial flashing (rapid vaporization caused by a pressure reduction). The resulting distillate is routed to the surge tank. Noncondensible vapors, such as air, are exhausted by a vacuum blower to the vessel offgas system.

Concentrate Staging. The concentrate tanks make up the head end of the thin film drying process. From the ETF evaporator, concentrate is pumped into two concentrate tanks and $\mathrm{pH}$ adjusted. The concentrate tanks function alternately between concentrate receiver and feed tank for the thin film dryer.

Thin Film Drying. The thin film dryer provides the second step in reducing the volume of secondary waste generated by the ETF treatment process. From the concentrate tanks, feed is pumped through a preheater to the thin film dryer (Figure 3-12) that is heated by steam. As the concentrated waste flows down the length of the dryer, the waste is dried. The dried film, or powder, is scraped off the dryer cylinder by blades attached to a rotating shaft. The powder is funneled through a cone-shaped powder hopper at the bottom of the dryer and into the Container Handling System.

Overhead vapor released by the drying of the concentrate is condensed in the distillate condenser. Excess heat is removed from the distillate by a water-cooled heat exchanger. Part of the distillate is circulated 
1 . back to the condenser spray nozzles. The remaining distillate is pumped to the surge tank. Any

2 noncondensible vapors and particulates from the spray condenser are exhausted to the vessel offgas system.

4 Container Handling. Before an empty container is moved into the Container Handling System

5 (Figure 3-13), the lids are loosely placed on the containers and the container is placed on a conveyor. After 6 the lid is removed, the containers are moved into the container filling area after passing through an air lock.

7 The empty container is located under the thin film dryer, and raised into position. The container is sealed

8 to the thin film dryer and a rotary valve begins the transfer of powder to the empty container. Air displaced

9 from the container is vented to the entrainment separator attached to the ETF evaporator that exhausts to

10 the vessel offgas system.

The container is filled to a predetermined level, recapped, and moved along the conveyor to the smear station airlock. At the smear station airlock, the container is moved onto the conveyor by remote control. The airlock is opened and the smear sample (surface wipe) is taken and the radionuclide contamination level counted. If the container has contaminated material on the outside, the container is moved to the washdown station and washed. The container wash water drains to sump tank 1. The washed container is air-dried and retested. Filled containers that pass the smear test are labeled, placed on pallets, and moved 


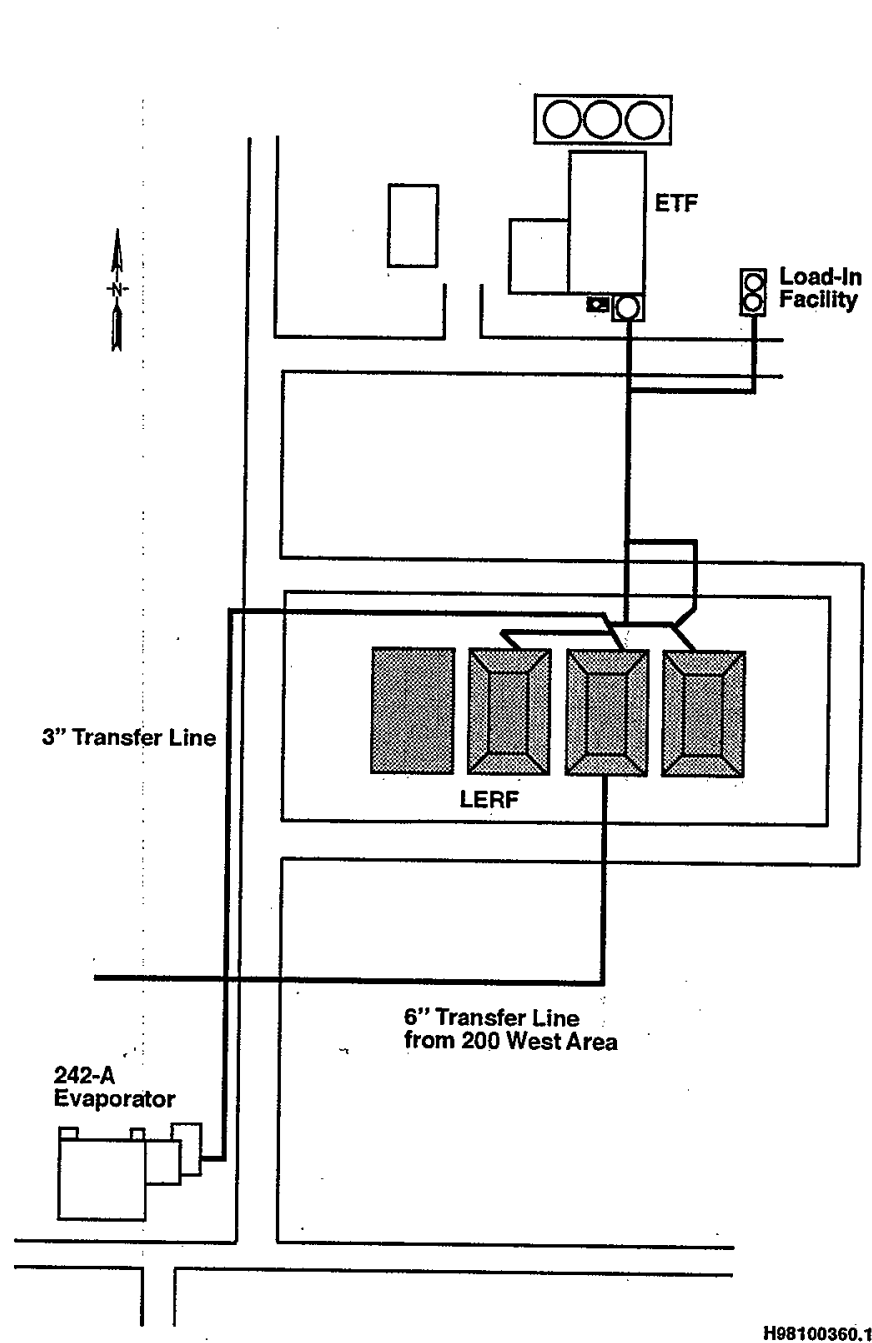

DOE/RL-98-62, Rev. 0

$11 / 98$

Figure 3-1. Liquid Effluent Retention Facility Layout. 


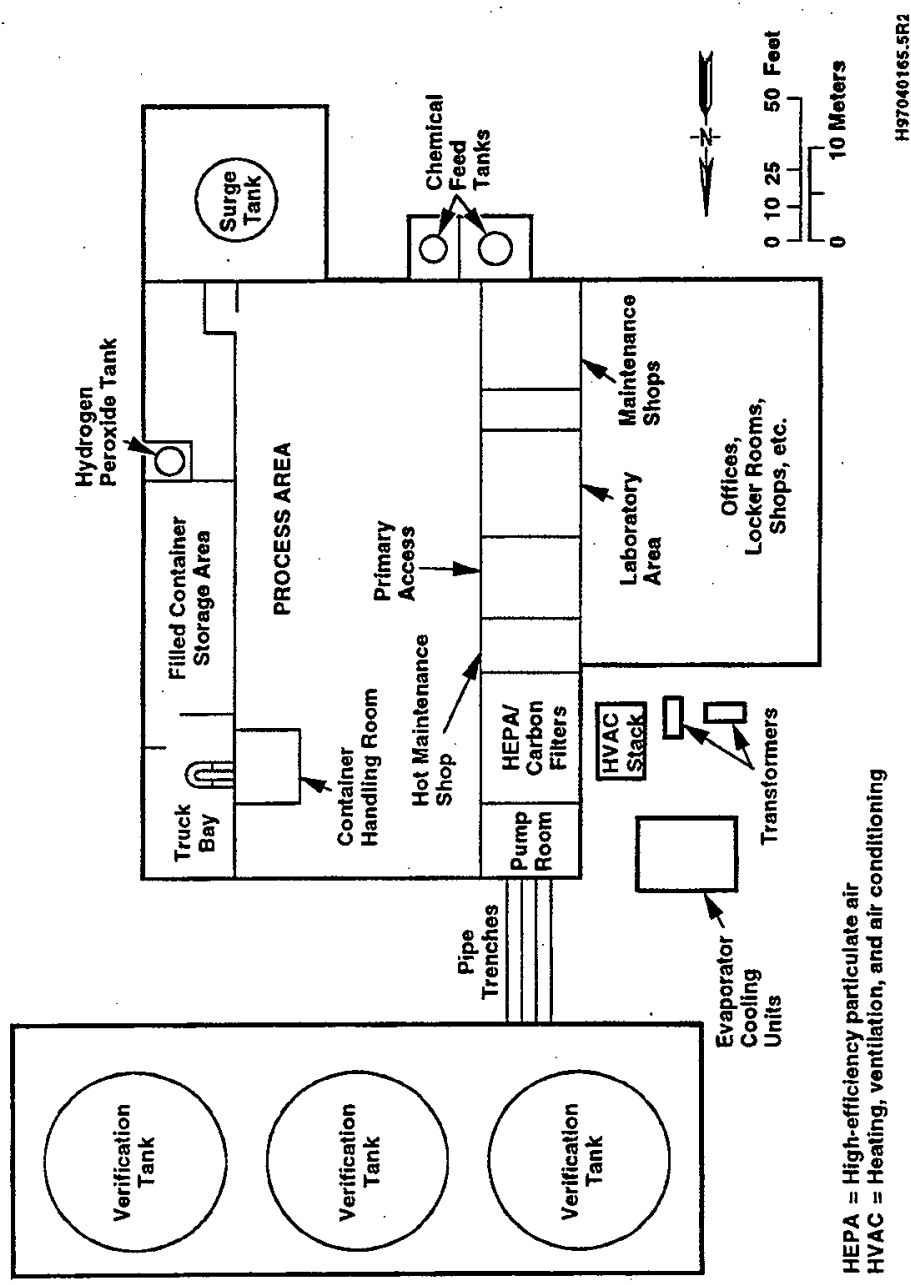

Figure 3-2. Plan View of the 200 Area Effluent Treatment Facility. 
DOE/RL-98-62, Rev. 0

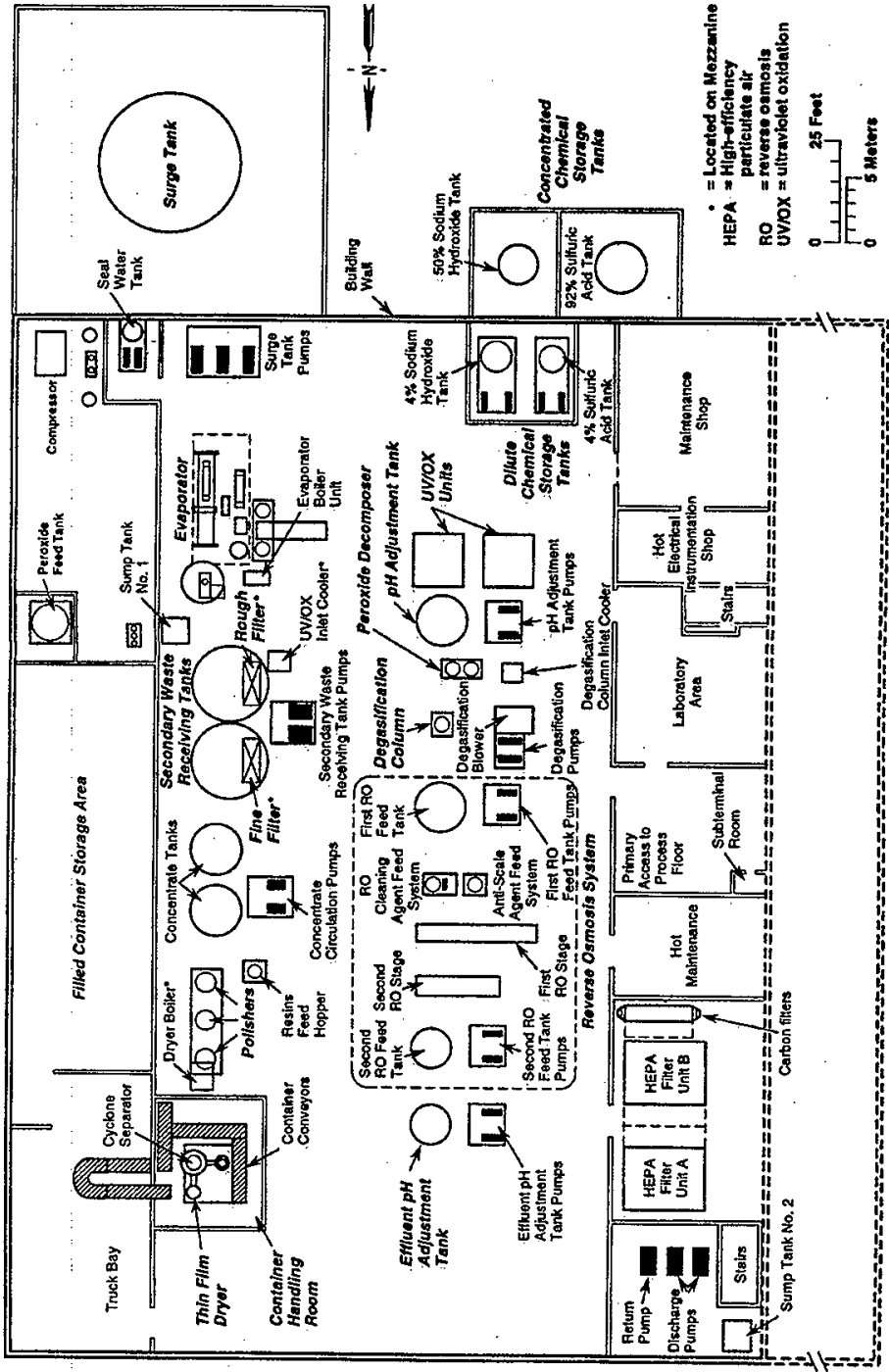

Figure 3-3. 200 Area Effluent Treatment Facility Layout. 
DOE/RL-98-62, Rev. 0

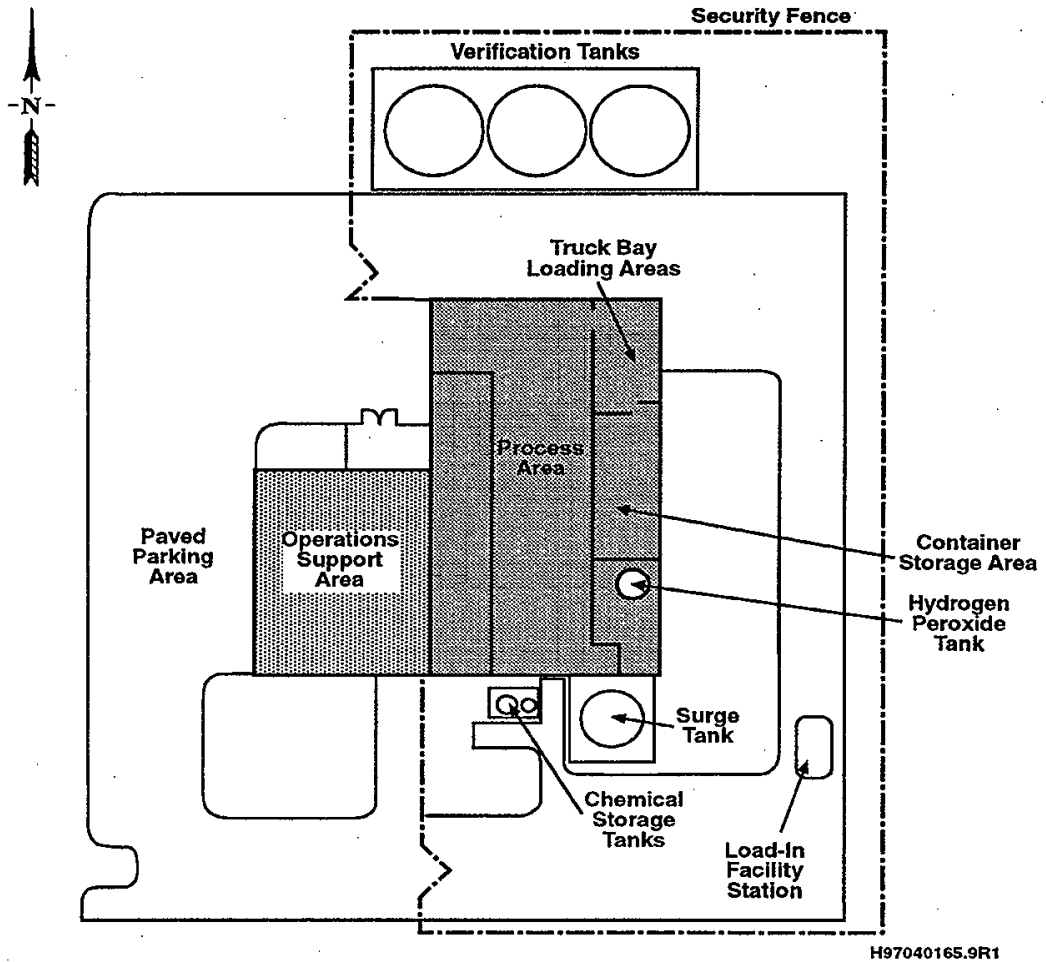

Figure 3-4. 200 Area Effluent Treatment Facility. 
DOE/RL-98-62, Rev. 0

$11 / 98$

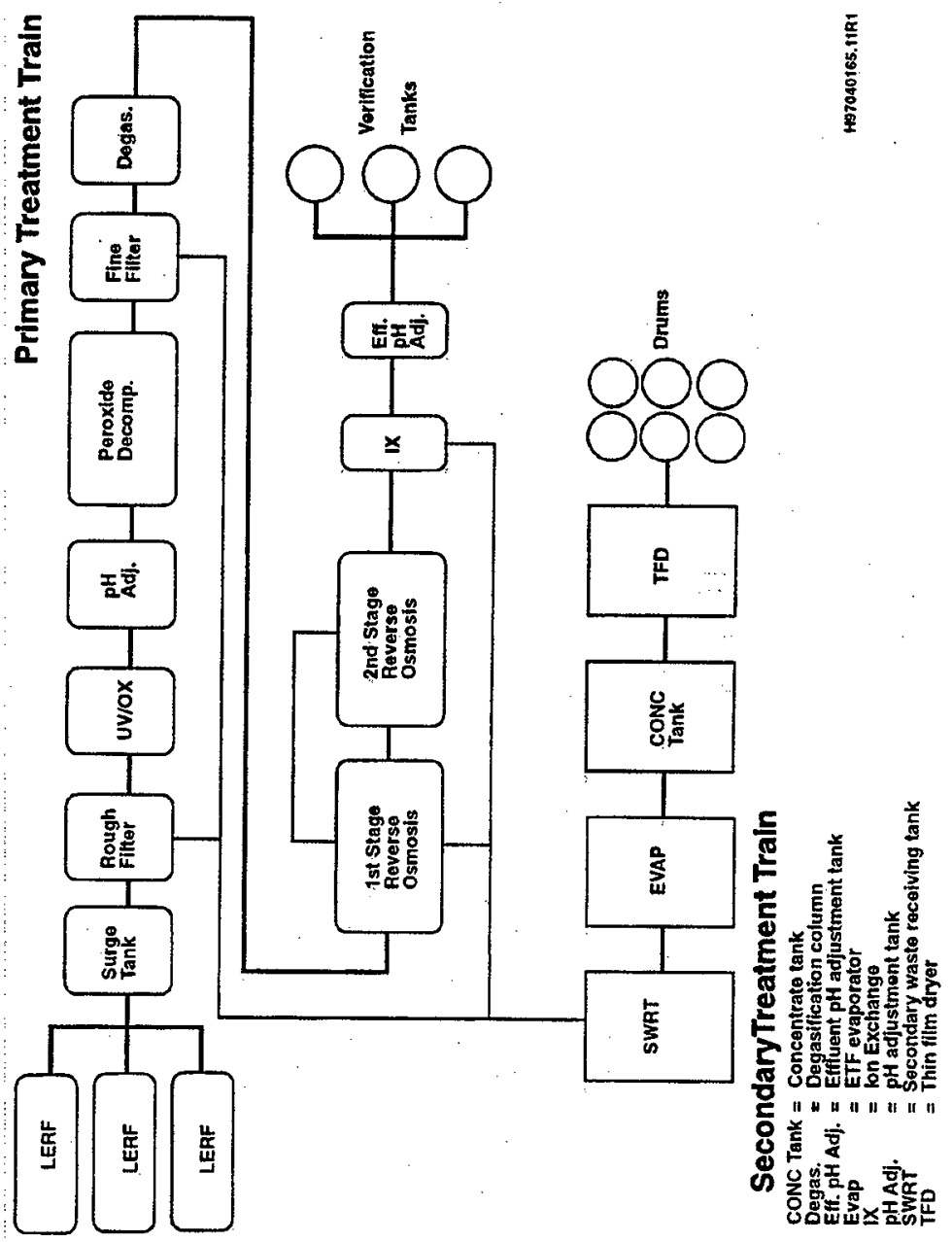

Figure 3-5. Example - 200 Area Effluent Treatment Facility Configuration 1. 


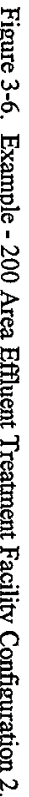

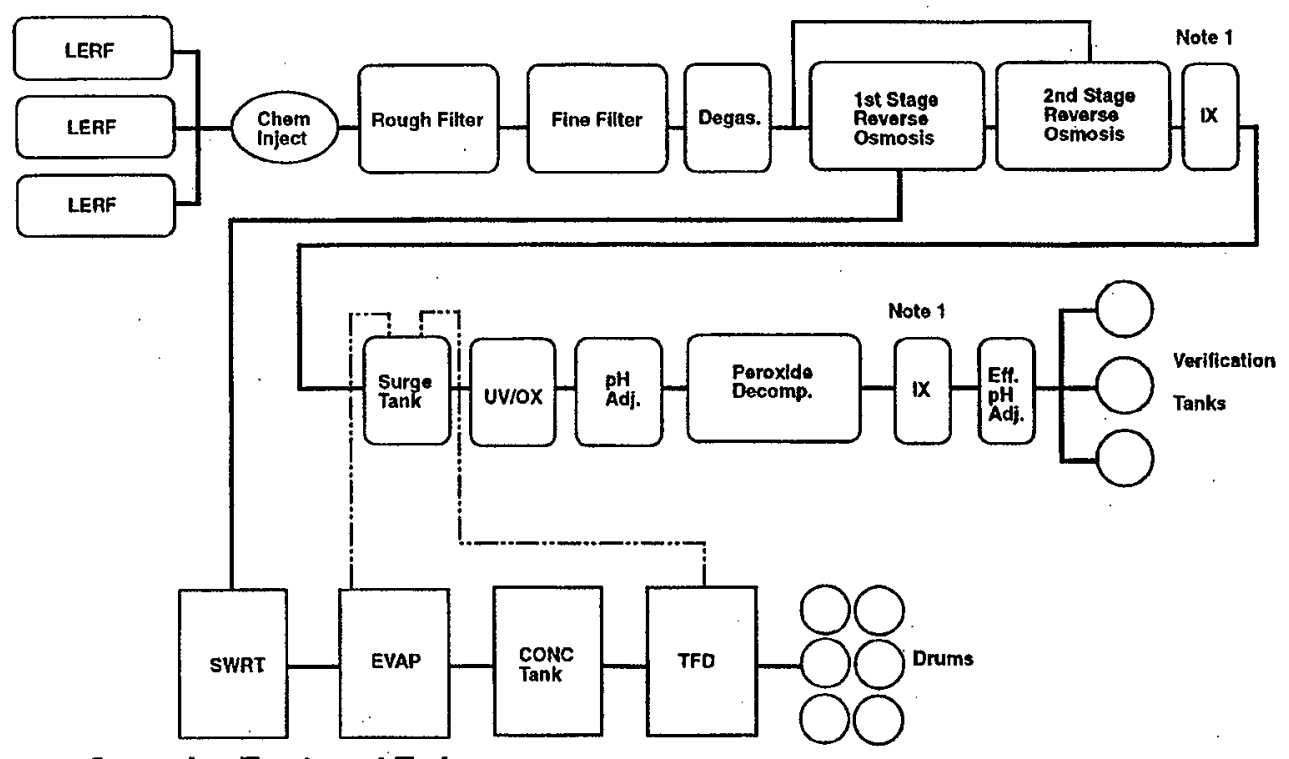

\section{SecondaryTreatment Train}

Note1: IX can be in either location

CONC Tank = Concentrate tank

Degas. $=$ Degasification column

Eff. pH Adf. = Effluent pH adjustment tank

Evap = ETF ovaporator

IX = lon exchange

$\mathrm{pH}$ Adj. $\quad=\mathrm{pH}$ adjustment tank

SWRT $\quad$ Secondary waste receiving tank

TFD = Thin film dryer 
Configuration \#2

2nd RO Permeate

Evaporator Distillate

Thin Film Dryer Distillate
Configuration \#1

LERF

Load In-Station

Polisher

Evaporator Distillate

Dryer Distillate

Sump \#2

Resin Dewatering

Chemical Reagent

Feed System
Surge Tank

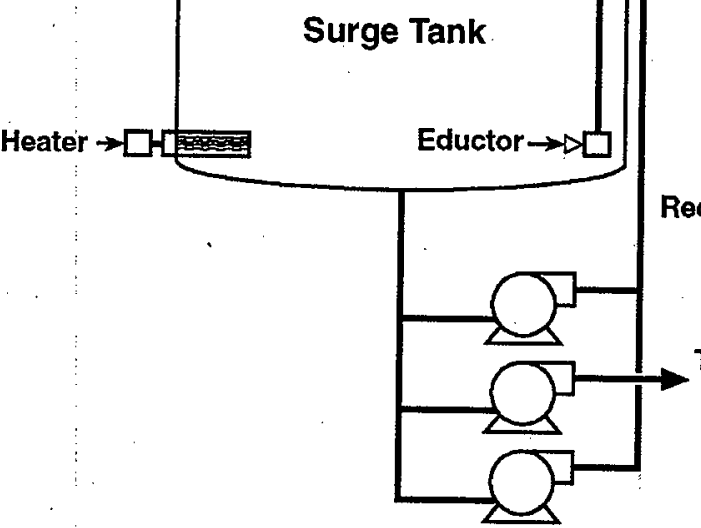

Surge Tank Pumps
Recirculation Line

TO: Rough

Filter (Conf \#1)

or

UV/OX (Conf \#2)

H97040165.19

R1

Figure 3-7. Surge Tank. 


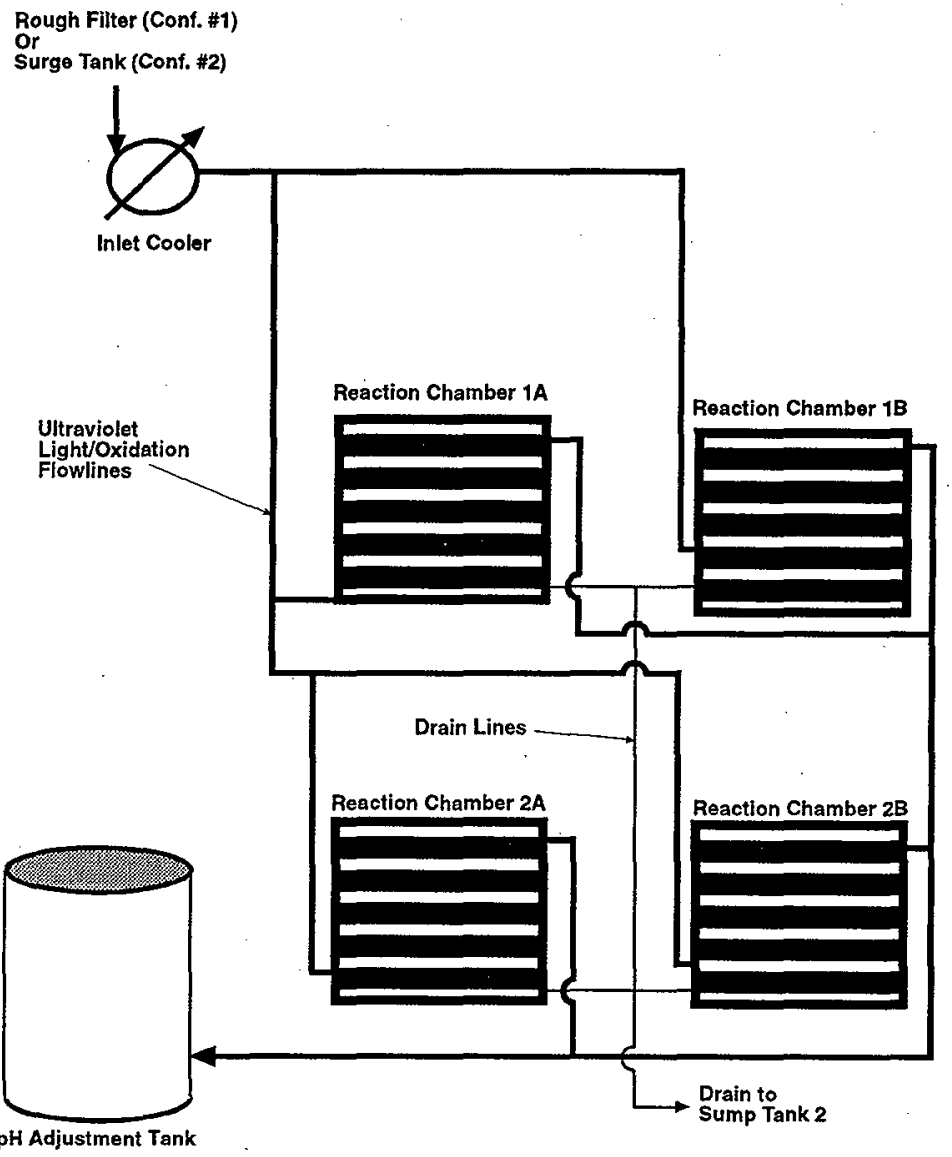

H97040165.20

Figure 3-8. Ultraviolet Light/Oxidation Unit. 


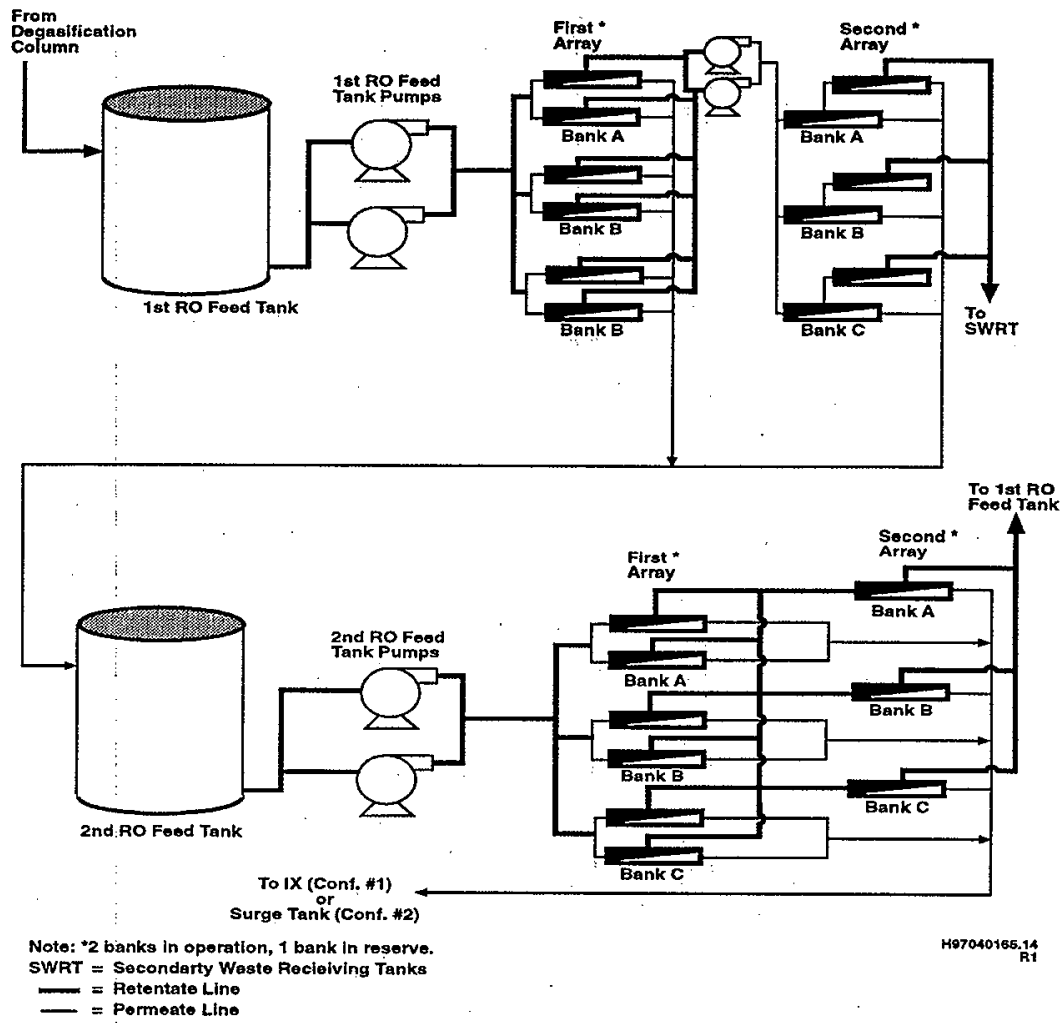

Figure 3-9. Reverse Osmosis Unit. 


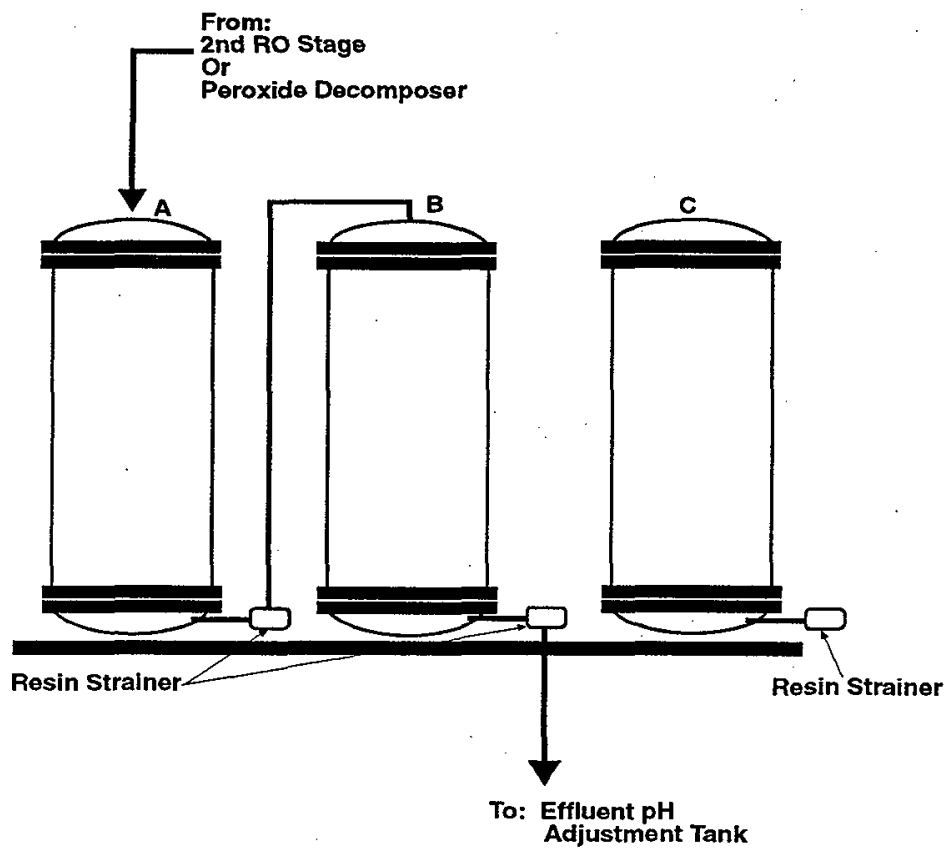

NOTE: Example Configuration-Column $A$ and $B$ in Operation, Column $\mathrm{C}$ in Standby Mode

H97040165.18

Figure 3-10. Ion Exchange Unit. 
From pH Adjustment Tank

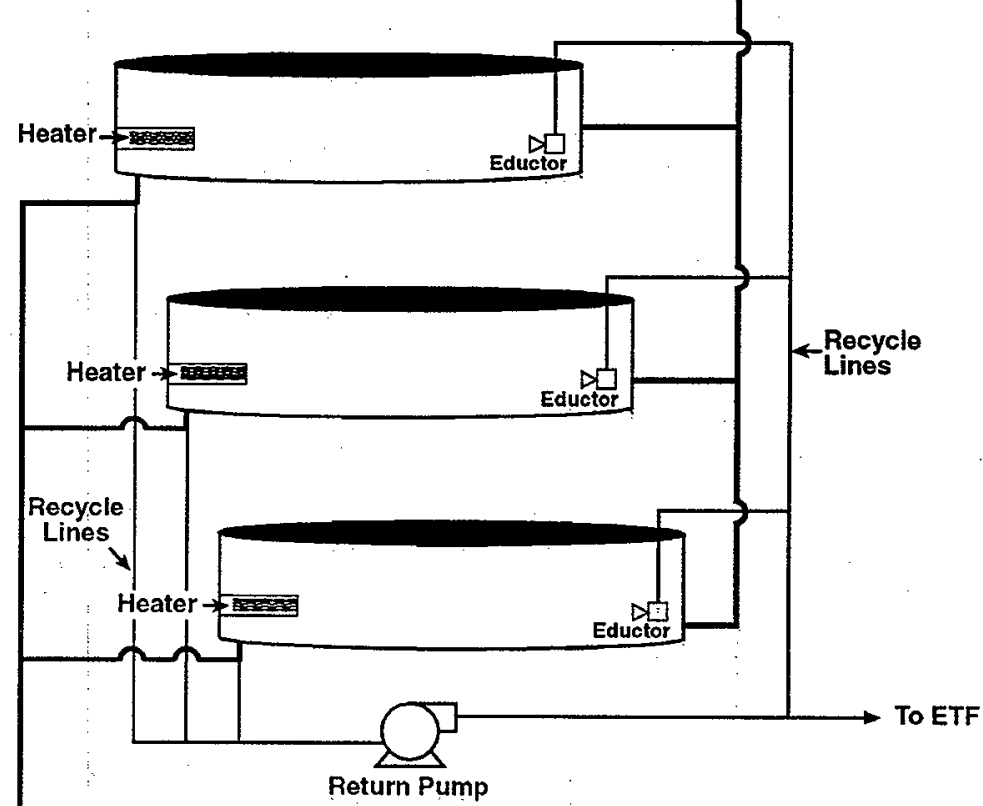

Transfer Pumps

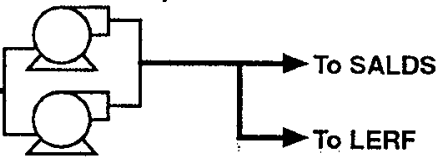

H97040165.17

Figure 3-11. Verification Tanks. 


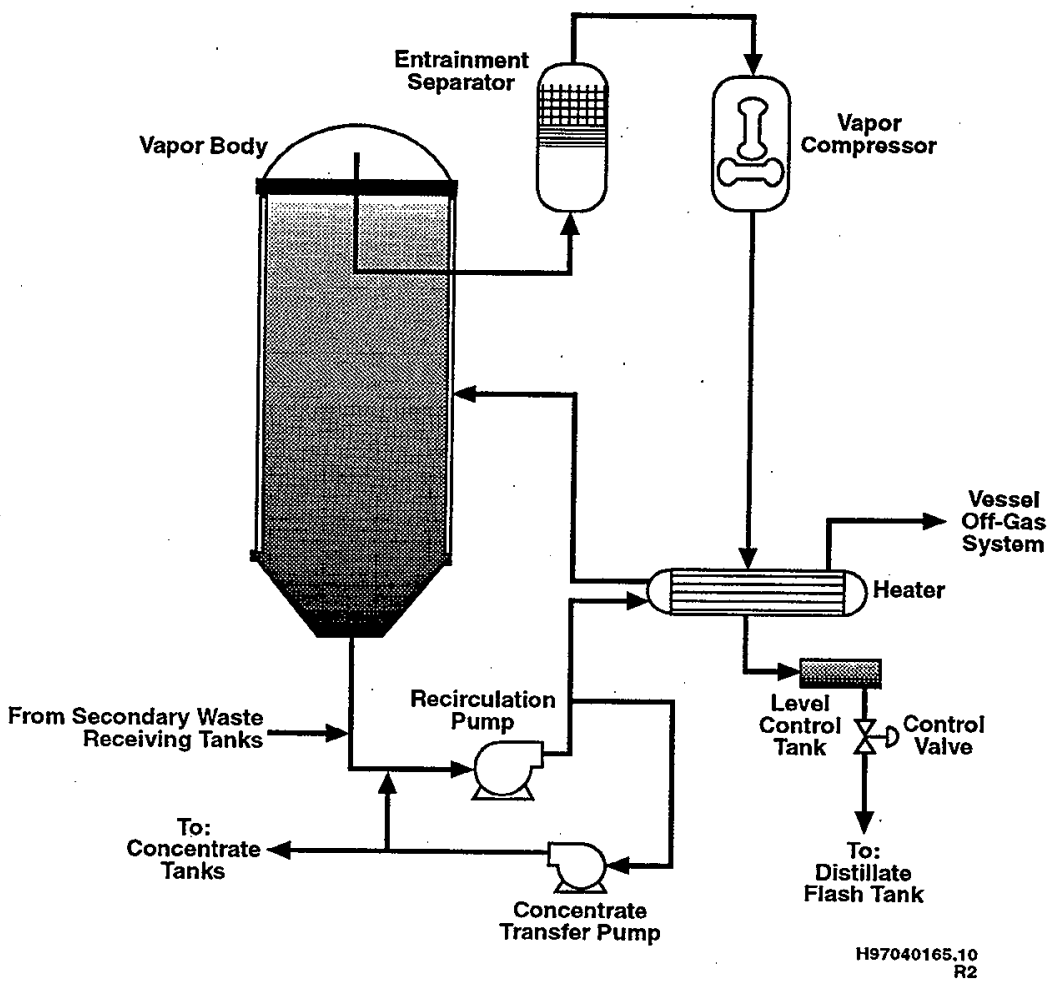

Figure 3-12. Effluent Treatment Facility Evaporator. 


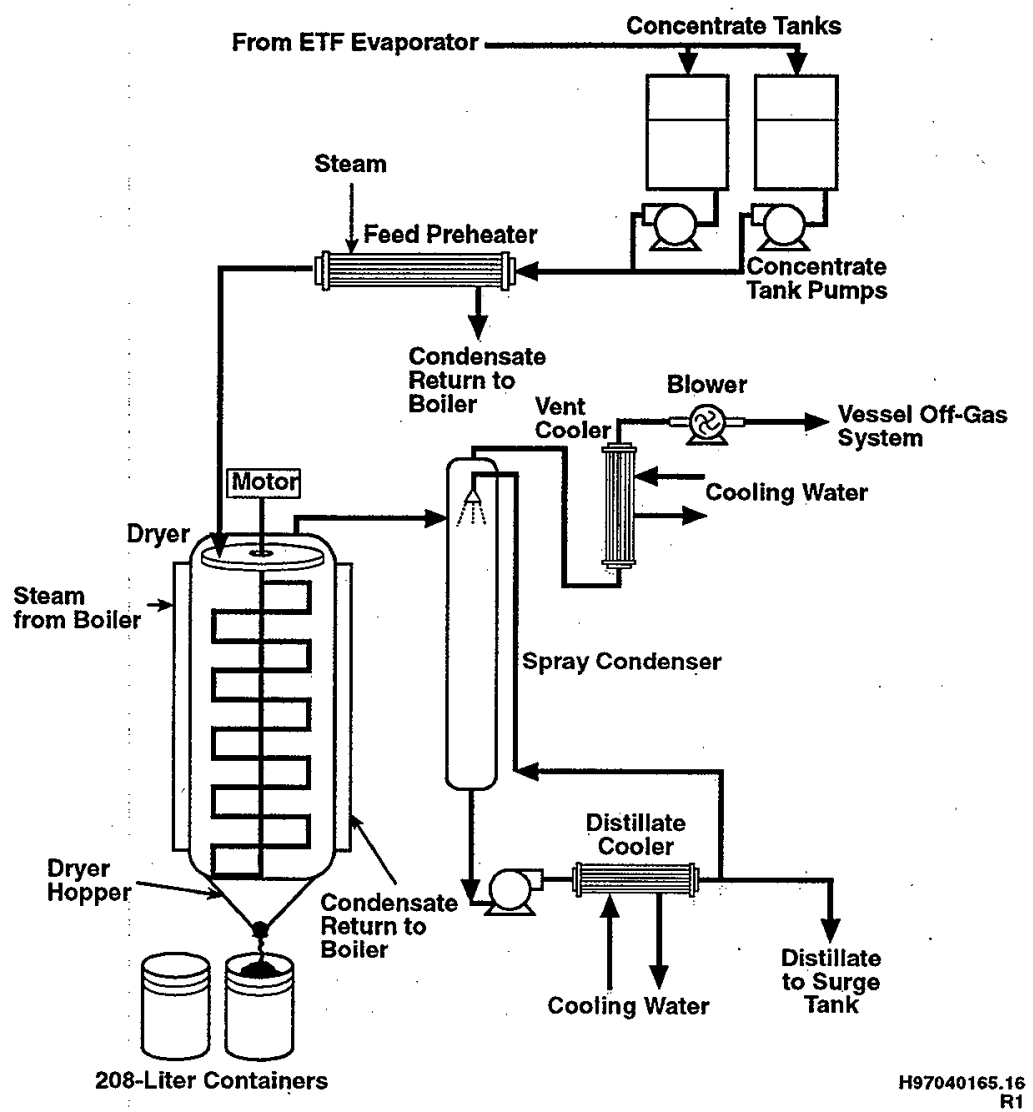

Figure 3-13. Thin Film Dryer. 


\subsection{TREATABLITY INFORMATION}

The treatability information presented in this delisting modification document follows a similar approach as the initial delisting petition and is based on previous laboratory and treatability testing, waste processing experience at the ETF, and vendor information. As in the initial delisting petition, the treatment efficiency is used to calculate a concentration envelope. When the characterization of a waste or batch of compatible waste, fall within the concentration envelope, treatment at the ETF will produce a nonhazardous, nondangerous waste that can be disposed pursuant to Washington State Waste Discharge Permit (ST4500) and the Final Delisting.

The difference between the initial up-front delisting petition and this modification is the track tecord and operating experience of the ETF. The ETF has treated over 174,000,000 liters of hazardous listed waste. This treated waste was disposed of at the SALDS in compliance with the Washington State Waste Discharge Permit ST 4500 and the Final Delisting.

To determine the treatability of the numerous potential leachate constituents (as determined in Section 2.2.2), the constituents were placed into groups having similar chemical properties. When actual operating or test data were not available for constituents to determine treatment efficiency, one or more constituents were selected to represent the treatability group. This treatability group concept was successfully used in the initial delisting petition (DOE/RL-92-72) and has been demonstrated through ETF operation.

This section identifies those potential leachate constituents that were not addressed in the Final Delisting; categorizes the constituents into the treatability groups; defines the waste concentration level where, following treatment the waste is no longer hazardous; and defines the treatment objectives for these constituents.

\subsection{CURRENT DELISTING LEVELS}

The current delisting levels (40 CFR 261, Appendix IX, Table 2) are included in Table 4-1. This table also lists other constituents examined in the initial delisting petition (DOE/RL-92-72) and the potential leachate constituents considered in this delisting modification. The assigned treatability group for each constituent also is included in Table 4-1.

The initial petition determined a constituent concentration envelope for which a waste could be treated at the ETF, based on one pass through the ETF, and meet health-based levels (HBLs). The current delisting levels are 10 times the HBLs. The factor of 10 is attributed to the applied groundwater attenuation factor.

\subsection{TREATABILITY GROUPS}

The initial delisting petition defined numerous chemical or treatability groups that were based on similar chemical structure and or physical properties. These same treatability groups are used in this delisting modification. The treatability groups are particularly important when determining the treatment efficiency of the many organic compounds included as potential leachate constituents. The organic constituents that have similar chemical structures exhibit comparable UV/OX destruction efficiencies. As an example, the volatile halogenated hydrocarbon group includes methyl chloride, methylene chloride, chloroform, and carbon tetrachloride. This example list of compounds is ordered with an increasing number of chloride atoms on the methane molecule and these all have similar treatment efficiencies in the ETF UV/OX treatment unit. 
These groups were devised to evaluate constituents based on testing performed using one or more constituents representing the group. This is a viable method to estimate the organic removal efficiency, without testing all constituents, because of the chemical similarities. The list of treatability groups is included in Table 4-2.

\subsection{CONSTITUENT TREATMENT EFFICIENCY}

The constituent treatment efficiency of the ETF is determined from actual waste processing at the ETF (Appendix A), previous treatability testing, and vendor information. The constituent treatment efficiency is used to back-calculate an ETF influent leachate constituent concentration that effectively can be treated during a single pass through ETF to meet required effluent concentration limits. These concentration limits are based on the HBL, as set forth by the EPA (EPA 1994), with a groundwater attenuation factor of 10 being applied. This approach is consistent with the requirements of the Final Delisting (40 CFR 261 Appendix IX, Table 2). This calculated influent leachate constituent concentration is defined as the concentration envelope, which is used to evaluate the treatability of an identified waste stream. However, because of the flexible design of the ETF, treated aqueous waste can be processed multiple times to ensure the treated waste is at or below the delisting level. It should be noted that some of the potential leachate constituents do not have a health-based level (HBL) established in the docket report. This situation is addressed in Sections 4.3.1 and 4.3.2.

Extensive treatability testing was performed as part of the initial delisting petition. This testing provided sufficient information to provide an up-front delisting before RCRA waste treatment at the ETF. An integrated pilot test was performed using UV/OX, RO, and IX. This testing was included in the initial delisting petition (DOE/RL-92-72).

The concentration envelope for the inorganic constituents is based on the treatability testing performed for the initial delisting petition (DOE/RL-92-72). The inorganic leachate constituents addressed by this delisting modification are sulfide, thallium, osmium, cobalt and tin. The inorganics are removed primarily by the RO and IX treatment operations in the ETF.

The concentration envelope for the organic constituents is based on ETF processing, prior treatability testing, and vendor information. With over 200 organic compounds included as potential leachate constituents in this delisting modification, a representative organic compound or compounds are used to represent the treatment efficiency for each organic treatability group. The organic compounds are removed primarily using the UV/OX treatment operation to destroy the organic compounds. The RO treatment step can remove, to some degree, organic compounds that have a molecular weight greater than 100 . However, for determining this concentration envelope, credit for removing organic compounds is given to the UV/OX unit.

\subsubsection{Inorganic Constituent Treatability Efficiency}

The inorganic constituents considered here are sulfide, thallium, osmium, cobalt and tin. Determining the maximum influent concentration envelope is straight forward for thallium, because thallium is the only one that has an established HBL. For the other inorganic constituents, a HBL of 1 milligram per liter was assumed so an example influent concentration envelope can be calculated. When a HBL is established for these inorganic constituents, the maximum influent concentration envelope to meet 10 times the $\mathrm{HBL}$ is determined by multiplying the newly determined $\mathrm{HBL}$ by the maximum influent concentration envelope as stated below. 
Sulfide was not considered in laboratory or treatability testing. However, sulfide is removed at the ETF by the strong oxidizing conditions resulting from hydrogen peroxide. Hydrogen peroxide is used primarily in the UV/OX system where extreme oxidizing conditions are present and also in the surge tank for biological control. The sulfide is readily converted to the sulfate ion. The sulfide removal efficiency is expected to be greater than 99.9 percent. Using the assumed HBL of 1 milligram per liter the maximum influent concentration envelope for sulfide to meet a treatment target of 10 times the HBL is 10,000 milligrams per liter.

Thallium, osmium, cobalt, and tin were not included in laboratory or treatability testing; however, based on pilot testing of other metals with similar valance states. The removal efficiency by the RO unit for thallium, osmium, cobalt, and tin is greater than 93.4 percent, greater than 97.5 percent, greater than 98.1 percent, and greater than 97.5 percent, respectively. Including a removal efficiency of 99 percent for IX treatment, the overall ETF treatment efficiency for these metals is greater than 99.9 percent. The HBL for thallium is 0.002 milligram per liter. The maximum influent concentration envelope for thallium to meet 10 times the HBL is 20 milligrams per liter. Using the assumed HBL of 1 milligram per liter for osmium, cobalt, and tin, the maximum influent concentration envelope to meet 10 times the $\mathrm{HBL}$ is 10,000 milligrams per liter.

\subsubsection{Organic Constituent Treatability Efficiency}

In the unlikely event that hazardous organic compounds are found in the leachate from F039 listed waste, the ETF must be able to treat these organics below the level where these threaten public health or the environment. This evaluation considers each potential leachate constituent. This organic constituent treatability analysis incorporates data from ETF aqueous waste processing, treatability testing, and vendor information. The Final Delisting specifies delisting levels for 23 organic potential leachate constituents. The initial delisting petition includes treatability results for an additional 18 organic potential leachate constituents. To finish out the list of organic potential leachate constituents, vendor information, treatability information and experience are used to determine the concentration envelope that can be treated at the ETF.

The ETF successfully has treated over $174,000,000$ liters of hazardous listed waste. The major organic compounds treated include acetone, 1-butanol, 2-butanone (also called methyl ethyl ketone), 2-butoxyethanol, carbon tetrachloride, tetrahydrofuran, and tributyl phosphate. Appendix A includes treatment efficiencies of constituents treated at the ETF and disposed at the SALDS.

A slight terminology difference is used here to express the treatment efficiency from that used in the initial delisting petition. In the initial delisting petition, the oxidation rate constant was calculated from observed test results based on the test configuration. The observed decrease in the organic compound concentration over time followed first order reaction kinetics. The test results were scaled up to the ETF UV/OX system. The scale-up parameters included the total ultraviolet energy, the hydrogen peroxide concentration, and ultraviolet energy per UV/OX reactor volume. New information provided from the vendor directly incorporates the scale-up parameters of volume and energy input with a first order reaction kinetics curve into a factor specific to the organic compound. This factor is based on an organic constituent concentration decrease of 10 times, 1 order of magnitude. The vendor calls the scale-up factor Electrical Energy per $\operatorname{Order}(\mathrm{EE} / \mathrm{O})$. The $\mathrm{EE} / \mathrm{O}$ is different for each organic compound; however, groups of compounds will tend to have similar EE/Os.

The UV/OX key design factor EE/O is defined as the UV light energy in terms of kilowatt hours of electricity required to reduce the concentration of a compound in 1,000 gallons by 1 order of magnitude (or 90 percent). The unit for $E E / O$ is kilowatt hour per 1,000 gallons/order. For example, if it takes 10 kilowatt hours of electricity to reduce the concentration of a target compound from 10 parts per million 
1 to 1 part per million ( 1 order of magnitude or 90 percent) in 1,000 gallons of aqueous waste, the $E E / 0$ is 10 kilowatt hours per 1,000 gallons per order for that compound. It would take another 10 kilowatt hours to reduce the compound from 1 part per million to 0.1 part per million.

The EE/O is determined through laboratory testing in the same way as the oxidation rate constant. The UV/OX vendor has supplied the EE/O for the organic constituents. The treatability group concept was used by the vendor for the EE/O of constituents not included in their extensive database. The lower the $\mathrm{EE} / \mathrm{O}$, the greater the treatment efficiency. The formula for $\mathrm{EE} / \mathrm{O}$ is as follows:

$$
\mathrm{EE} / \mathrm{O}=\mathrm{UV} \text { Dose } / \log \left(\mathrm{C}_{(\mathrm{i})} / \mathrm{C}_{(\mathfrak{l})}\right)
$$

Where:

The $\mathrm{UV}$ dose is $\mathrm{kWh} / 1,000$ gallons, the UV energy going into the water

$\mathrm{C}_{(\mathfrak{)})}$ is the final constituent concentration after treatment assumed to be the HBL $\times 10$. The groundwater attenuation factor is included.

$\mathrm{C}_{(i)}$ is the initial constituent concentration before treatment.

The design constraints of the ETF UV/OX system are 720 kilowatt electric (662.4 kilowatt UV energy, using a 92 percent conversion factor), 172 gallons per minute flow rate through the UV/OX equipment, and 2.08 minutes residence time in the UV/OX treatment unit. The design UV dose for the ETF UV/OX unit is 64.19 kilowatt hour per 1,000 gallons. The influent organic constituent concentration that can be treated in one pass through the UV/OX unit and reach the HBL times 10 limit is calculated as follows:

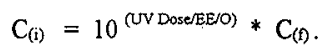

The influent organic constituent concentration that can be treated at the ETF is calculated according to equation (2). Several other considerations, as discussed in the following, were used to determine the envelope concentration. Table 4-3 presents the envelope concentrations. Appendix B includes the HBL and the solubility limits for each of the organic compounds.

Additional considerations were used to finalize the influent organic constituent concentration. These considerations include the LERF liner compatibility and the solubility limit of the organic compound. The primary LERF liner is a high-density polyethylene material, the same material as the liner used in the LLBG. Because some of the organic compounds are so effectively treated by UV/OX, the calculated influent concentration, using equation (2), is greater than the liner compatibility limits and/or greater than the solubility limit. When this situation is encountered, the lower of the liner compatibility limit or the solubility limit is used for the influent concentration. In some cases, there was no EPA established HBL for an organic compound. When this occurred, an HBL of 1 milligram per liter was assumed to complete the evaluation. When an $\mathrm{HBL}$ is established for the constituent, the maximum influent concentration envelope is determined by multiplying the newly determined HBL by the maximum influent concentration envelope value established for that constituent in Table 4-3. Appendix B includes the determination of the envelope along with the solubility and LERF liner compatibility considerations.

\subsection{STANDARDS TREATED WASTE MUST MEET}

The aqueous hazardous waste treated at ETF must meet discharge restrictions delineated in Washington State Waste Discharge Permit ST 4500 and the Final Delisting. The Washington State Waste Discharge Permit ST 4500 imposes daily maximum enforcement limits, average monthly enforcement limits, and 
average monthly early waming values. The Final Delisting imposes delisting limits. The limits for each parameter are listed in Table 4-4.

There are only 10 constituents identified in the waste designations of waste currently waiting disposal at the LLBG, refer to Chapter 2.0, Table 2-1, that fall into the category of difficult to treat in the ETF UV/OX unit. Of these 10 constituents, only 1,2-dichloroethane, carbon tetrachloride, and chloroform are listed as part of the waste designation in greater than one-tenth of one percent of the total waste volume. These three organic compounds have the highest probability of being in the leachate. All of the other organic compounds listed in Table 2-1 are associated with waste that individually would make up less than onetenth of one percent of the total waste volume. With Table 2-1 being a 'typical' distribution of waste to be disposed of in the LLBG, the total quantity of hard to treat organics that potentially would be in the leachate is very small and within the treatability envelope.

The influent concentration envelope, shown in Table 4-3, indicates the concentrations of hazardous waste that can be treated at the ETF in a once-through treatment process. The ETF design includes the capability of retreating (recycling) aqueous waste that does not meet the treatment limits. Recycling the contents of a verification tank for further treatment before disposal, shown in Figure 3-11, allows for a flexible influent concentration envelope. This flexibility provides the ETF with the ability to treat a wide variety of aqueous wastes containing a broad spectrum of hazardous constituents and still meet the treatment limits before disposal. 
This page intentionally left blank. 
Table 4-1. Constituents Delisted and Considered in Delisting Modification.

\begin{tabular}{|c|c|c|c|c|c|c|}
\hline $\begin{array}{l}\text { Treatability } \\
\text { Group }\end{array}$ & Constituent & CAS no. & Waste Number & $\begin{array}{c}\text { Delisting } \\
\text { Level (ppm) }\end{array}$ & \begin{tabular}{|c|} 
Considered in \\
Initial \\
Delisting \\
Petition \\
\end{tabular} & $\begin{array}{c}\text { Considered in } \\
\text { Modified } \\
\text { Delisting }\end{array}$ \\
\hline 1 & 2,4-Dimethylphenol & $105-67-9$ & U101 & & & $x$ \\
\hline 1 & Coal tar creosote & $8007-45-2$ & & & 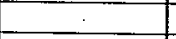 & $x$ \\
\hline 1 & Creosote & $8001-58-9$ & & & & $\underline{x}$ \\
\hline 1 & Cresol [Cresylic acid] & $1319-77-3$ & F001-5, U052 & 20 & $t$ & \\
\hline 1 & m-Cresol [3-Methylphenol] & 108-39-4 & F001-5, U052 & & & $x$ \\
\hline 1 & o-Cresol [2-Methylphenol] & $95-48-7$ & F001-5, U052 & & & $x$ \\
\hline 1 & p-Cresol [4-Methylphenol] & $106-44-5$ & F001-5, U052 & & & $x$ \\
\hline 1 & Phenol & $108-95-2$ & U188 & & $t$ & \\
\hline 2 & 2,3,4,6-Tetrachlorophenol & $58-90-2$ & F020-23, F026-28 & & & $x$ \\
\hline 2 & $\begin{array}{l}\text { 2,3,4,6-tetrachlorophenol, potassium } \\
\text { salt }[2,3,4,6 \text {-tetrachlorophenol salt] }\end{array}$ & $53535-27-6$ & & & & $x$ \\
\hline 2 & $\begin{array}{l}\text { 2,3,4,6-tetrachlorophenol, sodium sait } \\
{[2,3,4,6 \text {-tetrachlorophenol, salt] }}\end{array}$ & $25567-55-9$ & . & & & $\mathrm{x}$ \\
\hline 2 & 2,4,5-Trichlorophenol & $95-95-4$ & $\mathrm{~F} 020-23, \mathrm{~F} 026-28$ & & & $\mathrm{x}$ \\
\hline 2 & 2,4,6-Tribromophenol & $118-79-6$ & & & & $\mathrm{x}$ \\
\hline 2 & 2,4,6-Trichlorophenol & $88-06-2$ & $\mathrm{~F} 020-23, \mathrm{~F} 026-28$ & & & $\mathrm{x}$ \\
\hline 2 & 2,4-Dichlorophenol & $120-83-2$ & U081 & & & $x$ \\
\hline 2 & 2,6-Dichlorophenol & $87-65-0$ & U082 & & & $\underline{x}$ \\
\hline 2 & m-Chloro-3-methylphenol & $59-50-7$ & U039 & & . & $x$ \\
\hline 2 & o-Chlorophenol & $95-57-8$ & U048 & & & $x$ \\
\hline 2 & Pentachlorophenol & $87-86-5$ & $\mathrm{F020-23,F026-28}$ & & $t$ & \\
\hline 2 & $\begin{array}{l}\text { Potassium pentachlorophenate } \\
\text { [Pentachlorophenol salt] }\end{array}$ & $7778-73-6$ & & & & $x$ \\
\hline 2 & $\begin{array}{l}\text { Sodium pentachlorophenate } \\
\text { [Pentachlorophenol salt] }\end{array}$ & $131-52-2$ & & & & $x$ \\
\hline 3 & Acenaphthene & $83-32-9$ & F039 & & & $x$ \\
\hline 3 & Acenaphthylene & $208-96-8$ & F039 & & & $x$ \\
\hline 3 & Anthracene & 120-12-7 & F039 & & & $x$ \\
\hline 3 & Benzene & $71-43-2$ & F001-5, U019 & 0.05 & $t$ & \\
\hline 3 & Benzenearsonic acid (Arsenic) & $98-05-5$ & & & & $x$ \\
\hline
\end{tabular}


Table 4-1. Constituents Delisted and Considered in Delisting Modification.

\begin{tabular}{|c|c|c|c|c|c|c|}
\hline $\begin{array}{l}\text { Treatability } \\
\text { Group } \\
\text {.... }\end{array}$ & 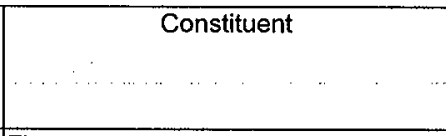 & 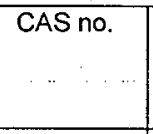 & Waste Number & $\begin{array}{c}\text { Delisting } \\
\text { Level (ppm) }\end{array}$ & \begin{tabular}{|c|}
$\begin{array}{c}\text { Considered in } \\
\text { Initial } \\
\text { Delisting } \\
\text { Petition }\end{array}$ \\
\end{tabular} & $\begin{array}{l}\text { Considered in } \\
\text { Modified } \\
\text { Delisting }\end{array}$ \\
\hline 3 & Fluorene & $86-73-7$ & F039 & & & $x$ \\
\hline 3 & Naphthalene & $91-20-3$ & U051, U165 & 10 & $t$ & \\
\hline 3 & Phenanthrene & $85-01-8$ & U051 & & & $x$ \\
\hline 3 & Styrene & $100-42-5$ & & & & $x$ \\
\hline 4 & 3-Methylcholanthrene & $56-49-5$ & U157 & & & $x$ \\
\hline 4 & Benzo(a)anthracene & $56-55-3$ & U018 & & & $x$ \\
\hline 4. & Benzo(a)pyrene & $50-32-8$ & U022 & & & $x$ \\
\hline 4 & Benzo(b)fluoranthene & 205-99-2 & F039 & & & $\mathbf{x}$ \\
\hline 4 & Benzo(ghi)perylene & 191-24-2 & F039 & & & $x$ \\
\hline 4 & Benzo(k)fluoranthene & $207-08-9$ & F039 & & & $x$ \\
\hline 4 & Chrysene & 218-01-9 & U050 & & & $x$ \\
\hline 4 & Dibenzo[a,e]pyrene & $192-65-4$ & F039 & & & $x$ \\
\hline 4 & Dibenzo[a,h]anthracene & $53-70-3$ & U063 & & & $x$ \\
\hline 4 & Dibenzo[a,h]pyrene & 189-64-0 & & & & $x$ \\
\hline 4 & Fluoranthrene & 206-44-0 & U120 & & & $x$ \\
\hline 4 & Indeno $(1,2,3, \mathrm{~cd})$ pyrene & $193-39-5$ & F039 & & & $x$ \\
\hline 4 & Pyrene & 129-00-0 & U051 & & $t$ & \\
\hline $5 \mid \mathbf{a}$ & 1,2,4,5-Tetrachlorobenzene & 95-94-3 & U207 & & & $\mathrm{x}$ \\
\hline $5 \sqrt{a}$ & 1,2,4-Trichlorobenzene & $120-82-1$ & F039 & & & $x$ \\
\hline 5 & m-Dichlorobenzene & $541-73-1$ & F001-5, U071 & & & $\mathrm{x}$ \\
\hline 5. & o-Dichlorobenzene & $95-50-1$ & $\mathrm{U} 070$ & & & $x$ \\
\hline 5 & p-Dichlorobenzene & 106-46-7 & U072 & 0.75 & $t$ & \\
\hline 5 a & Pentachlorobenzene & $608-93-5$ & U183 & & & $x$ \\
\hline 5 & $\begin{array}{l}\text { 2-Chloronaphthalene [beta- } \\
\text { Chloronaphthalene] }\end{array}$ & $91-58-7$ & U047 & & & $x$ \\
\hline 5 & Heptachlorodibenzofuran & $38998-75-3$ & & & & $\mathrm{x}$ \\
\hline 5 & Heptachlorodibenzo-p-dioxins & $35822-46-9$ & & & & $\mathrm{x}$ \\
\hline 6 a & Hexachloroethane. & $67-72-1$ & U131 & 0.06 & $t$ & \\
\hline $6 \mathrm{~b}$ & 1,3-Dichloropropene & $542-75-6$ & U084 & & & $x$ \\
\hline $6 \mid \mathbf{b}$ & Chloroprene [2-Chloro-1,3-butadiene] & $126-99-8$ & F039 & & & $x$ \\
\hline
\end{tabular}


Table 4-1. Constituents Delisted and Considered in Delisting Modification.

\begin{tabular}{|c|c|c|c|c|c|c|}
\hline $\begin{array}{l}\text { Treatability } \\
\text { Group }\end{array}$ & Constituent & CAS no. & Waste Number & $\begin{array}{c}\text { Delisting } \\
\text { Level }(\mathrm{ppm})\end{array}$ & \begin{tabular}{|c|} 
Considered in \\
Initial \\
Delisting \\
Petition \\
\end{tabular} & $\begin{array}{l}\text { Considered in } \\
\text { Modified } \\
\text { Delisting }\end{array}$ \\
\hline \begin{tabular}{l|l}
6 & $b$ \\
\end{tabular} & cis-1,3-Dichloropropene & $10061-01-5$ & U084 & & & $x$ \\
\hline $6 \mid b$ & Hexachlorobutadiene & $87-68-3$ & U128 & & & $\mathbf{x}$ \\
\hline $6 \mid b$ & Hexachloropropene & $1888-71-7$ & U243 & & 5 & $x$ \\
\hline $7 \mid a$ & bis(2-Chloroethoxy) methane & $111-91-1$ & U024 & & & $x$ \\
\hline $7 \mid \mathbf{a}$ & Bis(2-Chloroethyl) ether & $111-44-4$ & U025 & & $t$ & \\
\hline 7 a & Bis (2-Chloroisopropyl) ether & $39638-32-9$ & U027 & & & $x$ \\
\hline $7 \mid \mathbf{a}$ & Dichloroisopropyl ether & $108-60-1$ & U027 & & & $x$ \\
\hline $7 / b$ & 4-Bromophenylphenyl ether & $101-55-3$ & U030 & & & $x$ \\
\hline $7 \mathrm{~b}$ & 4-Chlorophenyl phenyl ether & $7005-72-3$ & & & $\mathrm{t}$ & \\
\hline 8 & Bis(2-Ethylhexyl) phthalate & $117-81-7$ & U028 & & $t$ & \\
\hline 8 & Butylbenzylphthalate & $85-68-7$ & F039 & & & $x$ \\
\hline 8 & Diethylphthalate & $84-66-2$ & U088 & & & $x$ \\
\hline 8 & Dimethyl phthalate & $131-11-3$ & U102 & & & $x$ \\
\hline 8 & Di-n-butylphthalate & $84-74-2$ & U069 & & & $x$ \\
\hline 8 & Di-n-octylphthalate & $117-84-0$ & U107 & 7 & & \\
\hline $9 \mathrm{a}$ & 1-Butanol & $71-36-3$ & F001-5, U031 & 40 & $\mathrm{t}$ & \\
\hline 99 & Benzyl alcohol & $100-51-6$ & & 100 & $t$ & \\
\hline 9 a & Isobutyl alcohol & $78-83-1$ & F001-5, U140 & & & $x$ \\
\hline $9 \sqrt{a}$ & Methanol & $67-56-1$ & F001-5, U154 & & & $x$ \\
\hline 9 & 1,4-Dioxane [1,4-Diethyleneoxide] & $123-91-1$ & U108 & & & $x$ \\
\hline 9 & $2,4,5-T$ & $93-76-5$ & F039 & & & $x$ \\
\hline 9 & 2,4,5-TP [Silvex] & $93-72-1$ & [D017] & & & $x$ \\
\hline 9 & $\begin{array}{l}\text { 2,4-D [2,4-Dichlorophenoxyacetic } \\
\text { acid] }\end{array}$ & $94-75-7$ & $\mathrm{U} 240$ & & & $x$ \\
\hline 9 & Carbofuran phenol & 1563-38-8 & U367 & & & $\mathrm{x}$ \\
\hline 9 & Ethyl Acetate & $141-78-6$ & F039 & & & $x$ \\
\hline 9 & Kepone & $143-50-0$ & U142 & & & $x$ \\
\hline 9 & Lead subacetate (Lead) & $1335-32-6$ & & & & $x$ \\
\hline 9 & Methyl methacrylate & $80-62-6$ & U162 & & & $x$ \\
\hline 9 & Methyl methanesulfonate & $66-27-3$ & F039 & & & $x$ \\
\hline
\end{tabular}


Table 4-1. Constituents Delisted and Considered in Delisting Modification.

\begin{tabular}{|c|c|c|c|c|c|c|c|}
\hline $\begin{array}{l}\text { Treatabilit } \\
\text { Group }\end{array}$ & ity & \begin{tabular}{|llll}
\multicolumn{4}{|c|}{ Constituent } \\
\\
$\ldots \ldots$ & $\ldots$ & $\ldots$ & \\
\end{tabular} & $\begin{array}{ll}\text { CAS no. } & \\
& \\
& \end{array}$ & $\begin{array}{c}\text { Waste Number } \\
\begin{array}{ccc} & & \\
\end{array}\end{array}$ & $\begin{array}{c}\text { Delisting } \\
\text { Level (ppm) }\end{array}$ & $\begin{array}{c}\text { Considered in } \\
\text { Initial } \\
\text { Delisting } \\
\text { Petition } \\
\end{array}$ & $\begin{array}{l}\text { Considered in } \\
\text { Modified } \\
\text { Delisting }\end{array}$ \\
\hline 9) & & Phenyl mercuric acetate & $62-38-4$ & P092 & & & $x$ \\
\hline 9 & & Phthalic anhydride & $85-44-9$ & $\cup 190$ & & & $x$ \\
\hline 9 & & Safrole & $94-59-7$ & $\cup 203$ & & & $x$ \\
\hline 9 & & Vinyl acetate & $108-05-4$ & & & & $x$ \\
\hline 10 a & a & 2-Acethylaminofluorene & $53-96-3$ & U005 & & & $\mathrm{x}$ \\
\hline 10 a & $a$ & 4-Aminobiphenyl & $92-67-1$ & F039 & & & $x$ \\
\hline $10 a$ & a & Diphenylamine & $122-39-4$ & $\mathrm{FO} 39$ & & & $x$ \\
\hline 10]a & $a$ & o-Phenylenediamine & $95-54-5$ & & . & & $x$ \\
\hline $10 \mid a$ & a & Phenacetin [p-Acetophenetidide] & $62-44-2$ & U187 & & & $\mathrm{x}$ \\
\hline 10$] a$ & $a$ & p-Phenylenediamine & $106-50-3$ & & $=$ & & $x$ \\
\hline $10: a$ & $a$ & Selenourea (Selenium) & $630-10-4$ & & & & $\mathrm{x}$ \\
\hline $10 \mid a$ & a & Toluene-2,4-diamine & $95-80-7$ & & & & $x$ \\
\hline $10 \mathrm{~b}$ & b & 4,4'-Methylenebis(2-chloroaniline) & $101-14-4$ & U158 & & & $x$ \\
\hline $10 \mathrm{~b}$ & b & Aniline & $62-53-3$ & U012 & & $t$ & \\
\hline $10 \mathrm{~b}$ & b & o-Nitroaniline & $88-74-4$ & & & & $x$ \\
\hline $10 \mathrm{~b}$ & $\mathbf{b}$ & p-Chloroaniline & $106-47-8$ & $\mathrm{P024}$ & & . & $x$ \\
\hline $10 \mid \mathrm{b}$ & $\mathbf{b}$ & p-Nitroaniline & $100-01-6$ & & & & $\mathrm{x}$ \\
\hline $10 . \mathrm{c}$ & c & Acetonitrile & $75-05-8$ & $\mathrm{U003}$ & & $t$ & \\
\hline $10 . \mathrm{c}$ & c & Acrylonitrile & $107-13-1$ & U.009 & & & $\mathrm{x}$ \\
\hline $10 \mid c$ & c & Methacrylonitrile & $126-98-7$ & U152 & 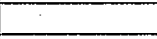 & & $\mathrm{x}$ \\
\hline $10 \mathrm{~d}$ & d & 1,3-Dinitrobenzene & $99-65-0$ & & & & $\mathrm{x}$ \\
\hline $10 \mathrm{~d}$ & $d$ & 2,4-Dinitrophenol & $51-28-5$ & P048 & & & $\mathrm{x}$ \\
\hline $10 \mathrm{~d}$ & d & 2,4-Dinitrotoluene & $121-14-2$ & U105 & & & $x$ \\
\hline $10 \mathrm{~d}$ & d & 2,6-Dinitrotoluene & $606-20-2$ & U106 & & & $x$ \\
\hline $10 \mathrm{~d}$ & d & Nitrobenzene & 98-95-3 & F001-5, U169 & & $\mathbf{t}$ & \\
\hline $10 \mathrm{~d}$ & d & Pentachloronitrobenzene (PCNB) & $82-68-8$ & U185 & & & $\mathrm{x}$ \\
\hline
\end{tabular}


Table 4-1. Constituents Delisted and Considered in Delisting Modification.

\begin{tabular}{|c|c|c|c|c|c|c|}
\hline $\begin{array}{l}\text { Treatability } \\
\text { Group }\end{array}$ & Constituent & CAS no. & Waste Number & $\begin{array}{c}\text { Delisting } \\
\text { Level (ppm) }\end{array}$ & \begin{tabular}{|c|} 
Considered in \\
Initial \\
Delisting \\
Petition \\
\end{tabular} & $\begin{array}{l}\text { Considered in } \\
\text { Modified } \\
\text { Delisting }\end{array}$ \\
\hline $10 \mathrm{~d}$ & $p$-Nitrophenol & $100-02-7$ & U170 & & & $x$ \\
\hline $10 \mathrm{e}$ & Dibenz $[a, h]$ acridine & $226-36-8$ & & & & $x$ \\
\hline $10 \mathrm{e}$ & Dibenz $[a$,j]acridine & 224-42-0 & & $\vdots$ & & $x$ \\
\hline $10 e$ & $\mathrm{~N}$-Nitrosodiethylamine & $55-18-5$ & U174 & & & $x$ \\
\hline $10 \mathrm{e}$ & N-Nitrosodimethylamine & $62-75-9$ & P082 & & $t$ & \\
\hline $10 \mathrm{e}$ & N-Nitrosodi-n-butylamine & $924-16-3$ & U172 & & & $x$ \\
\hline $10 \mathrm{e}$ & N-Nitroso-di-n-dipropylamine & $621-64-7$ & U111 & & $\mathrm{t}$ & \\
\hline $10 \mathrm{e}$ & N-Nitrosodiphenylamine & $86-30-6$ & F039 & & & $x$ \\
\hline $10 \mathrm{e}$ & N-Nitrosomethylethylamine & $10595-95-6$ & F039 & & & $x$ \\
\hline $10 \mathrm{e}$ & N-Nitrosomorpholine & $59-89-2$ & F039 & & & $x$ \\
\hline $10 \mathrm{e}$ & N-Nitrosopiperidine & $100-75-4$ & U179 & & & $x$ \\
\hline $10 / \mathrm{e}$ & N-Nitrosopyrrolidine & $930-55-2$ & U180 & & & $x$ \\
\hline $10 \mathrm{f}$ & Methapyrilene & $91-80-5$ & U155 & & & $x$ \\
\hline $10 \mathrm{f}$ & Pyridine & 110-86-1 & F001-5, U196 & & $t$ & \\
\hline 10 & Aldicarb sulfone & 1646-88-4 & P203 & & & $x$ \\
\hline 10 & Disulfiram & $97-77-8$ & & & & $x$ \\
\hline 10 & Manganese dimethyldithiocarbamate & 15339-36-3 & P196 & & & $x$ \\
\hline 10 & Physostigmine & $57-47-6$ & $\mathrm{P} 204$ & & & $\mathrm{x}$ \\
\hline 10 & Physostigmine salicylate & $57-64-7$ & P188 & & & $x$ \\
\hline 10 & $\begin{array}{l}\text { Potassium n-hyroxymethyl-n-methyl- } \\
\text { dithiocarbamate }\end{array}$ & $51026-28-9$ & & & & $x$ \\
\hline 10 & Sodium diethyldithiocarbamate & $148-18-5$ & & & & $x$ \\
\hline 10 & Tetrabutylthiuram disulfide & $1634-02-2$ & & & & $x$ \\
\hline 11 & A2213 & $30558-43-1$ & U394 & & & $x$ \\
\hline 11 & Aldrin & $309-00-2$ & P004 & & & $x$ \\
\hline 11 & Alpha-BHC & $319-84-6$ & U129 & & & $x$ \\
\hline 11 & Aramite & $140-57-8$ & F039 & & & $x$ \\
\hline 11 & Barban & $101-27-9$ & U280 & & & $x$ \\
\hline 11 & Bendiocarb & $22781-23-3$ & U278 & & & $x$ \\
\hline 11 & Benomyl & $17804-35-2$ & U271 & & & $x$ \\
\hline
\end{tabular}




\begin{tabular}{|c|c|c|c|c|c|c|c|c|c|c|c|c|c|c|c|c|c|c|c|c|c|c|c|c|}
\hline 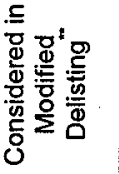 & $x$ & $x$ & $x$ & $\mathbf{x}$ & $x$ & $x \mid x$ & $x \mid x$ & $x \mid x$ & $x$ & $x$ & $\times$ & $x \mid>$ & $x \times$ & $x$ & $\mathbf{x}$ & $\times \mid x$ & $\times$ & $x \mid x$ & $|x|$ & $x \mid x$ & $\mathbf{x}$ & $x$ & $\times>$ & $\times$ \\
\hline 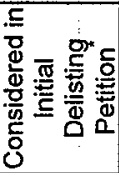 & & & & & & & & & & & & & & & & & & & & & 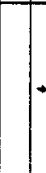 & & & \\
\hline
\end{tabular}

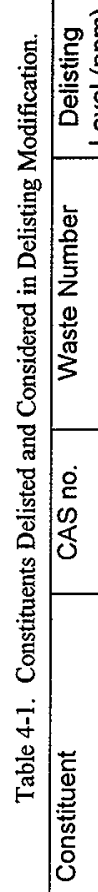

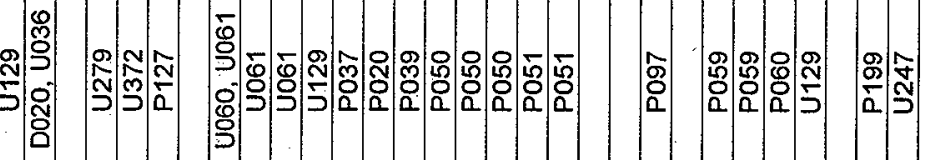

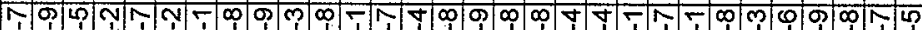
ம் ப் ம் ம் $\infty N$ 寸 ๙ लू

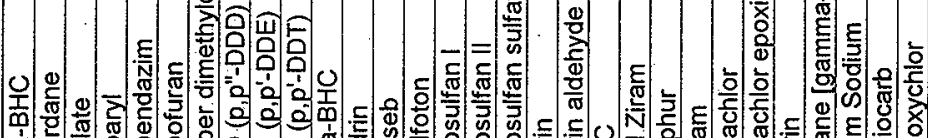

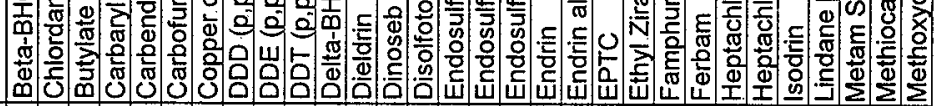


DOE/RL-98-62, Rev. 0

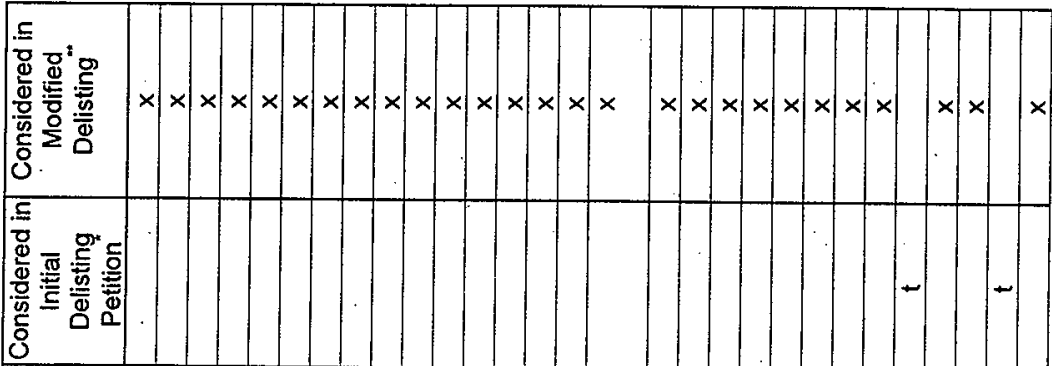

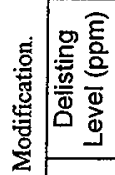

.

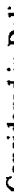

马

蛋

o

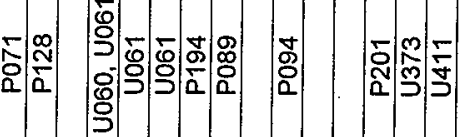

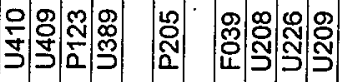

3

O-

ธ่์

จุ

(

N

Tन्म

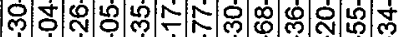

ó

ल స

$-6$

నั

त.

莺
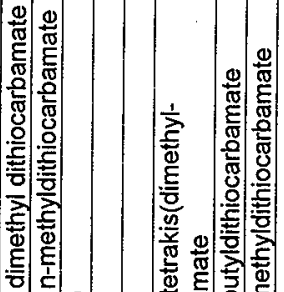

$\stackrel{0}{\square}$

톨

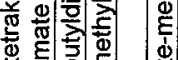

产

다.

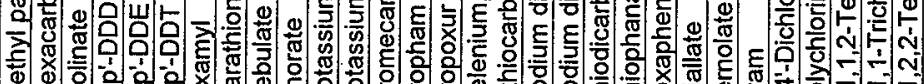

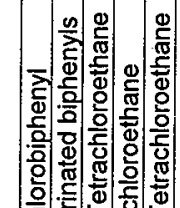
$\sum \sum \sum \sum$ O

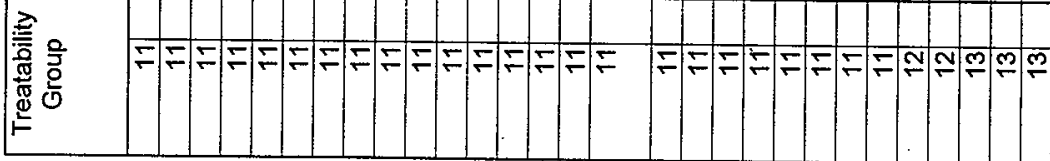



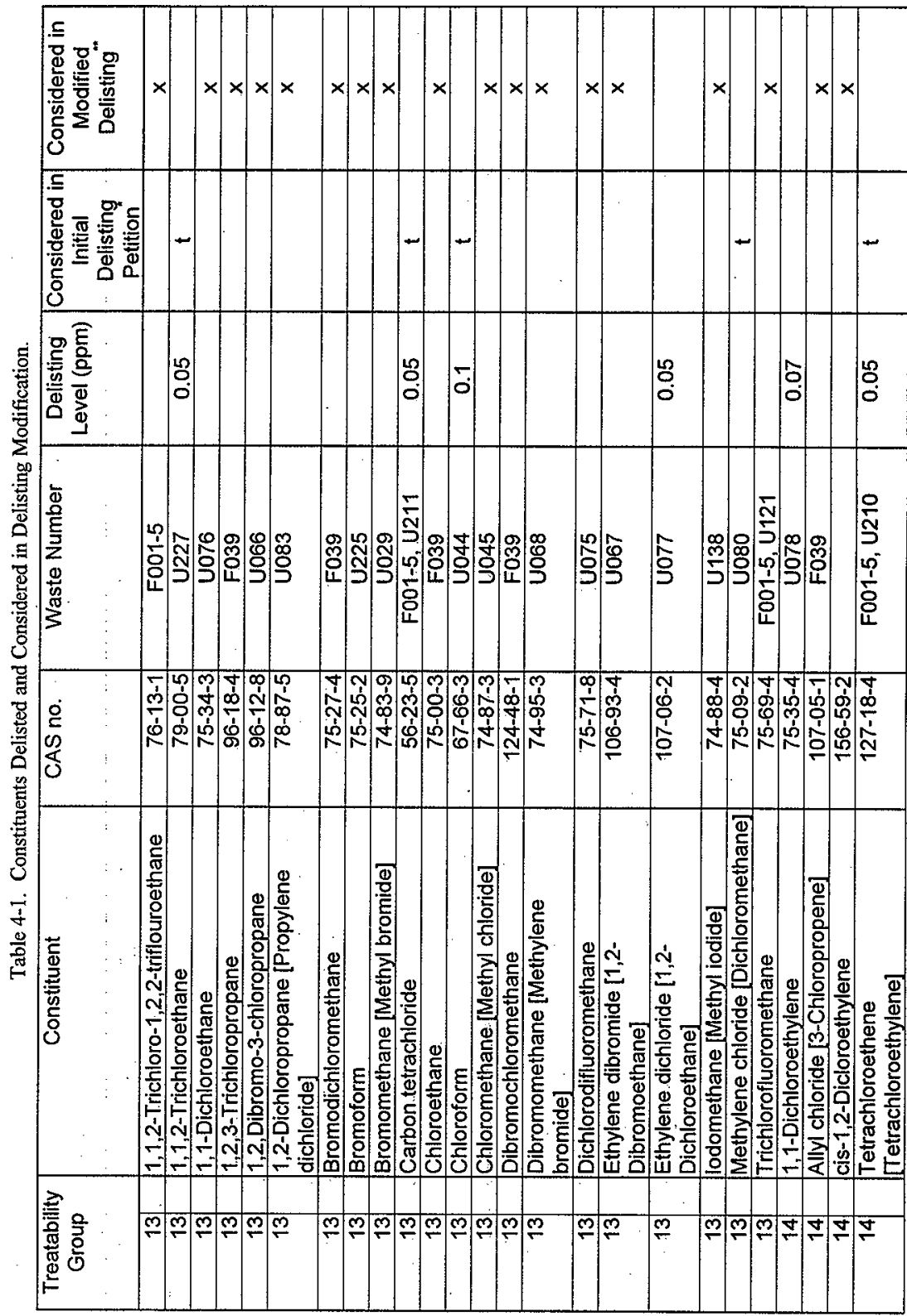
Table 4-1. Constituents Delisted and Considered in Delisting Modification.

\begin{tabular}{|c|c|c|c|c|c|c|}
\hline $\begin{array}{l}\text { Treatability } \\
\text { Group }\end{array}$ & Constituent & CAS no. & Waste Number & $\begin{array}{c}\text { Delisting } \\
\text { Level (ppm) }\end{array}$ & $\begin{array}{c}\text { Considered in } \\
\text { Initial } \\
\text { Delisting } \\
\text { Petition } \\
\end{array}$ & $\begin{array}{l}\text { Considered in } \\
\text { Modified } \\
\text { Delisting }\end{array}$ \\
\hline 14 & trans-1,2-Dichloroethylene & $156-60-5$ & U079 & & & $\mathrm{x}$ \\
\hline 14 & trans-1,3-Dichloropropene & $10061-02-6$ & U084 & & & $x$ \\
\hline 14 & Trichloroethylene & $79-01-6$ & F001-5, U228 & 0.05 & & \\
\hline 14 & Vinyl chloride & $75-01-4$ & U043 & 0.02 & & \\
\hline $15 \mid a$ & Ethylbenzene & $100-41-4$ & F001-5 & & & $x$ \\
\hline 15 a & Toluene & $108-88-3$ & F001-5, U051, U220 & 10 & $\mathrm{t}$ & \\
\hline 15 a & Xylenes (total) & $1330-20-7$ & U051, U239 & & & $x$ \\
\hline 16 & Chlorobenzene & 108-90-7 & F001-5, U037 & 1 & & \\
\hline 16. & Dichlorophenylarsine (Arsenic) & 696-28-6 & & & & $x$ \\
\hline $17 \sqrt[a]{a}$ & Acrolein & $107-02-8$ & P003 & & $\mathrm{t}$ & \\
\hline $18 \mathrm{a}$ & Isosafrole & $120-58-1$ & U141 & & & $x$ \\
\hline 18 & Ethyl ether & $60-29-7$ & F001-5, U117 & & & $x$ \\
\hline 18 & Ethyl methacrylate & $97-63-2$ & $\mathrm{U} 118$ & & & $x$ \\
\hline 18 & Tetrahydrofuran & $109-99-9$ & & & $t$ & \\
\hline 19 & Acetone & $67-64-1$ & F001-5, U002 & 40 & $t$ & \\
\hline 19 & Acetophenone & $98-86-2$ & U004 & & & $x$ \\
\hline 19 & Cyclohexanone & $108-94-1$ & F001-5, U057 & & & $x$ \\
\hline 19 & Methyl ethyl ketone [2-Butanone] & $78-93-3$ & F001-5, U159 & 200 & $t$ & \\
\hline 19 & $\begin{array}{l}\text { Methyl isobutyl ketone [2-Methyl-4- } \\
\text { pentanone] }\end{array}$ & $108-10-1$ & F001-5, U161 & 30 & $t$ & \\
\hline 20 & Carbon disulfide & $75-15-0$ & $\mathrm{~F} 001-5, \mathrm{P} 022$ & & & $x$ \\
\hline 20 & tris(2,3-Dibromopropyl) phosphate & $126-72-7$ & U235 & & & $x$ \\
\hline 21 & Aluminum & $7429-90-5$ & & & $t$ & \\
\hline 21 & Antimony & $7440-36-0$ & F039 & 0.06 & & \\
\hline 21 & Barium & $7440-39-3$ & P013 & 20 & $t$ & \\
\hline 21 & Beryllium & $7440-41-7$ & P015 & 0.04 & $t$ & \\
\hline 21 & Cadmium & $7440-43-9$ & [D006] & 0.05 & $t$ & \\
\hline 21 & Cesium & $7440-46-2$ & & & $t$ & \\
\hline 21 & Chromium & $7440-47-3$ & U032 & 1 & $t$ & \\
\hline 21 & Cobalt & $7440-48-4$ & & & & $x$ \\
\hline
\end{tabular}



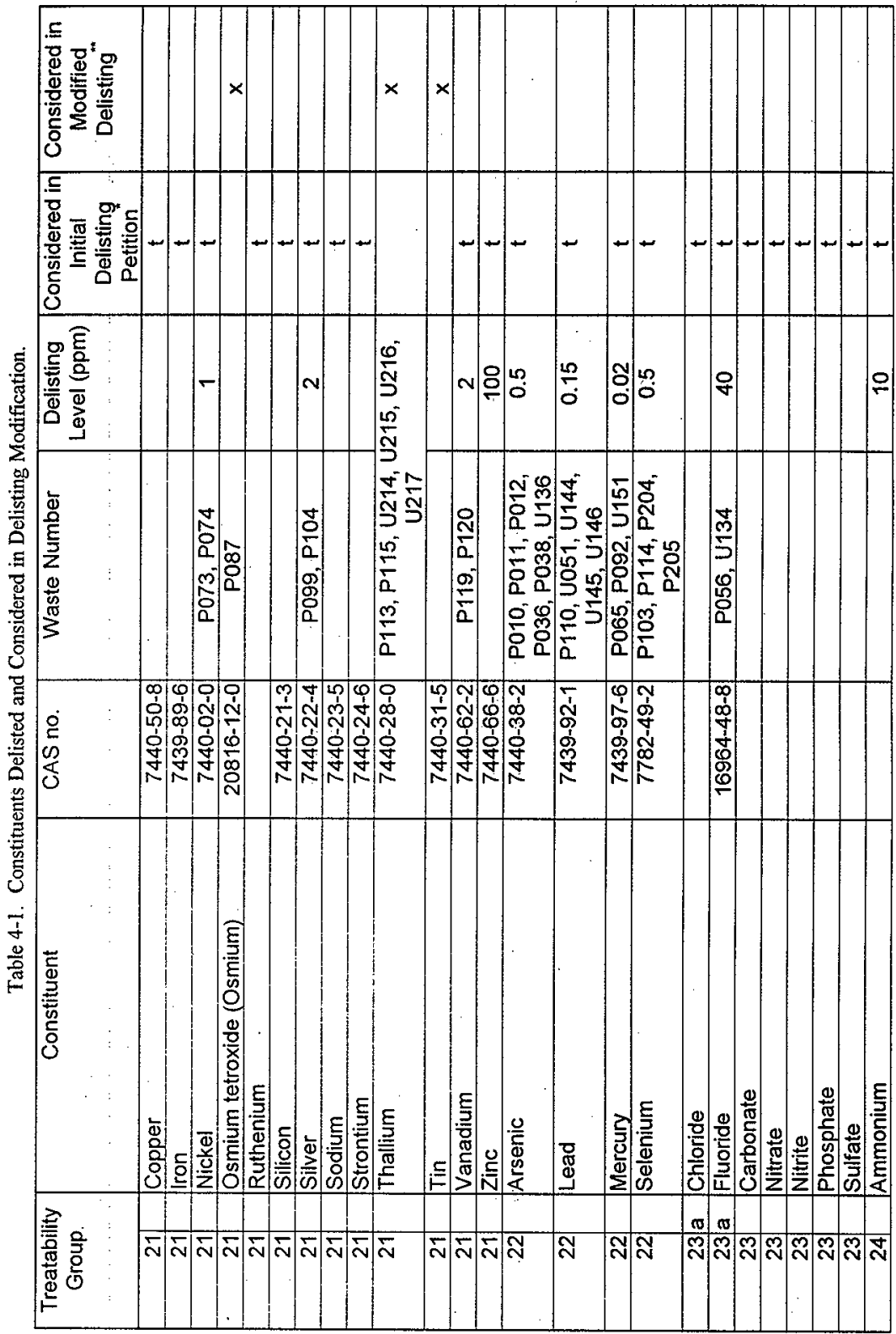
Table 4-1. Constituents Delisted and Considered in Delisting Modification.

\begin{tabular}{|c|c|c|c|c|c|c|}
\hline $\begin{array}{c}\text { Treatability } \\
\text { Group }\end{array}$ & Constituent & CAS no. & Waste Number & $\begin{array}{c}\text { Delisting } \\
\text { Level (ppm) }\end{array}$ & \begin{tabular}{|c|} 
Considered in \\
Initial \\
Delisting \\
Petition \\
\end{tabular} & $\begin{array}{l}\text { Considered in } \\
\text { Modified } \\
\text { Delisting }\end{array}$ \\
\hline 24 & Cyanide & $57-12-5$ & $\begin{array}{c}\text { P013, P021, P029, } \\
\text { P030, P063, P074, } \\
\text { P098, P099, P014, } \\
\text { P106, P121 }\end{array}$ & 2 & $t$ & \\
\hline 24 & Mercury fulminate [Mercury cyanate] & $628-86-4$ & & & 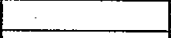 & 1 \\
\hline 24 & Sulfide & $8496-25-8$ & F039 & & & $x$ \\
\hline $25 a$ & $0,0,0-$ Triethyl phosphorothioate & 126-68-1 & & & & $x$ \\
\hline $25 \mid a$ & Tributyl phosphate & $126-73-8$ & & 0.2 & $t$ & \\
\hline $25 \mathrm{~b}$ & Tridecane & $629-50-5$ & & & $t$ & \\
\hline 25 & Isophorone & 7778-73-6 & & & & $x$ \\
\hline 25 & Tetraethyl lead (Lead) & $78-00-2$ & & & & $\mathrm{x}$ \\
\hline
\end{tabular}

* $t$ indicates constituents tested and reported as part of the initial delisting petition.

** $x=$ indicates the constituent considered in this delisting modification.

$1=$ this compound is a cyanate and will not be considered further because there is a delisting level for both mercury and cyanide.

CAS no. = Chemical Abstract Service number.

ppm = parts per million. 
Table 4-2. Treatability Groups.

\begin{tabular}{|c|c|}
\hline Group Name & Group No.* \\
\hline Phenols & 1 \\
\hline Substituted phenols & 2 \\
\hline Low molecular weight aromatics & 3 \\
\hline High molecular weight PAHs & 4 \\
\hline Chlorinated aromatic hydrocarbons & 5 \\
\hline Chlorinated benzenes & $5 a$ \\
\hline Chlorinated aiphatic hydrocarbons & 6 \\
\hline Halogenated alkanes & $6 \mathrm{a}$ \\
\hline Halogenated alkenes & $6 b$ \\
\hline Halogenated ethers & 7 \\
\hline Halogenated alkyl ethers & $7 \mathrm{a}$ \\
\hline Halogenated aryl ethers & $7 \mathrm{~b}$ \\
\hline Phthalates & 8 \\
\hline Miscellaneous oxygenated compounds & 9 \\
\hline Alcohol & $9 \mathrm{a}$ \\
\hline Organonitrogen compounds & 10 \\
\hline Amines & $10 \mathrm{a}$ \\
\hline Nitriles & $10 \mathrm{~b}$ \\
\hline Anilines & $10 \mathrm{c}$ \\
\hline Nitroaromatics & $10 \mathrm{~d}$ \\
\hline Nitrosoamines & $10 \mathrm{~d}$ \\
\hline Pyridines & $10 \mathrm{f}$ \\
\hline Pesticides & 11 \\
\hline PCBs & 12 \\
\hline Volatile halogenated alkanes & 13 \\
\hline Volatile halogenated alkenes & 14 \\
\hline Volatile aromatic hydrocarbons & 15 \\
\hline Low molecular weight aromatics & $15 \mathrm{a}$ \\
\hline Volatile chlorinated aromatic hydrocarbons & 16 \\
\hline Volatile unsaturated carbonyl compounds & 17 \\
\hline Aldehyde & $17 \mathrm{a}$ \\
\hline Volatile ethers & 18 \\
\hline Cyclic ether & $18 \mathrm{a}$ \\
\hline Volatile ketones & 19 \\
\hline Miscellaneous volatile compounds & 20 \\
\hline Inductively coupled plasma (ICP) metals (added group) & 21 \\
\hline Non ICP cations (added group) & 22 \\
\hline Anions (added group) & 23 \\
\hline Halides & $23 a$ \\
\hline Single analyte methods (added group) & 24 \\
\hline Miscellaneous semivolatile compounds (added group) & 25 \\
\hline Alkly phosphates & $25 \mathrm{a}$ \\
\hline Long chain alkanes & $25 \mathrm{~b}$ \\
\hline Radioactive & 26 \\
\hline
\end{tabular}

* Treatability Groups based on "A Project Manager's Guide to Requesting and Evaluating Chemical Analysis", August 1991, EPA Contract No. 68D80085. 
Table 4-3. Organic Constituent Modified Treatability Envelope.

\begin{tabular}{|c|c|c|c|c|c|c|c|}
\hline $\begin{array}{l}\text { Major } \\
\text { group }\end{array}$ & Chemical Name & CAS & $\begin{array}{l}\mathrm{HBL} \\
\mathrm{mg} / \mathrm{L}\end{array}$ & $\begin{array}{c}\text { HBL Value } \\
\text { used for TBD } \\
(\mathrm{mg} / \mathrm{L})\end{array}$ & $\mathrm{EE} / \mathrm{O}^{(2)}$ & $\begin{array}{l}\text { Limiting } \\
\text { factor }^{(3)}\end{array}$ & $\begin{array}{l}\text { Maximum Influent } \\
\text { Concentration } \\
\text { Envelope to Meet } \\
10^{*} \mathrm{HBL}(\mathrm{mg} / \mathrm{L})\end{array}$ \\
\hline 1 & 2,4-Dimethylphenol & $105-67-9$ & 0.7 & & 10 & $\mathrm{~S}$ & $5.90 \mathrm{E}+02$ \\
\hline 1 & Coal tar creosote & $8007-45-2$ & TBD & 1 & 10 & $\mathrm{~s}$ & $1.00 \mathrm{E}+02$ \\
\hline 1 & Creosote & $8001-58-9$ & TBD & 1 & 10 & $S$ & $1.00 \mathrm{E}+02$ \\
\hline 1 & Cresol [Cresylic acid] (DL) ${ }^{5}$ & 1319-77-3 & 2 & & 10 & $\mathbf{s}$ & $3.10 E+04$ \\
\hline 1 & m-Cresol [3-Methylphenol] & $108-39-4$ & TBD & 1 & 10 & S & $2.50 \mathrm{E}+04$ \\
\hline 1 & o-Cresol [2-Methylphenol] & $95-48-7$ & TBD & 1 & 10 & 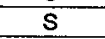 & $2.50 \mathrm{E}+04$ \\
\hline 1 & p-Cresol [4-Methylphenol] & $106-44-5$ & TBD & 1 & 10 & $s$ & $2.50 E+04$ \\
\hline 1 & Phenol & $108-95-2$ & 20 & & 4 & $\mathrm{~s}$ & $9.30 \mathrm{E}+04$ \\
\hline 2 & 2,3,4,6-Tetrachlorophenol & $58-90-2$ & 1 & & 10 & $s$ & $1.00 E+03$ \\
\hline 2 & $\begin{array}{l}\text { 2,3,4,6-tetrachlorophenol, potassium salt } \\
{[2,3,4,6 \text {-tetrachlorophenol salt] }}\end{array}$ & $53535-27-6$ & TBD & 1 & 10 & $\mathrm{~s}$ & $1.00 \mathrm{E}+03$ \\
\hline 2 & $\begin{array}{l}\text { 2,3,4,6-tetrachlorophenol, sodium salt }[2,3,4,6- \\
\text { tetrachlorophenol, salt }]\end{array}$ & 25567-55-9 & TBD & 1 & 10 & $s$ & $1.00 \mathrm{E}+03$ \\
\hline 2 & 2,4,5-Trichlorophenol & $95-95-4$ & 4 & & 10 & $s$ & $1.19 E+03$ \\
\hline 2 & 2,4,6-Tribromophenol & 118-79-6 & TBD & 1 & 10 & $s$ & $7.00 \mathrm{E}+01$ \\
\hline 2 & 2,4,6-Trichlorophenol & $88-06-2$ & 0.008 & & 10 & $s$ & $8.00 E+02$ \\
\hline 2 & 2,4-Dichlorophenol & $120-83-2$ & 0.1 & & 10 & $L$ & $2.00 \mathrm{E}+03$ \\
\hline 2 & 2,6-Dichlorophenol & $87-65-0$ & TBD & 1 & 10 & $L$ & $2.00 \mathrm{E}+03$ \\
\hline 2 & m-Chloro-3-methylphenol & $59-50-7$ & TBD & 1 & 10 & $\bar{L}$ & $2.00 E+03$ \\
\hline 2 & o-Chlorophenol & $95-57-8$ & 0.2 & & 10 & $\underline{L}$ & $2.00 \mathrm{E}+03$ \\
\hline 2 & Pentachlorophenol & $87-86-5$ & 0.001 & & 4 & s. & $1.40 \mathrm{E}+01$ \\
\hline 2 & $\begin{array}{l}\text { Potassium pentachlorophenate } \\
\text { [Pentachlorophenol salt] }\end{array}$ & $7778-73-6$ & 0.001 & $\cdot$ & 4 & $L$ & $1.00 \mathrm{E}+05$ \\
\hline 2 & $\begin{array}{l}\text { Sodium pentachlorophenate [Pentachlorophenol } \\
\text { salt] }\end{array}$ & $131-52-2$ & 0.001 & & 4 & $\mathrm{~L}$ & $1.00 \mathrm{E}+05$ \\
\hline 3 & Acenaphthene & $83-32-9$ & 2 & & 10 & $S$ & $3.42 E+00$ \\
\hline 3. & Acenaphthylene & 208-96-8 & TBD & 1 & 10 & $\mathrm{~s}$ & $3.93 \mathrm{E}+00$ \\
\hline 3 & Anthracene & $120-12-7$ & 10 & . & 10 & $\mathrm{~s}$ & $1.29 \mathrm{E}+00$ \\
\hline 3 & Benzene (DL) ${ }^{5}$ & $71-43-2$ & 0.005 & & 3 & $s$ & $1.75 \mathrm{E}+03$ \\
\hline 3 & Benzenearsonic acid (Arsenic) & 98-05-5 & TBD & 1 & 10 & $s$ & $2.50 \mathrm{E}+04$ \\
\hline 3 & Fluorene & $86-73-7$ & 1 & & 10 & $\mathrm{~s}$ & $1.69 \mathrm{E}+00$ \\
\hline
\end{tabular}


蒙

产

E

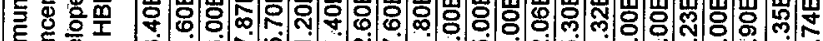

齐

이융ํํํํำ

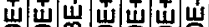

ㅇㅇㅇ융ㅇㅇㅇㅇㅇㅇㅛ 는 운

-

을

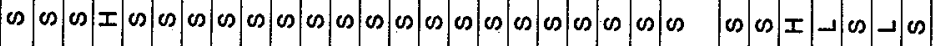

(9)

Q

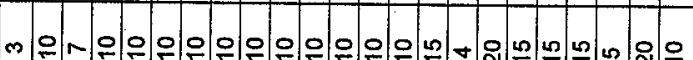

过

W

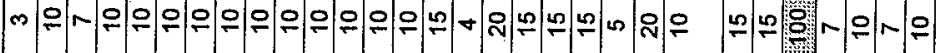

$\Phi \stackrel{0}{\mathrm{~m}}$

का

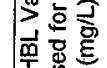

工

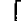

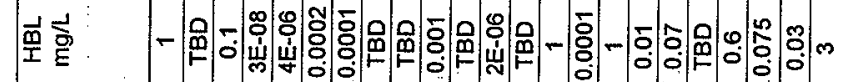

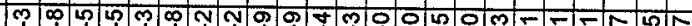

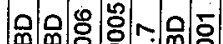

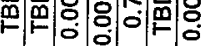

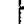

$\frac{0}{0}$

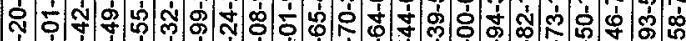

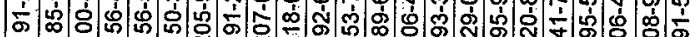

\%नण

ผ

œ

\% ヘิ

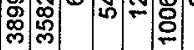

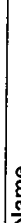

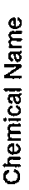

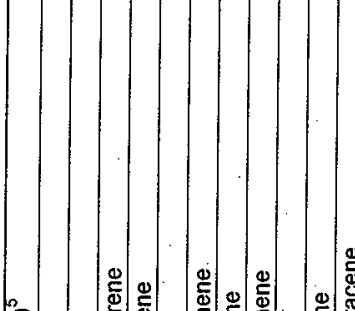

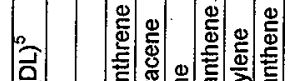

$\stackrel{\$}{\frac{D}{8}}$

竞

동

خัخ

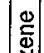

(n) $\div$

(1)

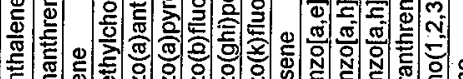

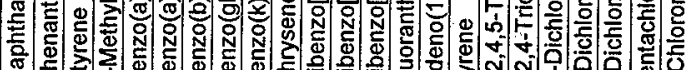

Z

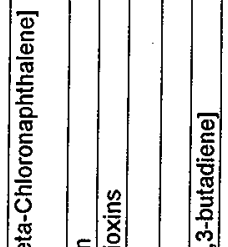

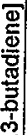

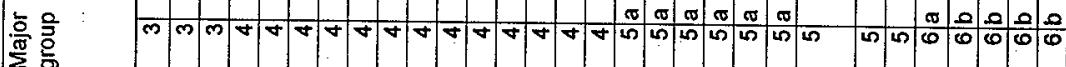


Table 4-3. Organic Constituent Modified Treatability Envelope.

\begin{tabular}{|c|c|c|c|c|c|c|c|}
\hline $\begin{array}{l}\text { Major } \\
\text { group }\end{array}$ & Chemical Name & CAS & $\begin{array}{l}\mathrm{HBL} \\
\mathrm{mg} / \mathrm{L}\end{array}$ & $\begin{array}{l}\text { HBL Value } \\
\text { used for TBD } \\
\text { (mg/L) }^{(1)}\end{array}$ & $\mathrm{EE} / \mathrm{O}^{(2)}$ & $\begin{array}{l}\text { Limiting } \\
\text { factor }\end{array}$ & $\begin{array}{l}\text { Maximum Influent } \\
\text { Concentration } \\
\text { Envelope to Meet } \\
10^{*} \mathrm{HBL}(\mathrm{mg} / \mathrm{L})\end{array}$ \\
\hline 6$] \mathrm{b}$ & Hexachloropropene & 1888-71-7 & 0.01 & 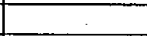 & 10 & $\mathrm{~s}$ & $4.00 \mathrm{E}-03$ \\
\hline $7 a$ & bis(2-Chloroethoxy) methane & $111-91-1$ & TBD & 1 & 15 & $\mathrm{~L}$ & $2.00 E+03$ \\
\hline $7 a$ & Bis(2-Chloroethyl) ether & 111-44-4 & 0.00008 & & 5 & $\mathbf{L}$ & $2.00 \mathrm{E}+03$ \\
\hline \begin{tabular}{l|l}
7 & $a$ \\
\end{tabular} & Bis(2-Chloroisopropyl) ether & $39638-32-9$ & 0.001 & & 15 & $\mathrm{H}$ & $1.90 \mathrm{E}+02$ \\
\hline $7 a$ & Dichloroisopropyl ether & $108-60-1$ & 0.001 & & 15 & $\mathrm{H}$ & $1.90 \mathrm{E}+02$ \\
\hline 7 b & 4-Bromophenylphenyl ether & $101-55-3$ & TBD & 1 & 10 & $\mathrm{~s}$ & $1.00 \mathrm{E}+00$ \\
\hline 7 b & 4-Chlorophenyl phenyl ether & $7005-72-3$ & TBD & 1 & 4 & $S$ & $3.30 \mathrm{E}+00$ \\
\hline 8 & Bis(2-Ethylhexyl) phthalate & 117-81-7 & 0.006 & & 5 & $\mathrm{~s}$ & $4.00 \mathrm{E}-01$ \\
\hline 8 & Butylbenzylphthalate & $85-68-7$ & 0.1 & & 15 & $\mathrm{~s}$ & $2.90 \mathrm{E}+00$ \\
\hline 8 & Diethylphthalate & $84-66-2$ & 30 & & 15 & $\mathrm{~S}$ & $8.96 E+02$ \\
\hline 8 & Dimethyl phthalate & 131-11-3 & 400 & & 15 & $\mathrm{~s}$ & $4.30 E+03$ \\
\hline 8 & Di-n-butylphthalate & 84-74-2 & 4 & & 15 & $s$ & $1.30 \mathrm{E}+01$ \\
\hline 8 & Di-n-octylphthalate (DL) & 117-84-0 & 0.7 & & 15 & s & $3.00 E+00$ \\
\hline $9 a$ & 1-Butanol $(\mathrm{DL})^{5}$ & $71-36-3$ & 4 & & 10 & $\mathrm{~s}$ & $9.10 \mathrm{E}+04$ \\
\hline $9 a$ & Benzyl alcohol (DL) $)^{5}$ & $100-51-6$ & TBD & 1 & 10 & $S$ & $4.00 \mathrm{E}+04$ \\
\hline $9 a$ & Isobutyl alcohol & $78-83-1$ & 10 & & 20 & $\mathrm{~S}$ & $7.60 \mathrm{E}+04$ \\
\hline 9. & Methanol & $67-56-1$ & 20 & & 15 & $\mathrm{~L}$ & $5.00 E+05$ \\
\hline 9 & 1,4-Dioxane [1,4-Diethyleneoxide] & $123-91-1$ & 0.008 & & 5 & $\mathrm{~L}$ & $2.00 \mathrm{E}+03$ \\
\hline 9 & $2,4,5-T$ & $93-76-5$ & 0.4 & & 20 & $\mathrm{~S}$ & $2.40 E+02$ \\
\hline 9 & 2,4,5-TP [Silvex] & $93-72-1$ & 0.05 & & 20 & $S$ & $1.40 \mathrm{E}+02$ \\
\hline 9 & 2,4-D [2,4-Dichlorophenoxyacetic acid] & $94-75-7$ & 0.07 & & 20 & $s$ & $8.90 \mathrm{E}+02$ \\
\hline 9 & Carbofuran phenol & $1563-38-8$ & TBD & 1 & 30 & $s$ & $1.00 \mathrm{E}+00$ \\
\hline 9 & Ethyl Acetate & $141-78-6$ & 30 & & 15 & L & $1.00 \mathrm{E}+05$ \\
\hline 9 & Kepone & $143-50-0$ & $2 \mathrm{E}-06$ & & 15 & $\mathrm{H}$ & 3.80 E-01 \\
\hline 9 & Lead subacetate (Lead) & $1335-32-6$ & TBD & 1 & 15 & $\mathrm{H}$ & $1.90 \mathrm{E}+04$ \\
\hline 9 & Methyl methacrylate & $80-62-6$ & 3 & & 20 & $s$ & $2.00 E+01$ \\
\hline 9 & Methyl methanesulfonate & $66-27-3$ & TBD & 1 & 20 & $\mathrm{H}$ & $1.62 \mathrm{E}+03$ \\
\hline 9 & Phenyl mercuric acetate & $62-38-4$ & TBD & 1 & 15 & $\mathrm{~s}$ & $4.37 E+03$ \\
\hline 9 & Phthalic anhydride & $85-44-9$ & TBD & 1 & 15 & $\mathrm{~L}$ & $2.00 \mathrm{E}+03$ \\
\hline 9 & Safrole & $94-59-7$ & 0.0005 & & 20 & $\mathrm{H}$ & $8.10 E+00$ \\
\hline 9 & TCDD & 1746-01-6 & TBD & 1 & 15 & 5 & 2.00E-05 \\
\hline
\end{tabular}




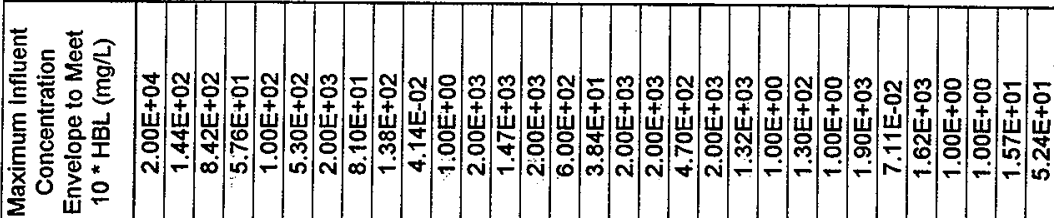

오옹

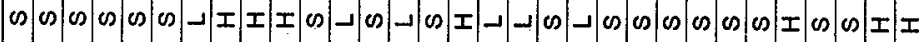

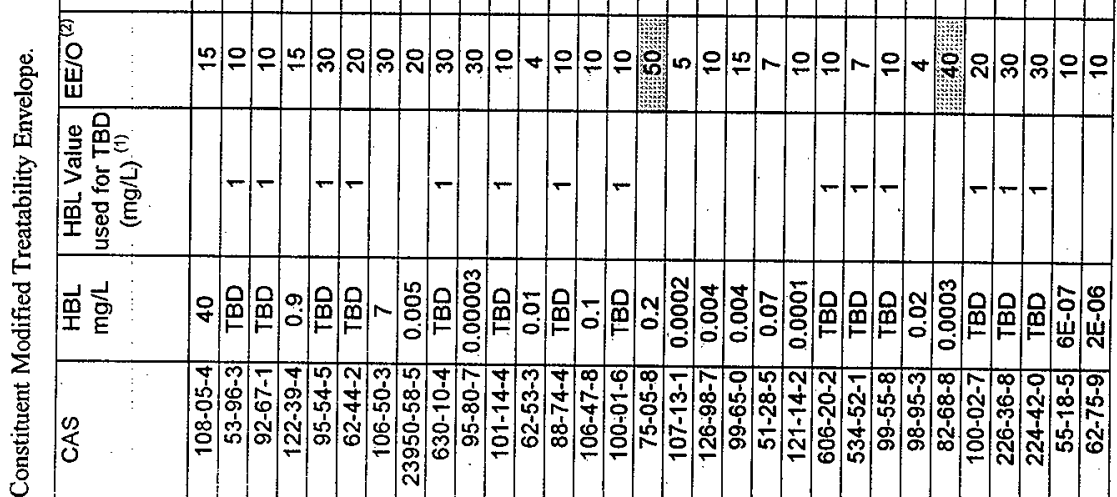

莺

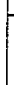

3
$\dot{y}$
0
0

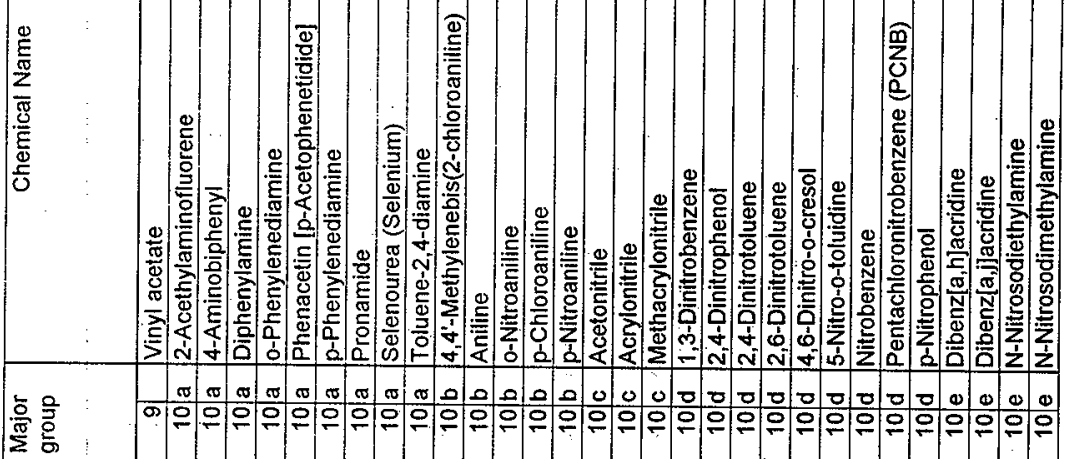


Table 4-3. Organic Constituent Modified Treatability Envelope.

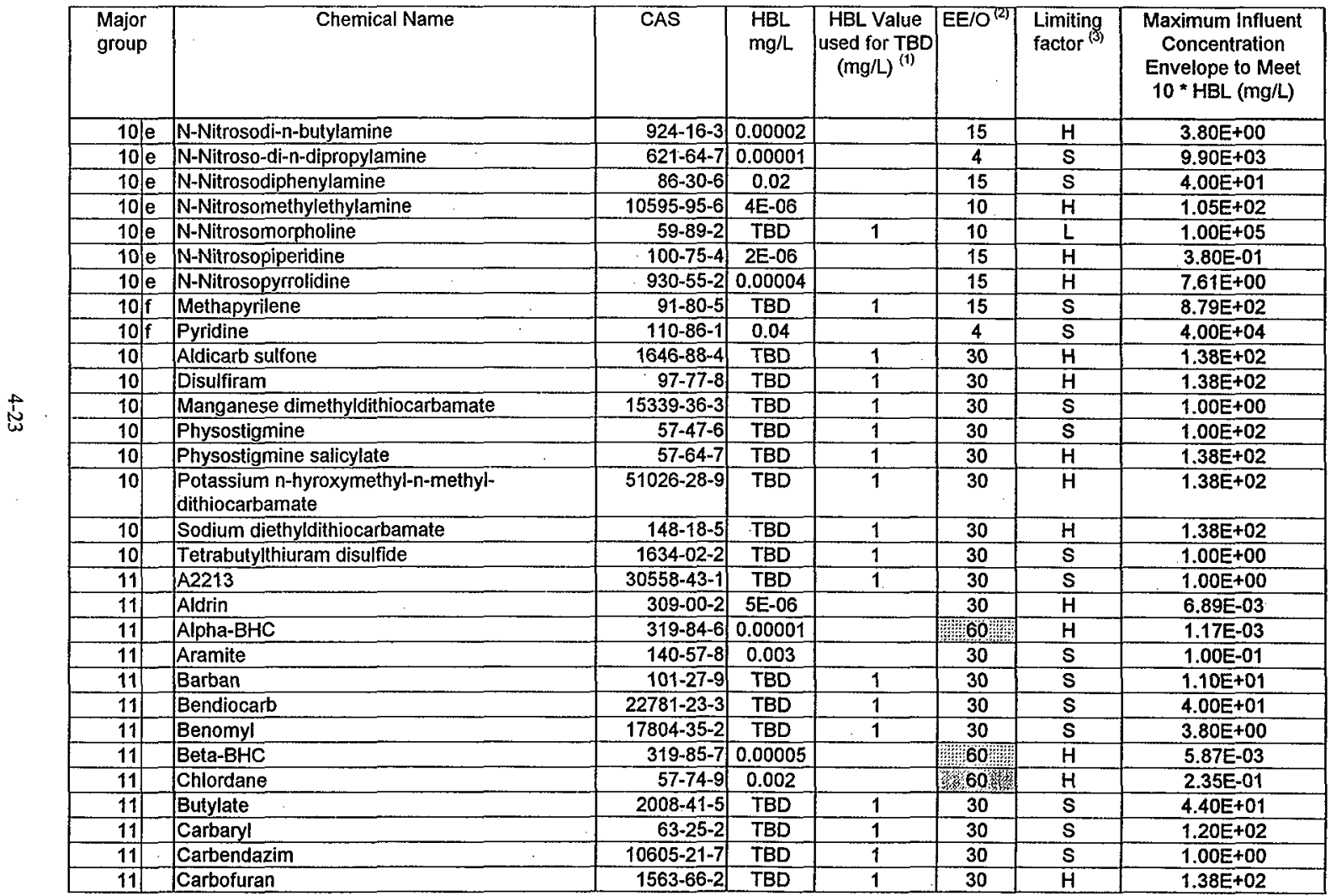


Table 4-3. Organic Constituent Modified Treatability Envelope.

\begin{tabular}{|c|c|c|c|c|c|c|c|}
\hline $\begin{array}{l}\text { Major } \\
\text { group }\end{array}$ & Chemical Name & . & $\begin{array}{l}\mathrm{HBL} \\
\mathrm{mg} / \mathrm{L}\end{array}$ & $\begin{array}{c}\text { HBL Value } \\
\text { used for TBD } \\
\text { (mg/L) }^{(1)}\end{array}$ & $\begin{array}{c}\mathrm{EE} / \mathrm{O}^{(2)} \\
\cdots\end{array}$ & $\begin{array}{l}\text { Limiting } \\
\text { factor }_{(3)}\end{array}$ & $\begin{array}{l}\text { Maximum Influent } \\
\text { Concentration } \\
\text { Envelope to Meet } \\
10^{*} \mathrm{HBL}(\mathrm{mg} / \mathrm{L})\end{array}$ \\
\hline 11) & Copper dimethyldithiocarbamate & $137-29-1$ & TBD & 1 & 30 & $\mathrm{~S}$ & $1.00 \mathrm{E}+00$ \\
\hline 11) & DDD $\left(p, p^{\prime \prime}-D D D\right)$ & $72-54-8$ & 0.0004 & & 30 & $S$ & 1.00E-01 \\
\hline 11 & DDE $\left(p, p^{\prime}-D D E\right)$ & $72-55-9$ & 0.0003 & & 30 & $\mathrm{~s}$ & $4.00 E-02$ \\
\hline 11 & DDT $\left(p, p^{\prime}-D D T\right)$ & $50-29-3$ & 0.0003 & & 30 & 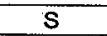 & $5.00 \mathrm{E}-03$ \\
\hline 11 & Delta-BHC & $319-86-8$ & TBD & 1 & 60 & S & $1.00 E+01$ \\
\hline 11 & Dieldrin & $60-57-1$ & $5 \mathrm{E}-06$ & & 30 & $\mathrm{H}$ & $6.89 \mathrm{E}-03$ \\
\hline 11 & Dinoseb & $88-85-7$ & 0.007 & & 30 & $\mathrm{H}$ & $9.65 \mathrm{E}+00$ \\
\hline 11 & Disolfoton & 298-04-4 & 0.001 & & 20 & $\mathrm{H}$ & $1.62 E+01$ \\
\hline 11 & Endosulfan I & 959-98-8 & TBD & 1 & 20 & $\mathbf{S}$ & $1.00 E+00$ \\
\hline 11 & Endosulfan II & $33213-65-9$ & TBD & 1 & 20 & $S$ & $1.00 \mathrm{E}+00$ \\
\hline 11 & Endosulfan sulfate & $1031-07-8$ & TBD & 1 & 20 & $S$ & 1:17E-01 \\
\hline 11 & Endrin & $72-20-8$ & 0.002 & & 20 & $\mathrm{~S}$ & $2.50 \mathrm{E}-01$ \\
\hline 11. & Endrin aldehyde & $7421-93-4$ & TBD & 1 & 20 & $S$ & $2.50 \mathrm{E}-01$ \\
\hline 11 & EPTC & $759-94-4$ & TBD & 1 & 30 & $\mathrm{H}$ & $1.38 \mathrm{E}+02$ \\
\hline 11 & Ethyl Ziram & 14324-55-1 & TBD & 1 & 30 & $s$ & $1.00 E+00$ \\
\hline 11 & Famphur & $52-85-7$ & 0.001 & & 20 & $\mathrm{H}$ & $1.62 \mathrm{E}+01$ \\
\hline 11 & Ferbam & 14484-64-1 & TBD & 1 & 30 & $s$ & $1.20 \mathrm{E}+02$ \\
\hline 11 & Heptachlor & $76-44-8$ & 0.0004 & & 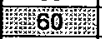 & $\mathrm{H}$ & $4.70 \mathrm{E}-02$ \\
\hline 11 & Heptachlor epoxide & $1024-57-3$ & 0.0002 & & 20 & $s$ & $3.50 \mathrm{E}-01$ \\
\hline 11 & Isodrin & $465-73-6$ & TBD & 1 & 20 & $S$ & $1.00 E+00$ \\
\hline 11 & Lindane [gamma-BHC] & $58-89-9$ & 0.0002 & & 40 & $\mathrm{H}$ & $8.05 E-02$ \\
\hline 11 & Metam Sodium & $137-42-8$ & TBD & 1 & 30 & $\mathrm{H}$ & $1.38 \mathrm{E}+02$ \\
\hline 11 & Methiocarb & 2032-65-7 & TBD & 1 & 30 & $\mathrm{~s}$ & $1.00 \mathrm{E}+00$ \\
\hline 11 & Methoxychlor & $72-43-5$ & 0.04 & & 20 & $\mathrm{~S}$ & 4.00E-02 \\
\hline 11 & Methyl parathion & 298-00-0 & 0.009 & & 20 & $\mathbf{S}$ & $6.00 \mathrm{E}+01$ \\
\hline 11 & Mexacarbate & $315-18-4$ & TBD & 1 & 30 & $s$ & $1.00 \mathrm{E}+02$ \\
\hline 11 & Molinate & 2212-67-1 & TBD & 1. & 30 & $\mathrm{H}$ & $1.38 \mathrm{E}+02$ \\
\hline 11 & $0, p^{\prime}-D D D$ & $53-19-0$ & TBD & 1 & 30 & $\mathbf{S}$ & $1.00 E+00$ \\
\hline 11 & $0, p^{\prime}-D D E$ & $3424-82-6$ & TBD & 1 & 30 & $\mathbf{s}$ & $1.00 E+00$ \\
\hline 11 & o.p'-DDT & $789-02-6$ & TBD & 1 & 30 & $\mathrm{~s}$ & $1.00 E+00$ \\
\hline 11 & Oxamyl & $23135-22-0$ & TBD & 1 & 30 & $\bar{H}$ & $1.38 E+02$ \\
\hline
\end{tabular}




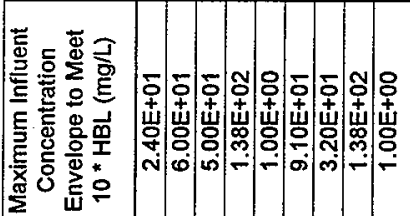

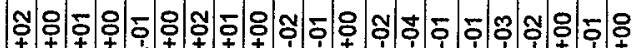

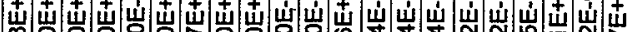

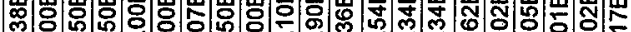

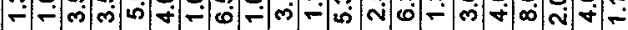

을

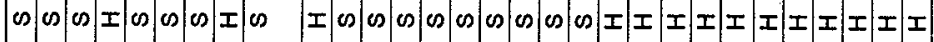

के

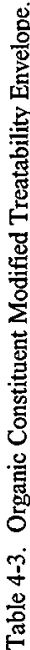

过

ii.

$-1$

H

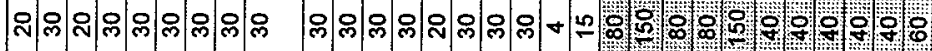

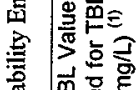

$\frac{\infty}{5}$

$$
\text { 重兽 }
$$$$
-H--H-H-C-
$$

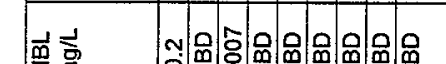

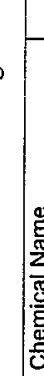

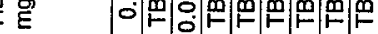

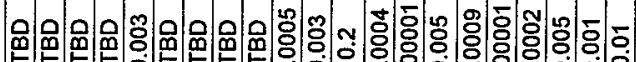

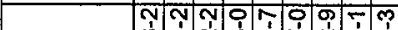

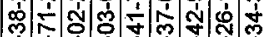

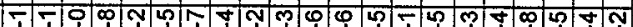

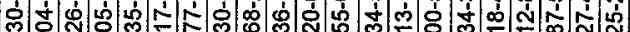

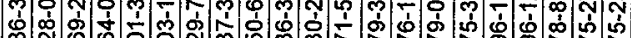

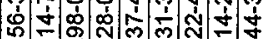

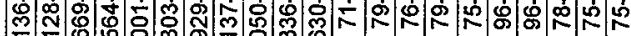
F $\because \div=$

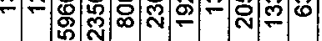


Table 4-3. Organic Constituent Modified Treatability Envelope.

\begin{tabular}{|c|c|c|c|c|c|c|c|}
\hline $\begin{array}{l}\text { Major } \\
\text { group } \\
.\end{array}$ & \begin{tabular}{cccc} 
& & \multicolumn{3}{c}{ Chemical Name } \\
& & & \\
& & &
\end{tabular} & CAS & $\begin{array}{l}\mathrm{HBL} \\
\mathrm{mg} / \mathrm{L}\end{array}$ & $\begin{array}{c}\text { HBL Value } \\
\text { used for TBD } \\
\text { (mg/L) }^{(1)}\end{array}$ & $E=/ O^{(2)}$ & $\begin{array}{l}\text { Limiting } \\
\text { factor }{ }^{(3)} \\
\end{array}$ & $\begin{array}{l}\text { Maximum Influent } \\
\text { Concentration } \\
\text { Envelope to Meet } \\
10^{*} \mathrm{HBL}(\mathrm{mg} / \mathrm{L})\end{array}$ \\
\hline 13 & Bromomethane [Methyl bromide] & $74-83-9$ & 0.05 & & 40 & $\mathrm{H}$ & $2.01 E+01$ \\
\hline 13 & Carbon tetrachloride $(\mathrm{DL})^{5}$ & $56-23-5$ & 0.005 & & $200 \%$ & $\mathrm{H}$ & $1.05 E-01$ \\
\hline 13. & Chloroethane & $75-00-3$ & TBD & 1 & 40 & $\mathrm{H}$ & $4.02 E+01$ \\
\hline 13 & Chloroform (DL) & $67-66-3$ & 0.01 & & 100 & $\mathrm{H}$ & 4.38E-01 \\
\hline 13 & Chloromethane [Methyl chloride] & $74-87-3$ & 0.007 & & 30 & $\mathrm{H}$ & $9.65 \mathrm{E}+00$ \\
\hline 13 & Dibromochloromethane & $124-48-1$ & 0.001 & & 40 & $\mathrm{H}$ & $4.02 E-01$ \\
\hline 13. & Dibromomethane [Methylene bromide] & $74-95-3$ & 0.4 & & 30 & $\mathrm{H}$ & $5.52 E+02$ \\
\hline 13 & Dichlorodifluoromethane & $75-71-8$ & 7 & & 80 & $S$ & $2.80 \mathrm{E}+02$ \\
\hline 13 & Ethylene dibromide [1,2-Dibromoethane] & $106-93-4$ & 0.00005 & & $40: 4$ & $\mathrm{H}$ & 2.01E-02 \\
\hline 13 & Ethylene dichloride [1,2-Dichloroethane] (DL) ${ }^{5}$ & $1.07-06-2$ & 0.005 & & 40 & $\mathbf{H}$ & $2: 01 E+00$ \\
\hline 13 & lodomethane [Methyl iodide] & $74-88-4$ & TBD & 1 & 30 & $\mathrm{H}$ & $1.38 \mathrm{E}+02$ \\
\hline 13. & Methylene chloride [Dichloromethane] & $75-09-2$ & 0.005 & & 60 & $\mathrm{H}$ & 5.87E-01 \\
\hline 13 & Trichlorofluoromethane & $75-69-4$ & 10 & & 80.4 & $\mathrm{H}$ & $6.34 E+02$ \\
\hline 14 & 1,1-Dichloroethylene (DL) ${ }^{5}$ & $75-35-4$ & 0.007 & & 4 & $L$ & $2.00 \mathrm{E}+03$ \\
\hline 14 & Allyl chloride [3-Chloropropene] & 107-05-1 & 0.004 & & 5 & $\mathbf{S}$ & $1.00 \mathrm{E}+02$ \\
\hline 14. & cis- 1,2 -Dicloroethylene & $156-59-2$ & 0.07 & & 5 & $\mathrm{~L}$ & $2.00 \mathrm{E}+03$ \\
\hline 14 & Tetrachloroethene [Tetrachloroethylene] $(\mathrm{DL})^{5}$ & $127-18-4$ & 0.005 & & 4 & $s$ & $1.50 E+02$ \\
\hline 14 & trans-1,2-Dichloroethylene & $156-60-5$ & 0.1 & & 5 & $\underline{L}$ & $2.00 E+03$ \\
\hline 14 & trans-1,3-Dichloropropene & $10061-02-6$ & TBD & 1 & 5 & $\bar{L}$ & $2.00 \mathrm{E}+03$ \\
\hline 14 & Trichloroethylene (DL) $)^{5}$ & $79-01-6$ & 0.005 & & 4 & $\mathbf{s}$ & $1.10 \mathrm{E}+03$ \\
\hline 14 & Vinyl chloride (DL) ${ }^{5}$ & $75-01-4$ & 0.002 & & 4 & $L$ & $2.00 E+03$ \\
\hline \begin{tabular}{l|l}
15 & $\mathbf{a}$ \\
\end{tabular} & Ethylbenzene & $100-41-4$ & 0.7 & & 7 & $\mathbf{S}$ & $1.52 \mathrm{E}+02$ \\
\hline $15 \mid a$ & Toluene (DL) ${ }^{5}$ & 108-88-3 & 1 & & 2 & $\mathbf{s}$ & $5.35 E+02$ \\
\hline \begin{tabular}{l|l|l}
15 & $a$ \\
\end{tabular} & Xylenes (total) & $1330-20-7$ & 10 & & 7 & $s$ & $1.98 \mathrm{E}+02$ \\
\hline 16 & Chlorobenzene (DL) ${ }^{5}$ & 108-90-7 & 0.1 & & 5 & s & $4.66 \mathrm{E}+02$ \\
\hline 16 & Dichlorophenylarsine (Arsenic) & $696-28-6$ & TBD & & 4 & $\mathrm{~s}$ & $1.00 \mathrm{E}+00$ \\
\hline \begin{tabular}{l|l}
17 & $\mathbf{a}$ \\
\end{tabular} & Acrolein & $107-02-8$ & 0.7 & & 4 & $\mathrm{~L}$ & $2.00 \mathrm{E}+03$ \\
\hline \begin{tabular}{l|l|}
18 & $a$ \\
\end{tabular} & Isosafrole & $120-58-1$ & TBD & 1 & 15 & S & $1.00 E+00$ \\
\hline 18 & Ethyl ether & $60-29-7$ & 7 & & 20 & $\underline{L}$ & $2.00 E+03$ \\
\hline 18 & Ethyl methacrylate & $97-63-2$ & 3 & & 20 & $S$ & $7.00 E+02$ \\
\hline
\end{tabular}


Table 4-3. Organic Constituent Modified Treatability Envelope.

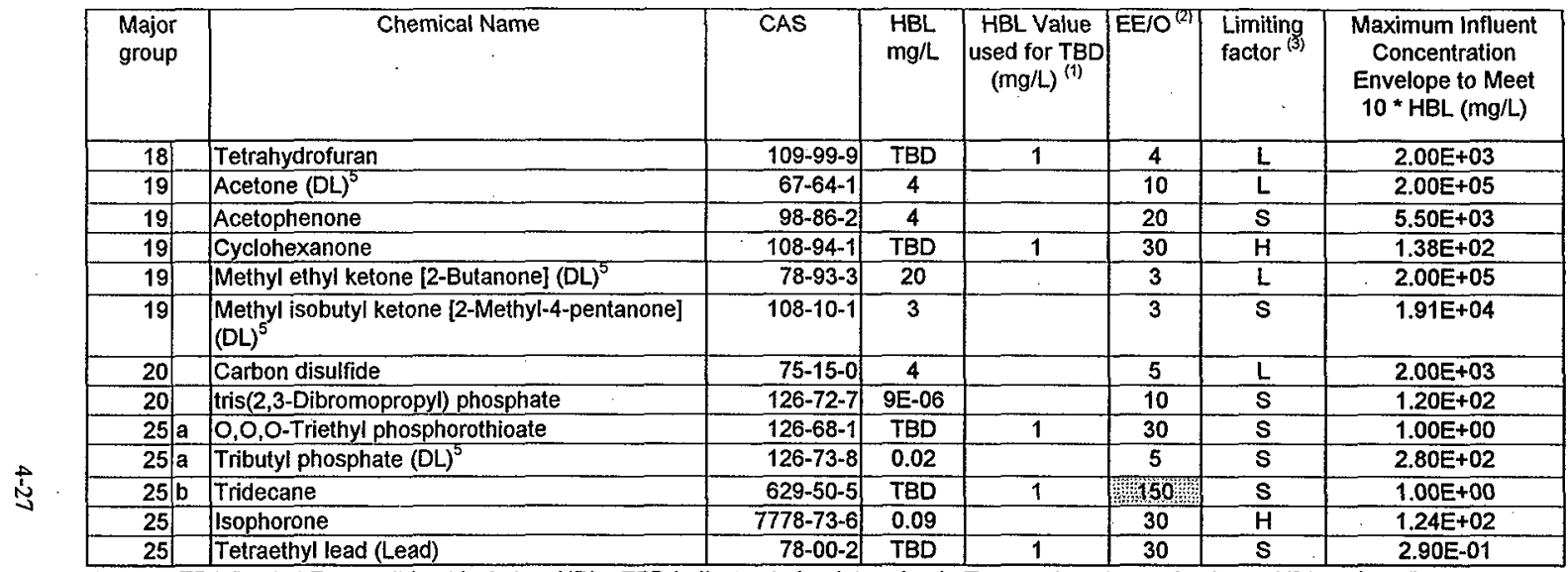

(1) The EPA Docket Report did not include a HBL. TBD indicates to be determined. To complete the evaluation, a HBL of $1 \mathrm{mg} / \mathrm{L}$ was assumed. When a HBL is established for an organic constituent not included in the referenced Docket Report, the maximum influent concentration envelope at 10 times the $\mathrm{HBL}$ is determined by multiplying the newly determined $\mathrm{HBL}$ by the maximum influent concentration envelope from this table.

(2) $\mathrm{EE} / \mathrm{O}$ is defined in Section 4.1. Shading indicates hard to treat organic compounds.

(3) The maximum influent concentration envelope depends on one of three factors: $H$ indicates the envelope is based on 10 times $H B L$, $L$ indicates the envelope is limited by the LERF liner compatibility, $S$ indicates the envelope is limited by the organic compound solubility. Refer to Appendix $B$ for solubility data and LERF liner compatibility data.

(4) The ETF influent waste concentration where following upon once-through treatment, the treated waste concentration is less than 10 times the HBL.

(5) DL indicates a delisting limit established in the final delisting (40 CFR 261 Appendix IX, Table 2).

CAS $=$ Chemical Abstract Service.

$E E / O=$ Electrical Energy per Order.

$\mathrm{HBL}=$ health-based level.

$\mathrm{mg} / \mathrm{L}=$ milligrams per liter .

$\mathrm{TBD}=$ to be determined. 
Table 4-4. Standards Treated Waste Must Meet.

\begin{tabular}{|c|c|c|c|c|}
\hline \multirow[t]{2}{*}{ Constituent } & \multirow[t]{2}{*}{$\begin{array}{l}\text { Delisting limit } \\
\text { (micrograms } \\
\text { per liter). }\end{array}$} & \multicolumn{3}{|c|}{$\begin{array}{l}\text { Washington State Waste Discharge } \\
\text { Permit limits (micrograms per liter) }\end{array}$} \\
\hline & & \begin{tabular}{|c|}
$\begin{array}{c}\text { Enforcement limit } \\
\text { monthly average }\end{array}$ \\
\end{tabular} & $\begin{array}{c}\text { Enforcement limit } \\
\text { daily maximum }\end{array}$ & $\begin{array}{c}\text { Early waming } \\
\text { value }\end{array}$ \\
\hline 1,1,1-Trichloroethane & 2,000 & & & \\
\hline 1,1,2-Trichloroethane & 50 & & & 5 \\
\hline 1,1-Dichloroethene & 70 & & & \\
\hline 1,2-Dichloroethane & 50 & & & \\
\hline 1,4-Dichlorobenzene & 750 & & & \\
\hline 1-Butanol & 40,000 & & & \\
\hline Methyl ethyl ketone & 200,000 & & & \\
\hline Methyl isobutyl ketone & 30,000 & & & \\
\hline Acetone & 40,000 & & & \\
\hline Acetophenone & & & & 10 \\
\hline Ammonia (as N) & & & & 830 \\
\hline Ammonium & 10,000 & & & \\
\hline Antimony & 60 & & & \\
\hline Arsenic & 500 & 15 & 30 & \\
\hline Barium & 20,000 & & & \\
\hline Benzene & 50 & r. & & 5 \\
\hline Benzyl alcohol & 100,000 & & & \\
\hline Beryllium & 40 & & & 40 \\
\hline Cadmium & 50 & & & 7.5 \\
\hline Carbon tetrachloride & 50 & 5 & 10 & \\
\hline Chloride & & & & 250,000 \\
\hline Chlorobenzene & 1,000 & & & \\
\hline Chloroform & 100 & & & 5 \\
\hline Chromium & 1,000 & 20 & & \\
\hline Copper & & & & 70 \\
\hline Cyanide & 2,000 & & & \\
\hline Di-n-octyl phthalate & 7,000 & & & \\
\hline Fluoride & 40,000 & & & \\
\hline Hexachloroethane & 60 & & & \\
\hline Lead & 150 & & & 38 \\
\hline Mercury & 20 & & & 2 \\
\hline Naphthalene & 10,000 & & & \\
\hline Nickel & 1,000 & & & \\
\hline Nitrate (as N) & & 3,800 & & \\
\hline Nitrite (as N) & & & & 100 \\
\hline Nitrogen total (TKN) & & & & 600 \\
\hline $\mathrm{N}$-Nitrosodimethylamine & & 20 & & \\
\hline Selenium & 500 & & & \\
\hline Silver & 2,000 & & & \\
\hline Sulfate & & 10,000 & & \\
\hline Tetrachloroethylene & 50 & 5 & 10 & \\
\hline Tetrahydrofuran & & & & 100 \\
\hline
\end{tabular}


Table 4-4. Standards Treated Waste Must Meet.

\begin{tabular}{|l|c|c|c|c|}
\hline \multicolumn{1}{|c|}{ Constituent } & $\begin{array}{c}\text { Delisting limit } \\
\text { (micrograms } \\
\text { per liter) }\end{array}$ & \multicolumn{3}{c|}{$\begin{array}{c}\text { Washington State Waste Discharge } \\
\text { Permit limits (micrograms per liter) }\end{array}$} \\
\cline { 4 - 5 } & & $\begin{array}{c}\text { Enforcement limit } \\
\text { monthly average }\end{array}$ & $\begin{array}{c}\text { Enforcement limit } \\
\text { daily maximum }\end{array}$ & $\begin{array}{c}\text { Early warning } \\
\text { value }\end{array}$ \\
\hline Toluene & 10,000 & & & \\
\hline Total cresol & 20,000 & & & 380,000 \\
\hline Total dissolved solids & & & & 1,100 \\
\hline Total organic carbon & & & & 4,000 \\
\hline Total suspended solids & & & & \\
\hline Tributyl phosphate & 200 & & & \\
\hline Trichloroethylene & 50 & & & \\
\hline Vanadium & 2,000 & & & \\
\hline Vinyl chloride & 20 & & & \\
\hline Zinc & 100,000 & & & \\
\hline
\end{tabular}


This page intentionally left blank. 


\subsection{SAMPLING AND ANALYSIS}

The ETF/LERF sampling and analysis, as part of the waste analysis plan, is detailed in the Hanford Facility RCRA Permit (Chapter 4). The waste analysis plan includes sections that define the ETF/LERF process for accepting waste and the sample analysis requirements for verification tank samples to confirm that the waste is treated below the regulatory limits before disposal.

The leachate to be treated at the ETF will be generated from solid waste identified in Chapter 2.0 as a typical distribution of waste to be managed at the LLBG. Using Table 2-1 as a typical distribution of what the leachate might contain and the treatability evaluation, detailed in Chapter 4.0 , strong evidence is provided that no additional delisting limits or analytical requirements need to be imposed by the waste analysis plan as a result of treating multi-source leachate. The following reasons justify this recommendation.

- There are only three organic compounds that are hard to treat and are greater than one-tenth of one percent of the typical waste volume to be disposed at the LLBG (refer to Table 2-1). These three compounds, 1,2-dichloroethane, carbon tetrachloride, and chloroform, currently have delisting limits as part of the Final Delisting (40 CFR 261 Appendix IX, Table 2). Based on the treatability analysis in Chapter 4.0, the halogenated hydrocarbon treatability groups (e.g., volatile halogenated alkanes and chlorinated aliphatic hydrocarbons) are the most difficult to treat. This is consistent with the conclusion drawn in the initial delisting petition. The sample and analysis plan currently includes these compounds.

- There are seven other organics that are hard to treat and are each less than one-tenth of one percent of the typical waste volume to be disposed at the LLBG (refer to Table 2-1). Of the seven compounds, hexachloroethane and 1,1,1-trichloroethane have delisting levels and are included in the sample and analysis plan. The other five compounds are acetonitrile, chlordane, heptachlor, methylene chloride, and trichlorofluorormethane. As shown in Table 2-1, only 17 waste containers have a waste designation including these five compounds. Methylene chloride, a common laboratory contaminate, is also included in the sample and analysis plan. Because of the very low percentage of waste volume designated due to these compounds and the fact that the LDR requirements for these organic compounds must be met before disposal at the LLBG, these compounds will not be included in the analytical requirements.

- Sulfide does not have a delisting limit and currently is identified as being associated with less than one percent of the waste identified for disposal at the LLBG. Sulfide is readily removed at the ETF, refer to Section 4.3. Cyanide is used to represent sulfide because it is associated with a much larger volume of waste and, as with sulfide, is removed easily by the ETF processes as shown in the initial delisting petition (DOE/RL-92-72). Cyanide currently has a delisting limit. Using a constituent to represent other constituents is a basic tenant of the treatability group concept.

- Cobalt, osmium, thallium, and tin are not identified as metals in the typical waste volume to be disposed at the LLBG (refer to Table 2-1). These metals are removed easily at the ETF (refer to Section 4.3). Mercury, a metal expected in waste disposed at the LLBG, has the same HBL as thallium and is used to represent thallium. The other three metals have representative metals based on similar valance states. Cobalt is represented by zinc, and osmium and tin are represented by selenium. Mercury, selenium, and zinc currently have delisting limits and are included in the sample and analysis plan. Using a constituent to represent other constituents is a basic tenant of the treatability group concept. 
1 For these reasons, no additional delisting limits are required, no additional analytical requirements are

2 needed, and no changes are required in the verification tank sampling frequency. The requirements in the 3 current waste analysis plan ensure that LERF/ETF treatment and storage of multi-source leachate is 4 monitored to ensure the waste is rendered nonhazardous and nondangerous before disposal.

5

6

7

8

9

Given the upfront delisting approach employed by this delisting modification document, sampling and analysis information required by 40 CFR $260.22(\mathrm{i})(1),(2),(3),(10)$, and (11) is not available. 


\subsection{F039 LEACHATE DELISTING CONCLUSION}

3 The waste management process described shows that ETF can effectively treat multi-source leachate.

4 Given the cradle-to-grave control of multi-source leachate, the treatment capabilities of the ETF, and the

5 regulatory control over this process, the ETF effluent "is not capable of posing a substantial present or

6 potential threat to public health or the environment. .." [WAC 173-303-072(4)]. This is ensured based on

7 the following.

9 - Waste being disposed into the lined RCRA-compliant disposal trenches must meet federal and state LDRs. Disposal trenches operate under strict adherence to waste acceptance criteria, which is verified for concentration based LDRs by sampling and analysis. This ensures metals are stabilized/immobilized, inorganics are deactivated, and organics are destroyed before disposal.

- When leachate is generated from the lined RCRA-compliant disposal trench, the leachate is designated with the F039 listed waste number, and characterized per the requirements of the LERF/ETF waste analysis plan, the sampling and generator provisions outlined in WAC 173-303-200 (40 CFR 262.34), as well as Sections 2.2 and 2.2.3 of this document. This ensures that LERF/ETF personnel are involved early in the leachate management process, thus minimizing problems with accepting the waste.

- ETF provides flexible and robust wastewater treatment capabilities using best available technology/all known and available technologies for chemical constituents. This ensures that if constituents are reasonably expected to be present in the leachate, these constituents will be destroyed or removed effectively from ETF treated effluent.

- Before treated effluent from ETF is land disposed, verification (by means of sampling and analysis) is performed to ensure the treated effluent meets all discharge requirements. If the effluent fails any of the strict controls, the effluent is rejected and retreated through ETF treatment units. 
This page intentionally left blank. 
DOE/RL-98-09, 1998 Report on Hanford Site Land Disposal Restrictions for Mixed Waste, U.S. Department of Energy, Richland Operations Office, Richland, Washington.

DOE/RL-97-03, Hanford Facility Dangerous Waste Permit Application, Liquid Effluent Retention Facility and 200 Area Effluent Treatment Facility, U.S. Department of Energy, Richland Operations Office, Richland, Washington.

DOE/RL-92-72, 200 Area Effluent Treatment Facility Delisting Petition, U.S. Department of Energy, Richland Operations Office, Richland, Washington.

DOE/RL-91-17, Hanford Facility Dangerous Waste Permit Application, Central Waste Complex, U.S. Department of Energy, Richland Operations Office, Richland, Washington.

DOE/RL-88-20, Hanford Facility Dangerous Waste Permit Application, Low-Level Burial Grounds, U.S. Department of Energy, Richland Operations Office, Richland, Washington.

DOE/RL-88-21, Hanford Facility Dangerous Waste Part A Permit Application, U.S. Department of Energy, Richland Operations Office, Richland, Washington.

DOE/RL-98-47, Environmental Restoration Disposal Facility Leachate Delisting Petition, Draft B, U.S. Department of Energy, Richland Operations Office, Richland, Washington.

Ecology, 1994, Dangerous Waste Portion of the Resource Conservation and Recovery Act Permit, Number WA7890008967, revised periodically, Washington State Department of Ecology, Olympia, Washington.

EPA, 1991, "A Project Manager's Guide to Requesting and Evaluating Chemical Analysis", EPA Contract No. $68 \mathrm{D} 80085$.

EPA 530/R-93/007, Petition to Delist Hazardous Wastes, A Guidance Manual, Second Edition, Prepared by the Science Applications International Corporation, Falls Church, VA for the Delisting Section Office of Solid Waste, U.S. Environmental Protection Agency, Washington, D.C.

EPA, 1994, Docket Report on Health-Based Levels and Solubilities Used in the Evaluation of Delisting Petitions, Submitted Under 40 CFR 260.20 and 260.22, Delisting Section, Waste Identification Branch, Office of Solid Waste, Environmental Protection Agency, Washington, D.C.

Hanford Facility RCRA Permit, Dangerous Waste Portion: Part III, Chapter 4, Liquid Effluent Retention Facility and 200 Area Effluent Treatment Facility; Part VI, Chapter 2, 183-H Solar Evaporation Basins.

HSDB, 1987, "Hazardous Substance Data Bank", National Library of Medicine, Bethesda, Maryland (CD-ROM version), MICROMEDEX, Inc., Englewood, Colorado.

ST 4500, State Waste Discharge Permit, State of Washington Department of Ecology, Washington State Department of Ecology, Olympia, Washington. 
1 WHC-SD-WM-TI-714, High-Density Polyethylene Liner Chemical Compatibility for Radioactive Mixed 2 Waste Trenches, Westinghouse Hanford Company, Richland, Washington.

60 FR 6054, "Hazardous Waste Management System; Identifications and Listing of Hazardous Waste; Proposed Exclusion".

60 FR 31115, "Hazardous Waste Management System; Identification and Listing of Hazardous Waste; Final Exclusion". 


\section{APPENDIX A}

EFFLUENT TREATMENT FACILITY ACTUAL TREATMENT EFFICIENCIES 
DOE/RL-98-62, Rev. 0

11/98

This page intentionally left blank. 


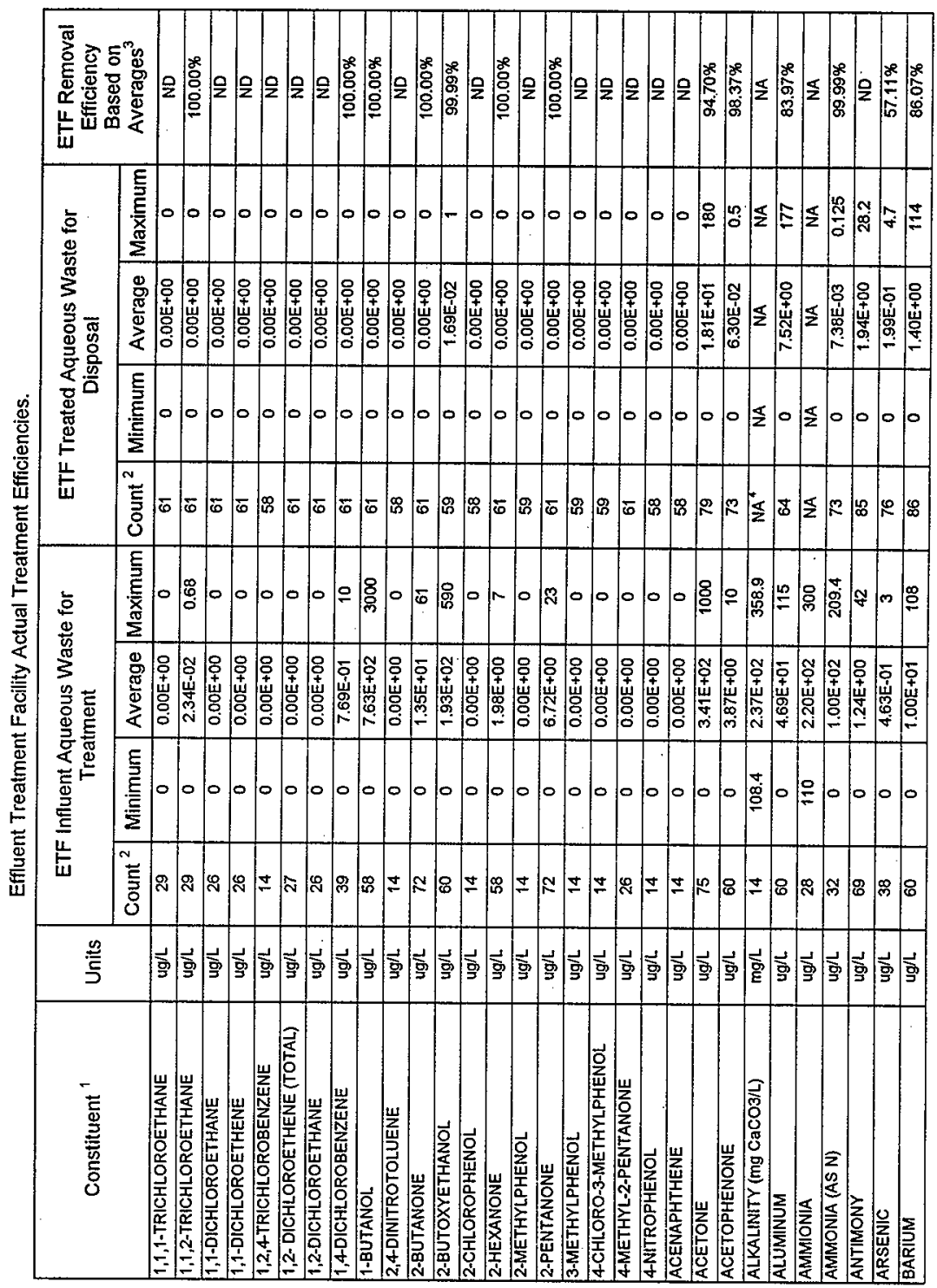


Effluent Treatment Facility Actual Treatment Efficiencies.

\begin{tabular}{|c|c|c|c|c|c|c|c|c|c|c|}
\hline \multirow[t]{2}{*}{ Constituent ${ }^{\prime}$} & \multirow[t]{2}{*}{ Units } & \multicolumn{4}{|c|}{$\begin{array}{c}\text { ETF Influent Aqueous Waste for } \\
\text { Treatment }\end{array}$} & \multicolumn{4}{|c|}{$\begin{array}{l}\text { ETF Treated Aqueous Waste for } \\
\text { Disposal } \\
\begin{array}{llll}\ldots & \ldots & \ldots & \end{array}\end{array}$} & \multirow{2}{*}{$\begin{array}{c}\text { ETF Removal } \\
\text { Efficiency } \\
\text { Based on } \\
\text { Averages }\end{array}$} \\
\hline & & Count $^{2}$ & Minimum & Average & Maximum & Count $^{2}$ & Minimum & Average & Maximum & \\
\hline BENZENE & $\mathrm{ug} / \mathrm{L}$ & 29 & 0 & $8.62 \bar{E}-02$ & 2.5 & 61 & $\overline{0}$ & $0.00 E+00$ & 0 & $100.00 \%$ \\
\hline BENZYL ALCOHOL & $\mathrm{ug} / \mathrm{L}$ & 44 & $\overline{0}$ & $2 . \overline{23 E+00}$ & 6 & 59 & 0 & $0.00 \mathrm{E}+00$ & 0 & $100.00 \%$ \\
\hline BERYLLIUM & $\mathrm{ug} / \mathrm{L}$ & 60 & 0 & $\overline{0.00 E+00}$ & 0 & 86 & 0 & $0.00 \mathrm{E}+00$ & 0 & ND \\
\hline BROMIDE & ug/L & 22 & 0 & $2.50 \mathrm{E}-02$ & 0.2 & 62 & $\overline{0}$ & $0.00 E+00$ & 0 & $100.00 \%$ \\
\hline BROMODICHLOROMETHANE & $\mathrm{ug} / \mathrm{L}$ & 3 & 0 & $0.00 E+00$ & 0 & $\overline{9}$ & 0 & $0.00 E+00$ & 0 & ND \\
\hline CADMIUM & $\mathrm{ug} / \mathrm{L}$ & 44 & 0 & $2.50 \mathrm{E}-02$ & 0.4 & 75 & 0 & $2.67 \mathrm{E}-03$ & 0.2 & ND \\
\hline CALCIUM & $\mathrm{ug} / \mathrm{t}$ & 60 & 259 & $1.11 \mathrm{E}+04$ & 112000 & 64 & 0 & $3.25 E+01$ & 327 & $99.71 \%$ \\
\hline CARBON DIȘULFIDE & $\mathrm{ug} / \mathrm{L}$ & 21 & 0 & $0.00 \mathrm{E}+00$ & 0 & 61 & 0 & $0.00 E+00$ & 0 & ND \\
\hline CARBON TETRACHLORIDE & $\mathrm{ug} / \mathrm{L}$ & 32 & 0 & $1.78 \mathrm{E}+01$ & 50 & 61 & 0 & $0.00 E+00$ & 0 & $100.00 \%$ \\
\hline CHLORIDE & $\mathrm{mg} / \mathrm{L}$ & 42 & 0 & $6.22 \mathrm{E}+00$ & 22.45 & 53 & 0 & $5.29 E-03$ & 0.15 & $99.92 \%$ \\
\hline CHLOROBENZENE & $\mathrm{ug} / \mathrm{L}$ & 29 & 0 & $0.00 E+00$ & 0 & 61 & 0 & $0.00 \mathrm{E}+00$ & 0 & ND \\
\hline CHLOROFORM & $\mathrm{ug} / \mathrm{L}$ & 32 & 0 & $1.89 E+00$ & 5.58 & 61 & 0 & 9.84E-03 & 0.6 & $99.48 \%$ \\
\hline CHROMIUM & $\mathrm{ug} / \mathrm{L}$ & 28 & 0 & $2.48 \mathrm{E}+00$ & 6.5 & 71 & 0 & 6.61E-01 & 13 & $73.35 \%$ \\
\hline COBALT & $\mathrm{ug} / \mathrm{L}$ & 60 & $\overline{0}$ & $1.26 \mathrm{E}-01$ & 7.54 & 64 & 0 & $3.18 \mathrm{E}-01$ & 11.6 & ND \\
\hline COPPER & $u g / L$ & 61 & 0 & $3.68 E+00$ & 11 & 91 & $\overline{0}$ & 5.35E-01 & 28 & $85.47 \%$ \\
\hline CYANIDE & $\operatorname{tg} / \mathrm{L}$ & 29 & 0 & $1.91 \mathrm{E}+00$ & 13.1 & 72 & 0 & 1.31E-01 & 4.64 & $93.14 \%$ \\
\hline DI-N-OCTYL. PHTHALATE & $\operatorname{tug} / \mathrm{L}$ & 14 & 0 & $7.86 \mathrm{E}-02$ & 1.1 & 59 & 0 & $0.00 \mathrm{E}+00$ & 0 & $100.00 \%$ \\
\hline FLUORIDE & $\mathrm{mg} / \mathrm{L}$ & 41 & 0 & $2.72 \mathrm{E}-01$ & 0.575 & 62 & 0 & 1:53E-02 & 0.1 & $94.37 \%$ \\
\hline FORMATE & $\mathrm{mg} / \mathrm{L}$ & 19 & 0 & $1.75 \mathrm{E}=01$ & 0.65 & NA & NA & NA & NA & NA \\
\hline HEXACHLOROETHANE & $\mathrm{ug} / \mathrm{L}$ & 14 & 0 & $0.00 E+00$ & 0 & 59 & 0 & $0.00 E+00$ & 0 & ND \\
\hline IRON & $\mathrm{ug} / \mathrm{L}$ & 60 & 0 & $2.52 \mathrm{E}+01$ & 161 & 64 & 0 & $1.39 \mathrm{E}+01$ & 115 & $44.73 \%$ \\
\hline LEAD & $4 \mathrm{gg} / \mathrm{L}$ & 40 & 0 & $1.50 \mathrm{E}-01$ & 2 & 75 & 0 & $8.25 E-02$ & 3 & $45.00 \%$ \\
\hline MAGNESIUM & ug/L & 60 & 0 & $3.57 \mathrm{E}+03$ & 36000 & 64 & 0 & $8.26 \mathrm{E}+00$ & 116 & $99.77 \%$ \\
\hline MANGANESE & $\mathrm{ug} / \mathrm{L}$ & 60 & 0 & 4.83E-01 & 8 & 64 & 0 & $1.73 E+00$ & 111 & ND \\
\hline MERCURY & ug/L & 61 & 0 & 8.03E-02 & 0.8 & 83 & 0 & $2.54 E-02$ & 0.6 & $68.41 \%$ \\
\hline METHYLENE CHLORIDE & $\mathrm{ug} / \mathrm{L}$ & 26 & 0 & $7.69 \mathrm{E}-02$ & 2 & 61 & 0 & $3.28 \mathrm{E}-02$ & 2 & ND \\
\hline N-NITROSODI-N.PROPYLAMINE & ug/L & 14 & 0 & $0.00 \mathrm{E}+00$ & 0 & 58 & 0 & $0.00 E+00$ & 0 & ND \\
\hline N-NITROSODIMETHYLAMINE & ug/L & 44 & 0 & $9.57 \mathrm{E}+00$ & 40 & 59 & 0 & $0.00 E+00$ & $\overline{0}$ & $100.00 \%$ \\
\hline NAPHTHALENE & ugg/L & 14 & 0 & $0.00 \mathrm{E}+00$ & 0 & 62 & $\overline{0}$ & $2.42 \mathrm{E}-02$ & 1 & NO \\
\hline NICKEL & $\mathrm{Ug} / \mathrm{L}$ & 60 & 0 & $2.88 \mathrm{E}+00$ & 48 & 86 & 0 & $1.65 E+00$ & 26.3 & $42.61 \%$ \\
\hline
\end{tabular}


Effluent Treatment Facility Actual Treatment Efficiencies.

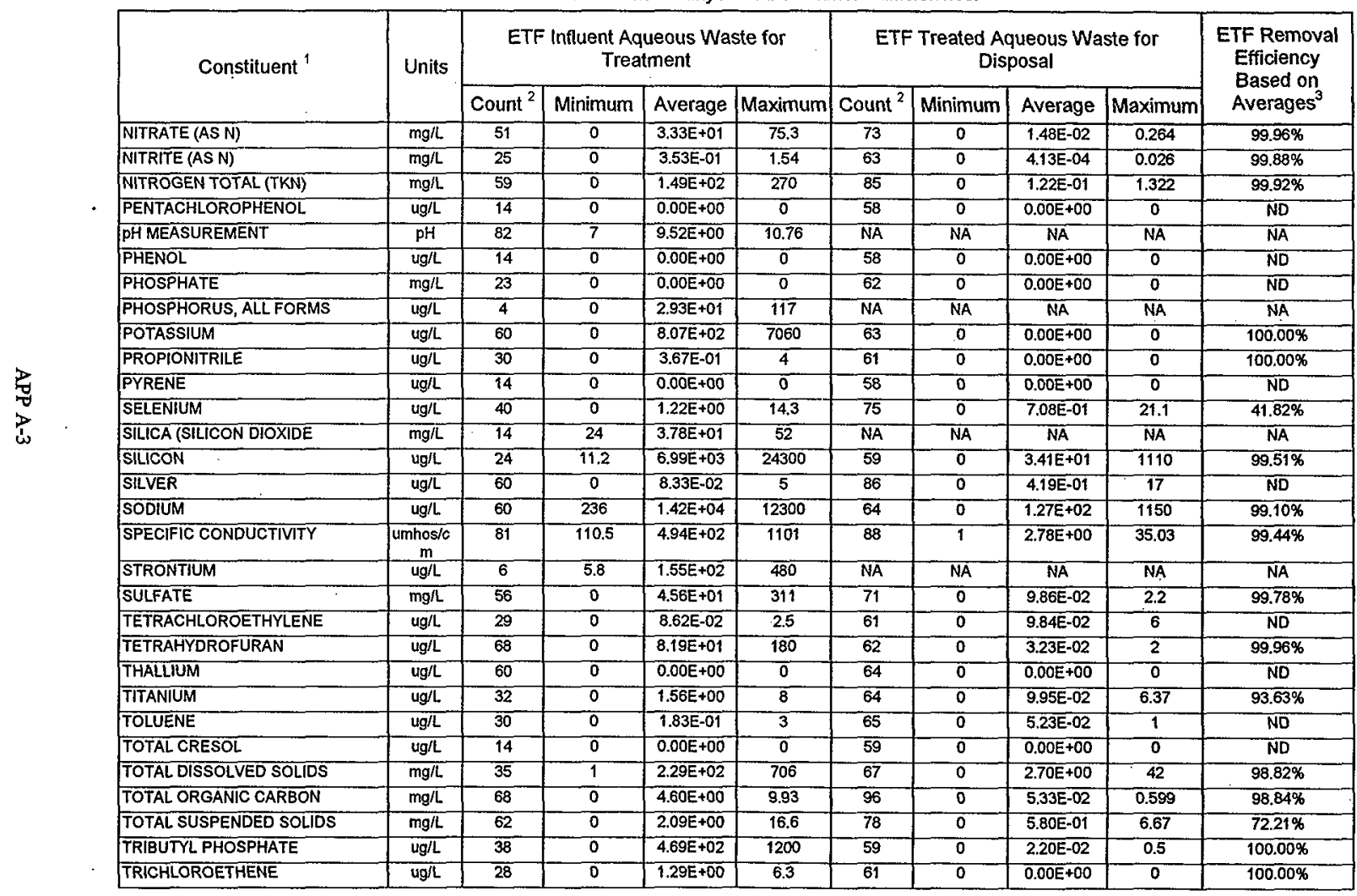




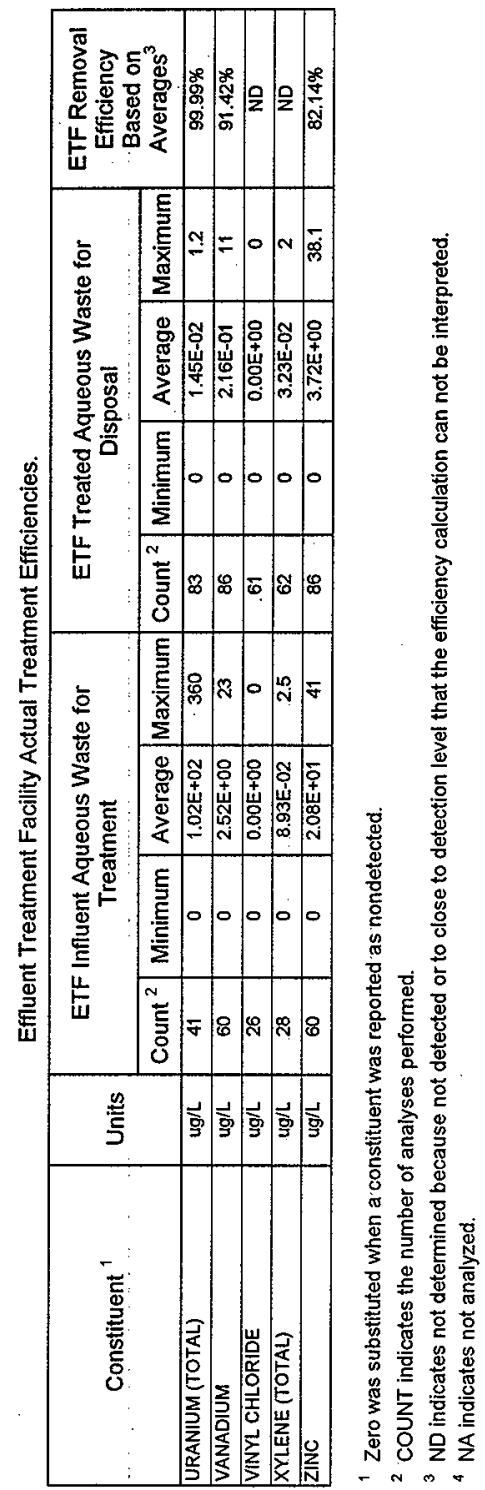




\section{APPENDIX B}

BACKGROUND INFORMATION USED TO DETERMINE THE TREATABUITY ENVELOPE FOR ORGANICS 
This page intentionally left blank.

APP B-ii 
Background Information Used to Determine the Treatability Envelope for Organics.

\begin{tabular}{|c|c|c|c|c|c|c|c|c|c|c|c|}
\hline $\begin{array}{c}\text { Treatability } \\
\text { Group }\end{array}$ & Constituent Name & CAS no. & Waste Number & \begin{tabular}{|c|} 
Final \\
Delisting
\end{tabular} & $\begin{array}{c}\text { Pilot } \\
\text { Testing }\end{array}$ & $\overline{H B L}(\mathrm{mg} / \mathrm{L})$ & $\begin{array}{l}\text { HBL Value } \\
\text { used for TBD } \\
(\mathrm{mg} / \mathrm{L})^{(1)}\end{array}$ & $\begin{array}{l}\text { Solubility } \\
\text { (mg/L) }\end{array}$ & \begin{tabular}{c|} 
Solubility \\
Preference
\end{tabular} & \begin{tabular}{|c|} 
LERF Liner \\
Compatibility \\
Limits (mg/L)
\end{tabular} & EE/O \\
\hline 1 & 2,4-Dimethylphenol & $105-67-9$ & U101 & & & 0.7 & & $5.90 \mathrm{E}+02$ & EPA & 2000 & 10 \\
\hline 1 & Coal tar creosote & $8007-45-2$ & & & & TBD & 1 & slightly soluble & HSDB & 100000 & 10 \\
\hline 1 & Creosote & $8001-58-9$ & & & & TBD & 1 & slightly soluble & HSDB & 100000 & 10 \\
\hline 1 & Cresol [Cresylic acid] & $1319-77-3$ & F001-5, U052 & c & $t$ & 2 & & $3.10 E+04$ & $\overline{E P A}$ & 100000 & 10 \\
\hline 1 & m-Cresol [3-Methylphenol] & $108-39-4$ & F001-5, U052 & & & TBD & 1 & $2.50 \mathrm{E}+04$ & HSDB & 100000 & 10 \\
\hline 1 & o.Cresol [2-Methylphenol] & $95-48-7$ & F001-5, U052 & & & TBD & 1 & $2.50 \mathrm{E}+04$ & HSOB & 100000 & 10 \\
\hline 1 & p-Cresol [4-Methylphenol] & 106-44-5 & F001-5, U052 & & & TBD & 1 & $2.50 E+04$ & HSDB & 100000 & 10 \\
\hline 1 & Phenol & 108-95-2 & U188 & & $t$ & 20 & & $9.30 E+04$ & $\overline{E P A}$ & 100000 & 4 \\
\hline 2 & $2,3,4,6$-Tetrachlorophenol & $58-90-2$ & $F 020-23, F 026-28$ & & & 1 & & $1.00 \mathrm{E}+03$ & EPA & 100000 & $\overline{10}$ \\
\hline 2 & $\begin{array}{l}2,3,4,6 \text {-tetrachlorophenol, potassitum salt } \\
{[2,3,4,6 \text {-tetrachlorophenol salt] }}\end{array}$ & $53535-27-6$ & & & & TBD & 1 & $1.00 E+03$ & EPA & 100000 & 10 \\
\hline 2 & $\begin{array}{l}2,3,4,6 \text {-tetrachlorophenol, sodium salt } \\
{[2,3,4,6 \text {-tetrachlorophenol, salt] }}\end{array}$ & $25567-55-9$ & & & & TBD & 1 & $1.00 \mathrm{E}+03$ & EPA & 100000 & 10 \\
\hline 2 & $2,4,5$-Trichiorophenol & $95-95-4$ & F020-23, F026-28 & & & 4 & & $1.19 \mathrm{E}+03$ & $\overline{E P A}$ & 100000 & 10 \\
\hline 2 & 2,4,6-Tribromophenol & 118.79-6 & & & & TBD & 1 & $7.00 \mathrm{E}+01$ & HSDB & 100000 & 10 \\
\hline 2 & 2,4,6-Trichlorophenol & 88-06-2 & $\mathrm{F} 020-23, \mathrm{~F} 026-28$ & & & 0.008 & & $8.00 E+02$ & EPA & 100000 & 10 \\
\hline 2 & 2,4-Dichlorophenol & 120-83-2 & U081 & & & 0.1 & & $4,60 E+03$ & EPA & 2000 & 10 \\
\hline 2 & 2,6-Dichlorophenol & $87-65-0$ & Uo82 & & & TBD & 1 & $4.60 \mathrm{E}+0 \overline{3}$ & HSOB & 2000 & 10 \\
\hline 2 & m-Chioro-3-methylphenol & 59-50-7 & 0039 & & & TBD & 1 & $\overline{3.85 E+03}$ & HSOB & 2000 & 10 \\
\hline 2 & o-Chlorophenol & $95-57-8$ & U048 & & & $\overline{0.2}$ & & $2.85 \mathrm{E}+04$ & $\overline{E P A}$ & 2000 & 10 \\
\hline 2 & Pentachlorophenol & $87-86-5$ & F020-23, F026-28 & & $\bar{t}$ & 0.001 & & $1.40 E+01$ & $\overline{E P A}$ & 100000 & 4 \\
\hline 2 & $\begin{array}{l}\text { Potasslum pentachlorophenate } \\
\text { [Pentachlorophenol salt] }\end{array}$ & $7778-73-6$ & & & & 0.001 & & $3.30 \mathrm{E}+05$ & HSDB & 100000 & 4 \\
\hline 2 & $\begin{array}{l}\text { Sodium pentachlorophenate } \\
\text { [Pentachlorophenol salt] }\end{array}$ & 131-52-2 & & & & 0.001 & & $3.30 \mathrm{E}+05$ & HSDB $\bar{B}$ & 100000 & $\overline{4}$ \\
\hline 3 & Acenaphthene & $83-32-9$ & F039 & & & 2 & & $3.42 \mathrm{E}+00$ & $\overline{E P A}$ & 2000 & 10 \\
\hline 3 & Acenaphthylene & 208-96-8 & F039 & & & TBD & 1 & $3.93 \mathrm{E}+00$ & HSDB & 2000 & 10 \\
\hline 3 & Anthracene & 120-12-7 & F039 & & & 10 & & $1.29 \mathrm{E}+00$ & EPA & 2000 & 10 \\
\hline 3 & Benzene & $71-43-2$ & F001-5, 0019 & c & $t$ & 0.005 & & $1.75 \mathrm{E}+03$ & EPA & 2000 & 3 \\
\hline 3 & Benzenearsonic acid (Arsenic) & 98-05-5 & & & & TBD & 1 & $2.50 E+04$ & MSOS & 100000 & 10 \\
\hline 3 & Fluorene & $86-73-7$ & F039 & & & 1 & & $1.69 \mathrm{E}+00$ & EPA & 2000 & 10 \\
\hline 3 & Naphthalene & $91-20-3$ & U051, U165 & $c$ & $t$ & 1 & & $3.40 \mathrm{E}+01$ & $\overline{E P A}$ & 2000 & 3 \\
\hline 3 & Fhenanthrene & $85-01-8$ & v051 & & & TBD & 1 & $1.60 E+0 \overline{0}$ & HSDB & 2000 & 10 \\
\hline 3 & Styrene & 100-42-5 & & & & 0.1 & & $3.00 E+02$ & $\overline{E P A}$ & 5000 & 7 \\
\hline 4 & 3-Methyicholanthrene & $56-49-5$ & U157 & & & $\begin{array}{c}0.0000000 \\
3 \\
\end{array}$ & & unk & & 2000 & 10 \\
\hline 4 & Benzo(a)anthracene & $56-55-3$ & प018 & & & 0.000004 & & $5.70 E-03$ & EPÁ & 2000 & 10. \\
\hline
\end{tabular}


Background Information Used to Determine the Treatability Envelope for Organics.

\begin{tabular}{|c|c|c|c|c|c|c|c|c|c|c|c|c|}
\hline $\begin{array}{c}\text { Treatability } \\
\text { Group }\end{array}$ & Constifuent Name & CAS no: & Waste Number & \begin{tabular}{|c|} 
Final \\
Delisting
\end{tabular} & $\begin{array}{c}\text { Pilot } \\
\text { Testing }\end{array}$ & $\mathrm{HBL}$ (mg/L) & $\begin{array}{c}\text { HBL Value } \\
\text { used for TBD } \\
\text { (mg/t) }\end{array}$ & $\begin{array}{c}\text { Solubility } \\
\text { (mg/L) }\end{array}$ & \begin{tabular}{|l|} 
Solubility \\
Preference
\end{tabular} & \begin{tabular}{c|} 
LERF Liner \\
Compatibility \\
Limits $(\mathrm{mg} / \mathrm{L})^{(2)}$
\end{tabular} & EE/O & \\
\hline 4 & Benzo(a)pyrene & $50-32-8$ & U022 & & & 0.0002 & & $1.20 \mathrm{E}-03$ & EPA: & 2000 & 10 & \\
\hline 4 & Benzo(b)fuoranthene & $205-99-2$ & $\bar{F} \overline{039}$ & & $\ldots .$. & 0.0001 & & $1.40 \mathrm{E}-02$ & EPA & 2000 & 10 & \\
\hline 4 & $\overline{B e n z o(g h i) p e r y l e n e}$ & $191-24-2$ & F039 & & & TBD & 1 & $2.60 \mathrm{E}-04$ & HSDB & 2000 & 10 & \\
\hline 4 & Benzo(k) fiuoranthene & $207-08-9$ & $\overline{F 039}$ & & & TBD & 1 & $7.60 \mathrm{E}-04$ & HSDB & 2000 & 10 & \\
\hline 4 & Chrysene & $218-01-9$ & 0050 & & & 0.001 & & $1.80 \mathrm{E}-03$ & EPA & 2000 & 10 & \\
\hline 4 & Dibenzo[a,e]pyrene & $192-65-4$ & F039 & & & TBD & 1 & unk & & 2000 & 10 & \\
\hline 4 & Dibenzola, h]anthracene & $53-70-3$ & 0063 & & & 0.000002 & & $5.00 E-04$ & EPA & 2000 & 10 & \\
\hline 4 & Fluoranthrene & $206-440$ & U120 & & & 1 & & $2.06 \mathrm{E}-01$ & EPA & 2000 & 10 & \\
\hline 4 & Indeno( $(1,2,3, \mathrm{~cd})$ pyrene & 193-39-5 & F039 & & & 0.0001 & & $5.30 \mathrm{E}-04$ & EPA & 2000 & 15 & \\
\hline 4 & Pyrene & 129-00-0 & 0051 & & $t$ & 1 & & $1.32 \mathrm{E}-01$ & EPA & 2000 & 4 & \\
\hline $5 a$ & $1,2,4,5$-Tetrachlorobenzene & $95-94-3$ & U207 & & & 0.01 & & $6.00 E+00$ & EPA & 2000 & 20 & \\
\hline$5 \longdiv { a }$ & 1,2,4-Trichlorobenzene & $120-82-1$ & F039 & & & 0.07 & & $3.00 E+01$ & EPA & 2000 & 15 & \\
\hline $5 \sqrt{a}$ & m-Dichlorobenzene & 541-73-1 & F001-5, U071 & & & TBD & 1 & $1.23 E+02$ & HSDB & 2000 & 15 & \\
\hline $5 / a$ & o-Dichlorobenzene & $95-50-1$ & 4070 & & & 0.6 & & $1.00 E+02$ & EPA & 2000 & 15 & \\
\hline $5 \mid a$ & p-Dichlorobenzene & $106-46-7$ & U072 & c & $t$ & 0.075 & & $7.90 E+01$ & EPA & 2000 & 5 & \\
\hline 5 & Pentachlorobenzene & 608-93-5 & U.183 & & & 0.03 & & 1.35E-01 & EPA & 2000 & 20 & \\
\hline 5 & Heptachlorodibenzofuran & $38998-75-3$ & & & & TBD & 1 & unk & & 2000 & 15 & \\
\hline 5 & Heptachlorodibenzo-p-dioxins & $35822-46-9$ & & & & TED & 1 & insoluble & CAMSOFT & 2000 & 15 & \\
\hline $6 \mid$ & Hexachloroethane & $67-72-1$ & U131 & c & $t$ & 0.006 & & $5.00 E+01$ & EPA & 2000 & 100 & \\
\hline $6 / \mathrm{b}$ & 1,3-Dichloropropene & $542-75-6$ & 0084 & & & 0.0005 & & $2.80 E+03$ & EPA & 2000 & 7 & \\
\hline $6 \mid \mathrm{b}$ & Chloroprene [2-Chloro-1,3-butadiene] & $126-99-8$ & F039 & & & 0.7 & & $3.00 \mathrm{E}+02$ & EPA & 2000 & 10 & \\
\hline $6 \sqrt{b}$ & cis-1,3-Dichloropropene & 10061-01-5 & 0084 & & & TBD & $\overline{1}$ & $2.70 \mathrm{E}+03$ & HSDB & 2000 & 7 & \\
\hline $6 \sqrt[b]{b}$ & Hexachlorobutadiene & $87-68-3$ & U128 & & & 0.001 & & $1.50 \mathrm{E}-01$ & EPA & 2000 & 10 & \\
\hline 6 & Hexachloropropene & 1888.71 .7 & U243 & & & 0.01 & & $4.00 \mathrm{E}-03$ & EPA & 2000 & 10 & \\
\hline \begin{tabular}{l|l}
7 & $\mathbf{a}$
\end{tabular} & bis(2-Chloroethoxy) methane & $111-91-1$ & U024 & & & $T B D$ & 1 & $8.10 E+04$ & HSDB & 2000 & 15 & 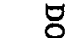 \\
\hline $7 \sqrt[a]{ }$ & Bis(2-Chloroethyl) ether & $111-44-4$ & U025 & & $t$ & 0.00008 & & $1.02 E+04$ & EPA & 2000 & 5 & 过 \\
\hline $7 \mid a$ & Bis(2-Chloroisopropyl) ether & $39638-32-9$ & U027 & & & 0.001 & & $1.70 \mathrm{E}+03$ & EPA & 2000 & 15 & \\
\hline $7 \bar{a}$ & Dichloroisopropyl ether & 108-60-1 & U027 & & & 0.001 & & $1.70 \mathrm{E}+03$ & EPA & 2000 & 15 & 6 \\
\hline $7 \bar{b}$ & 4-Bromophenylphenyi ether & 101-55-3 & U030 & & & TBD & 1 & insoluble & HSDB & 2000 & 10 & a \\
\hline $7 \mathbf{b}$ & 4-Chlorophenyl phenyl ether & $7005-72-3$ & & & $t$ & TBD & 1 & $3.30 \mathrm{E}+00$ & HSOB & 2000 & 4 & \\
\hline 8 & Bis(2-Ethylhexyl) phthalate & 117-81-7 & U028 & & $t$ & 0.006 & & $4.00 \mathrm{E}-01$ & EPA & 2000 & 5 & 告 \\
\hline 8 & Butylbenzyiphthalate & 85-68-7 & F039 & & & 0.1 & & $2.90 E+00$ & EPA & 200000 & 15 & $b_{\infty}^{4}$ \\
\hline
\end{tabular}


Background Information Used to Determine the Treatability Envelope for Organics.

\begin{tabular}{|c|c|c|c|c|c|c|c|c|c|c|c|}
\hline $\begin{array}{c}\text { Treatability } \\
\text { Group }\end{array}$ & Constituent Name & CASno. & Waste Number & \begin{tabular}{|c|} 
Final \\
Delisting
\end{tabular} & $\begin{array}{l}\text { Pilot } \\
\text { Testing }\end{array}$ & HBL (mg/L) & $\begin{array}{c}\text { HBL Value } \\
\text { used for TBD } \\
(\mathrm{mg} / \mathrm{L})^{(1)}\end{array}$ & $\begin{array}{c}\text { Solubility } \\
\text { (mg/L) }\end{array}$ & $\begin{array}{c}\text { Solubility } \\
\text { Preference }\end{array}$ & \begin{tabular}{|c|} 
LERF Liner \\
Compatibility \\
Limits (mg/L)
\end{tabular} & $E E / O$ \\
\hline 8 & Diethylphthalate & $84-66-2$ & U088 & & & 30 & & $8.96 \mathrm{E}+02$ & EPA & 100000 & 15 \\
\hline 8 & Dimethyl phthalate & $131-11-3$ & U102 & & & 400 & & $4.30 \mathrm{E}+\overline{0} \overline{3}$ & $\overline{E P A}$ & 100000 & 15 \\
\hline 8 & Di-n-butylphthalate & $84-74-2$ & 0069 & & & $\overline{4}$ & & $1.30 E+01$ & EPA & 100000 & 15 \\
\hline 8 & Di-n-octylphthalate & $117-84-0$ & U107 & $c$ & & 0.7 & & $3.00 \mathrm{E}+00$ & EPĀ & 100000 & 15 \\
\hline $9 \sqrt[a]{a}$ & 1-Butanol & $71-36-3$ & F001-5, U031 & $\mathrm{c}$ & $\mathrm{t}$ & 4 & & $9.10 \mathrm{E}+04$ & HSDB & 500000 & 10 \\
\hline $9 \mid \mathbf{a}$ & Benzyl alcohol & $100-51-6$ & & c & $t$ & TBD & 1 & $4.00 E+04$ & HSDB & 500000 & 10 \\
\hline $\begin{array}{r}\mathbf{a} \\
\end{array}$ & Isobutyl alcohol & $78-83-1$ & F001-5, U140 & & & 10 & & $7.60 E+04$ & EPA & 500000 & 20 \\
\hline $9 \sqrt[a]{a}$ & Methanol. & $67-56-1$ & F001-5, U154 & & & 20 & & $1.00 \mathrm{E}+06$ & EPA & 500000 & 15 \\
\hline 9 & 1,40ioxane [1,4-Diethyleneoxide] & $123-91-1$ & U108 & & & 0.008 & & $4.31 E+05$ & EPA & 2000 & 5 \\
\hline 9 & $2,4,5-T$ & $93-76-5$ & F039 & & & 0.4 & & $2.40 \mathrm{E}+02$ & EPA & 2000 & 20 \\
\hline 9 & $2,4,5$-TP [Silvex] & $93-72-1$ & [D017] & & & 0.05 & & $1.40 E+02$ & EPA & 2000 & 20 \\
\hline 9 & 2,4-D [2,4-Dichlorophenoxyacetic acid] & 94-75-7 & $\mathrm{U} 240$ & & & 0.07 & & $8.90 E+02$ & EPA & 2000 & 20 \\
\hline 9 & Carbofuran phenol & $1563-38-8$ & U367 & & & TBD & 1 & unk & & 2000 & 30 \\
\hline 9 & Ethyl Acetate & $141-78-6$ & F039 & & & 30 & & $1.00 \mathrm{E}+05$ & EPA & 100000 & 15 \\
\hline 9 & Kepone & $143-50-0$ & U142 & & & 0.000002 & & $7.60 E+00$ & EPA & 2000 & 15 \\
\hline 9 & Lead subacetate (Lead) & $1335-32-6$ & & & & TBD & 1 & $6.25 \mathrm{E}+04$ & HSDB & 100000 & 15 \\
\hline 9 & Methyl methacrylate & $80-62-6$ & U162 & & & 3 & & $2.00 E+01$ & EPA & 2000 & 20 \\
\hline 9 & Methyl methanesulfonate & $66-27-3$ & F039 & & & TBD & 1 & $2.00 E+05$ & HSDB & 2000 & 20 \\
\hline 9 & Phenyl mercuric acetate & $62-38-4$ & P092 & & & TBD & 1 & $4.37 \mathrm{E}+0.03$ & HSOB & 100000 & 15 \\
\hline 9 & Phthalic anhydride & $85-44-9$ & U190 & & & TBD & 1 & $6.17 E+03$ & HSDB & 2000 & 15 \\
\hline 9 & Safrole & $94-59-7$ & U203 & & & 0.0005 & & $1.50 \mathrm{E}+03$ & EPA & 2000 & 20 \\
\hline 9 & TCDD & 1746-01-6 & & & & TBD & 1 & $2.00 \mathrm{E}-05$ & HSOB & 2000 & $1 \overline{5}$ \\
\hline 9 & Vinyl acetate & 108-05-4 & & & & 40 & & $2.00 E+04$ & EPA & 100000 & 15 \\
\hline $10 a$ & 2-Acethylaminofluorene & 53-96-3 & U005 & & & TBD & 1 & $1.44 \mathrm{E}+02$ & HSDB & 2000 & 10 \\
\hline $10 a$ & 4Aminobiphenyi & $92-67-1$ & F039 & & & TBD & 1 & $8.42 \mathrm{E}+02$ & HSOB & 2000 & 10 \\
\hline$1 0 \longdiv { a }$ & Diphenylamine & $122-39-4$ & F039 & & & 0.9 & & $5.76 \mathrm{E}+01$ & EPA & 2000 & 15 \\
\hline $10 \mathbf{a}$ & o-Phenylenedlamine & 95-54-5 & & & & TBD & 1 & slightly soluble & HSDB & 2000 & 30 \\
\hline $10 a$ & Phenacetin [p-Acetophenetidide] & $62-44-2$ & U187 & & & TBD & 1 & $5.30 \mathrm{E}+02$ & HSOB & 2000 & 20 \\
\hline $10 a$ & p-Phenylenediamine & $106-50 \cdot 3$ & & & & 7 & & $3.80 \mathrm{E}+04$ & EPA & 2000 & 30 \\
\hline $10 \sqrt{a}$ & Pronamide & $23950-58-5$ & U192 & & & 0.005 & & $1.00 E+02$ & EPA & 2000 & 20 \\
\hline $10 a$ & Selenourea (Selenium) & $630-10-4$ & & & & TBD & 1 & soluble & HSDB & 2000 & 30 \\
\hline $10 \mathrm{a}$ & Toluene-2,4-diamine & $95-80-7$ & & & & 0.00003 & & $4.77 \mathrm{E}+04$ & EPA & 2000 & 30 \\
\hline $10 \sqrt[b]{ }$ & 4,4-Methylenebis(2-chloroaniline) & $101-14-4$ & U158 & & & TBD & 1 & Insoluble & HSDB & 2000 & 10 \\
\hline $10 \mathrm{~b}$ & Aniline & 62-53-3 & U012 & & $t$ & 0.01 & & $3.50 E+04$ & EPA & 2000 & 4 \\
\hline 10$]$ & o-Nitroanillne & $88-74-4$ & & & & TBD & 1 & $1.47 E+03$ & HSDB & 2000 & 10 \\
\hline
\end{tabular}


Background Information Used to Determine the Treatability Envelope for Organics.

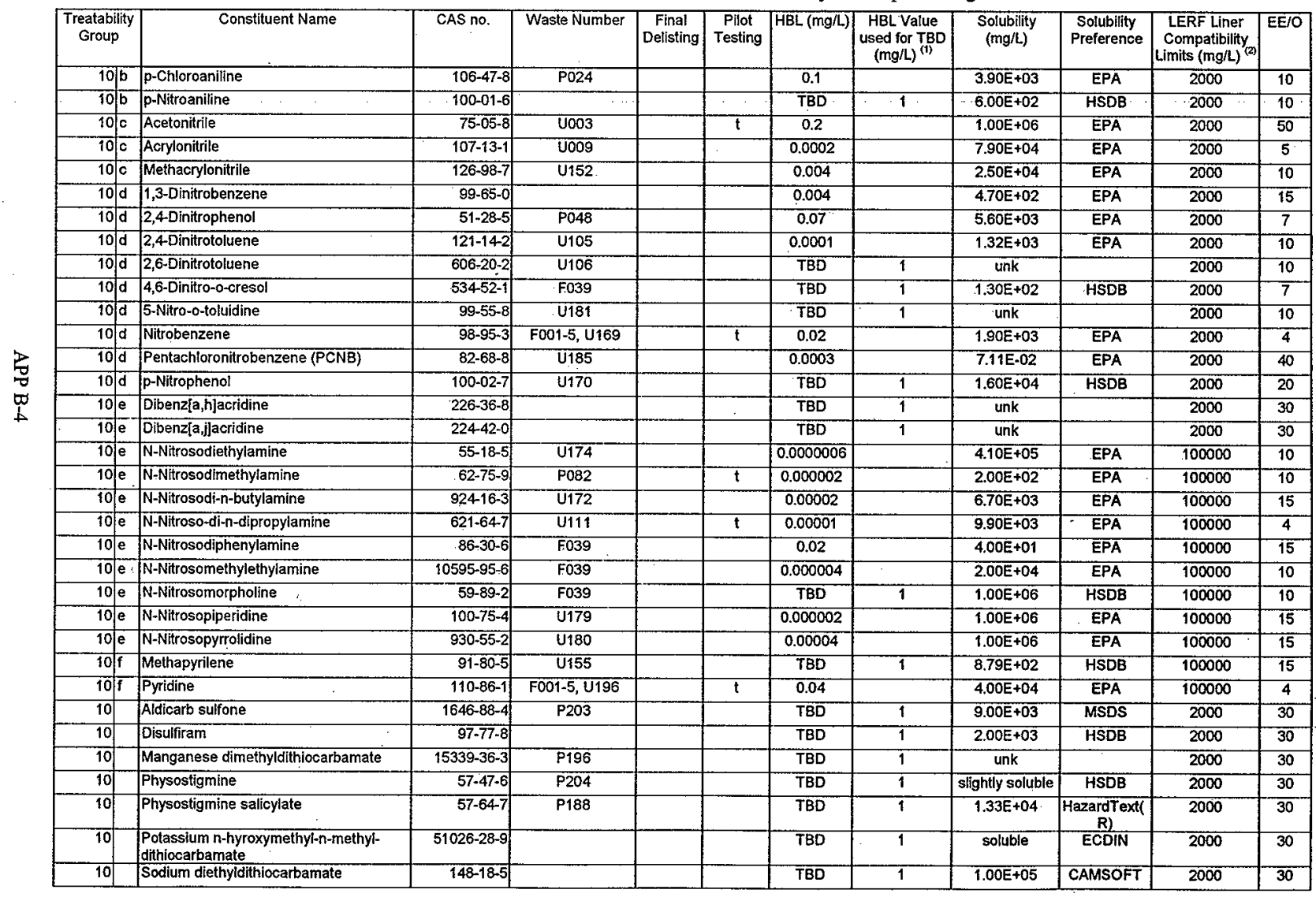


Background Information Used to Determine the Treatability Envelope for Organics.

\begin{tabular}{|c|c|c|c|c|c|c|c|c|c|c|c|}
\hline $\begin{array}{l}\text { Treatability } \\
\text { Group }\end{array}$ & Constituent Name & CAS no. & Waste Number & \begin{tabular}{|c|} 
Final \\
Delisting
\end{tabular} & $\begin{array}{c}\text { Pilot } \\
\text { Testing }\end{array}$ & $\mathrm{HBL}$ (mg/L) & \begin{tabular}{|c|} 
HBL Value \\
used for TBD \\
$(\mathrm{mg} / \mathrm{L})^{(1)}$
\end{tabular} & $\begin{array}{c}\text { Solubility } \\
\text { (mg/L) }\end{array}$ & $\begin{array}{c}\text { Solubility } \\
\text { Preference }\end{array}$ & \begin{tabular}{|c|} 
LERF Liner \\
Compatibility \\
Limits (mg/L) ${ }^{(2)}$
\end{tabular} & $E E / O$ \\
\hline 10 & Tetrabutylthiuram disulfide & $1634-02-2$ & & & & TBD & $\overline{1}$ & unk & & 2000 & 30 \\
\hline 11 & A2213 & $30558-43-1$ & U394 & & & TBD & 1 & unk & & 2000 & 30 \\
\hline 11 & Âldrin & $309-00-2$ & $\overline{P 004}$ & & & 0.000005 & & $1.80 \mathrm{E}-01$ & EPA & 2000 & 30 \\
\hline 11 & Alpha-BHC & 319-84-6 & U129 & & & 0.00001 & & $1.63 E+00$ & $\overline{E P A}$ & $20 \overline{0}$ & $\overline{60}$ \\
\hline 11 & Aramite & $140-57-8$ & F039 & & & 0.003 & T. & $1,00 \mathrm{E}-01$ & HSDB & 2000 & 30 \\
\hline 11 & Barban & 101-27-9 & U280 & & & TBD & 1 & $1.10 \mathrm{E}+01$ & HSDB & 2000 & 30 \\
\hline 11 & Bendiocarb & $22781-23-3$ & $\mathrm{U} 278$ & & & TBD & 1 & $4.00 \mathrm{E}+01$ & HSDB & 2000 & 30 \\
\hline 11 & Benomyl & $1780435-2$ & $\mathrm{U} 271$ & & & TBD & 1 & $3.80 \mathrm{E}+00$ & HSDB & 2000 & 30 \\
\hline 11 & Beta-BHC & 319-85-7 & U129 & & & 0.00005 & & $2,40 E-01$ & EPA & 2000 & 60 \\
\hline 11 & Chlordane & 57-74-9 & D020, U036 & & & 0.002 & & $5.6 \mathrm{E}-01$ & EPA & 2000 & 60 \\
\hline 11 & Butylate & $2008-41-5$ & & & & TBD & 1 & $4.40 E+01$ & HSDB & 2000 & 30 \\
\hline 11 & Carbary & $63-25-2$ & $\mathrm{U} 279$ & & & TBD & 1 & $1.20 E+02$ & HSDB & 2000 & 30 \\
\hline 11 & Carbendazim & $10605-21-7$ & U372 & & & TBD & 1 & insoluble & MSDS & 2000 & 30 \\
\hline 11 & Carbofuran & $1563-66-2$ & P127 & & & TBD & 1 & $7.00 \mathrm{E}+02$ & HSDB & 2000 & 30 \\
\hline 11 & Copper dimethyldithiocarbamate & $137-29-1$ & & & & TBD & 1 & unk & & 2000 & 30 \\
\hline 11 & DDD (p,p"-DDD) & 72-54-8 & U060, U061 & & & 0.0004 & & $1.00 \mathrm{E}-01$ & EPA & 2000 & 30 \\
\hline 11 & DDE $\left(p, p^{\prime}-D D E\right)$ & $72-55-9$ & U061 & & & 0.0003 & & $4.00 \mathrm{E}-02$ & EPA & 2000 & 30 \\
\hline 11 & DDT $\left(p, p^{-D D T}\right)$ & $50-29-3$ & U061 & & & 0.0003 & & 5.00E-03 & EPA & 2000 & 30 \\
\hline 11 & Delta-BHC & 319-86-8 & U129 & & & TBD & 1 & $1.00 E+01$ & HSDB & 2000 & 60 \\
\hline 11 & Dieldrin & $60-57-1$ & P037 & & & 0.000005 & & $1,95 \mathrm{E}-01$ & EPA & 2000 & 30 \\
\hline 11 & Dinoseb & $88-85-7$ & P020 & & & 0.007 & & $5.00 \mathrm{E}+01$ & EPA & 2000 & 30 \\
\hline 11 & Disolfoton & $298-04-4$ & $\mathrm{P} 039$ & & & 0.001 & & $2.50 E+01$ & EPA & 2000 & 20 \\
\hline 11 & Endosulfan I & $959-98-8$ & P050 & & & TBD & 1 & unk & & 2000 & 20 \\
\hline 11 & Endosulfan II & $33213-65-9$ & $\overline{P 050}$ & & & TBD & 1 & unk & & 2000 & 20 \\
\hline 11 & Endosulfan suifate & $1031-07-8$ & P050 & & & TBD & 1 & 1.17E-01 & HSDB & 2000 & 20 \\
\hline 11 & Endrin & $72-20-8$ & P051 & & & 0.002 & & $2.50 \mathrm{E}-01$ & EPA & 2000 & $20^{\circ}$ \\
\hline 11 & Endrin aldehyde & $7421-93-4$ & P051 & & & TBD & 1 & $250 \mathrm{E}-01$ & HSDB & 2000 & 20 \\
\hline 11 & EPTC & $755-94-4$ & & & & TBD & 1 & $3.75 E+02$ & HSDB & 2000 & 30 \\
\hline 11 & Ethyi Ziram & $14324-55-1$ & & & & TBD & 1 & insoluble & HSDB & 2000 & 30 \\
\hline 11 & Famphur & 52-85-7 & P097 & & & $\overline{0.001}$ & & $1 . \overline{43 E+02}$ & EPA & 2000 & 20 \\
\hline 11 & Ferbam & 14484-64-1 & & & & TBD & 1 & $1.20 \mathrm{E}+02$ & HSDB & 2000 & 30 \\
\hline 11 & Heptachlor & $76-44-8$ & $\overline{P 059}$ & & & 0.0004 & & $1.80 \mathrm{E}-01$ & EPA & 2000 & 60 \\
\hline 11 & Heptachlor epoxide & $1024-57-3$ & $\overline{P 059}$ & & & 0.0002 & & $3 . \overline{00 E-01}$ & $\overline{E P A}$ & 2000 & 20 \\
\hline 11 & Isodrin & 465-73-6 & $\overline{P 060}$ & & & TBD & 1 & unk & & 2000 & 20 \\
\hline 11 & Lindane [gamma-BHC] & 58-89-9 & U129 & & $t$ & 0.0002 & & $7.80 E+00$ & $\overline{E P A}$ & 2000 & 40 \\
\hline
\end{tabular}


Background Information Used to Determine the Treatability Envelope for Organics.

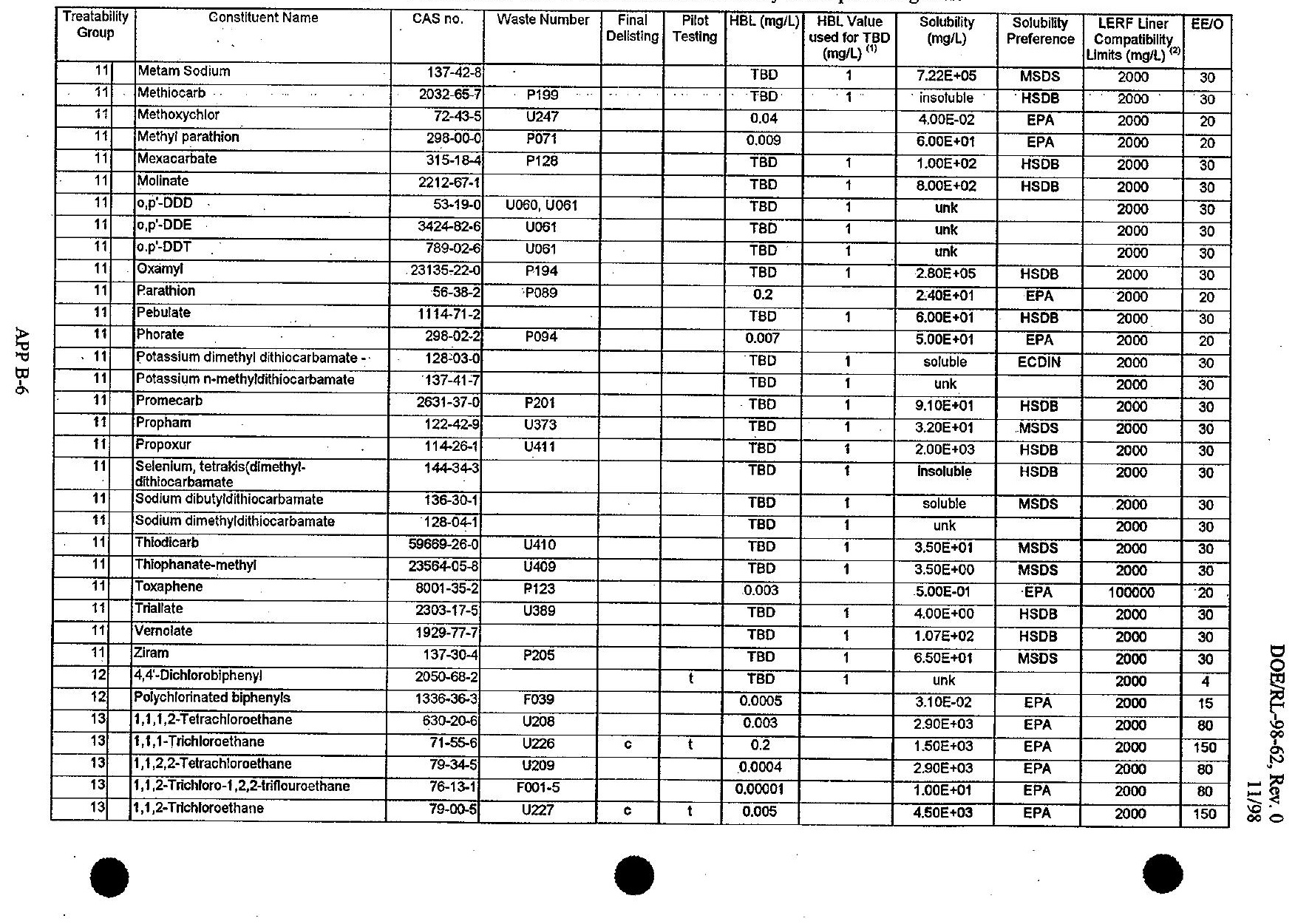


Background Information Used to Determine the Treatability Envelope for Organics.

\begin{tabular}{|c|c|c|c|c|c|c|c|c|c|c|c|}
\hline $\begin{array}{l}\text { Treatabllity } \\
\text { Group }\end{array}$ & Constituent Name & CASno. & Waste Number & \begin{tabular}{|c|} 
Final \\
Delisting
\end{tabular} & $\begin{array}{l}\text { Pilot } \\
\text { Testing }\end{array}$ & $\mathrm{HBL}(\mathrm{mg} / \mathrm{L})$ & $\begin{array}{c}\text { HBL Value } \\
\text { used for TBD } \\
\text { (mg/L) }^{(1)}\end{array}$ & $\begin{array}{c}\text { Solubility } \\
\text { (mg/L) }\end{array}$ & $\begin{array}{c}\text { Solubility } \\
\text { Preference }\end{array}$ & $\begin{array}{c}\text { LERF Liner } \\
\text { Compatibility } \\
\text { Limits }(\mathrm{mg} / \mathrm{L})^{(2)}\end{array}$ & EE/O \\
\hline 13 & 1,1-Dichloroethane & $75-34-3$ & U076 & & & 0.0009 & & $5.50 \mathrm{E}+03$ & $\overline{E P A}$ & 2000 & 40 \\
\hline 13 & 1,2,3-Trichloropropane & $96-18-4$ & F039 & & & 0.00001 & & $4.00 \mathrm{E}+03$ & EPA & 2000 & 40 \\
\hline 13 & 1,2, Dibromo-3-chloropropane & 96-12-8 & U066 & & & 0.0002 & & $1.00 E+03$ & EPA & 2000 & 40 \\
\hline 13 & $\begin{array}{l}\text { 1,2-Dichloropropane [Propylene } \\
\text { dichloridel }\end{array}$ & $78-87-5$ & U083 & & & 0.005 & & $2.70 \overline{E+03}$ & EPA & 2000 & 40 \\
\hline 13 & Bromodichloromethane & $75-27-4$ & F039 & & & 0.001 & & $4.70 \mathrm{E}+03$ & $\overline{E P A}$ & 2000 & 40 \\
\hline 13 & Bromoform & $75-25-2$ & $\mathrm{U} 225$ & & & 0.01 & & $3.01 \mathrm{E}+03$ & EPA & 2000 & 60 \\
\hline 13 & Bromomethane [Methyl bromide] & $7483-9$ & U029 & & & 0.05 & & $1.00 E+03$ & $\overline{E P A}$ & 2000 & 40 \\
\hline 13 & Carbon tetrachloride & $56-23-5$ & F001-5, U211 & c & $t$ & 0.005 & & $7.57 €+02$ & EPA & 2000 & 200 \\
\hline 13 & Chloroethane & 75-00-3 & F039 & & & TBD & 1 & $5 . \overline{4 E+03}$ & HSDB & 2000 & 40 \\
\hline 13 & Chloroform & $67-66-3$ & U044 & c & $t$ & 0.01 & & $8.20 \mathrm{E}+03$ & EPA & 2000 & 100 \\
\hline 13 & Chloromethane [Methyl chloride] & $7487-3$ & U045 & & & 0.007 & & $6.50 \mathrm{E}+03$ & EPÁ & 2000 & 30 \\
\hline 13 & Dibromochloromethane & 124.48-1 & F039 & & & 0.001 & & $4.40 \mathrm{E}+03$ & EPA & 2000 & 40 \\
\hline 13 & Dibromomethane [Methyiene bromide] & 74-95-3 & U068 & & & 0.4 & & $1.30 \overline{\mathrm{E}+04}$ & EPA & 2000 & 30 \\
\hline 13 & Dichlorodifiluoromethane & $75-71-8$ & U075 & & & 7 & & $2.80 \mathrm{E}+02$ & EPA & 2000 & 80 \\
\hline 13 & Ethylene dibromide [1,2-Dibromoethane] & 106-93-4 & U067 & & & 0.00005 & & $4.30 E+03$ & EPA & 2000 & 40 \\
\hline 13 & Ethylene dichloride [1,2-Dichloroethane] & 107-06-2 & U077 & c & & 0.005 & , & $8.52 \mathrm{E}+03$ & $\overline{E P A}$ & 2000 & 40 \\
\hline 13 & lodomethane [Methyl iodide] & 74-88-4 & U138 & & & TBD & 1 & $1.40 \mathrm{E}+04$ & HSDB & 2000 & 30 \\
\hline 13 & Methylene chtoride [Dichloromethane] & $75-09-2$ & U080 & & $t$ & 0.005 & & $2.00 E+04$ & EPA & 2000 & 60 \\
\hline 13 & Trichlorofluoromethane & $75-69-4$ & F001-5, U121 & & & 10 & & $1.10 \mathrm{E}+03$ & EPA & 2000 & 80 \\
\hline 14 & 1,1-Dichloroethylene & $75-35-4$ & U078 & $c$ & & 0.007 & 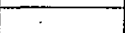 & $2.25 E+03$ & $\overline{E P A}$ & 2000 & 4 \\
\hline 14 & Allyl chloride [3-Chloropropene] & 107-05-1 & F039 & & & 0.004 & & $1.00 \mathrm{E}+02$ & EPÁ & 2000 & 5 \\
\hline 14 & cls-1,2-Dicloroethylene & 156-59-2 & & & & 0.07 & & $3.50 E+03$ & EPA & 2000 & 5 \\
\hline 14 & Tetrachloroethene [Tetrachloroethylene] & 127-18-4 & F001-5, U210 & $\bar{c}$ & $t$ & 0.005 & & $1.50 \mathrm{E}+02$ & EPA & 2000 & 4 \\
\hline 14 & trans-1,2-Dichloroethylene & $156-60-5$ & $\bar{U} 079$ & & & 0.1 & & $6.30 \bar{E}+03$ & EPA & 2000 & 5 \\
\hline 14 & trans-1,3-Dichloropropene & 10061-02-6 & U084 & & & TBD & 1 & $2.80 E+03$ & EPA & 2000 & 5 \\
\hline 14 & Trichloroethylene & $79-01-6$ & F001-5, U228 & c & & 0.005 & & $1.10 \mathrm{E}+03$ & EPA & 2000 & 4 \\
\hline 14 & Vinyl chloride & $75-01-4$ & U043 & c & & 0.002 & & $2.67 \overline{E+03}$ & EPA & 2000 & 4 \\
\hline 15 a & Ethylbenzene & 100-41-4 & F001-5 & & & 0.7 & & $1.52 \mathrm{E}+02$ & EPA & 2000 & 7 \\
\hline$1 5 \longdiv { \mathbf { a } }$ & Toluene & 108-88-3 & $\begin{array}{l}\text { F001-5, U051, } \\
\text { U220 }\end{array}$ & c & $t$ & 1 & & $5.35 \mathrm{E}+02$ & EPA & 2000 & 2 \\
\hline $15 \sqrt{a}$ & Xyienes (total) & $1330-20-7$ & U051, U239 & & & 10 & & $1.98 \mathrm{E}+02$ & $\overline{E P A}$ & 2000 & 7 \\
\hline 16 & Chlorobenzene & 108-90-7 & F001-5, U037 & $c$ & & 0.1 & & $4.66 \mathrm{E}+02$ & EPA & 2000 & 5 \\
\hline 16 & Dichlorophenylarsine (Arsenlc) & 696-28-6 & . & & & TBD & & insoluble & HSDE & 2000 & 4 \\
\hline \begin{tabular}{l|l|l|}
17 & $a$ \\
\end{tabular} & Acrolein & $107-02-8$ & $\mathrm{P} 003$ & & $t$ & 0.7 & & $5.00 \mathrm{E}+05$ & EPA & 2000 & 4 \\
\hline $18 \mathrm{a}$ & isosafrole & 120-58-1 & U141 & & & TBD & 1 & Insoluble & HSOB & 2000 & 15 \\
\hline
\end{tabular}




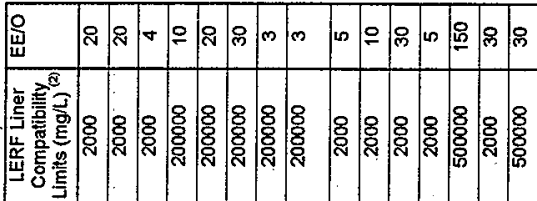

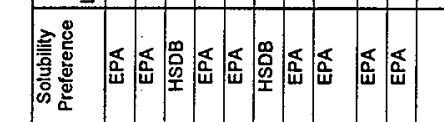

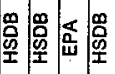

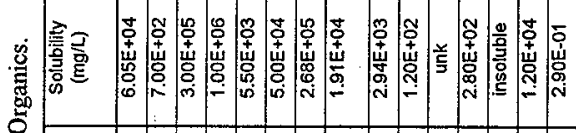

s

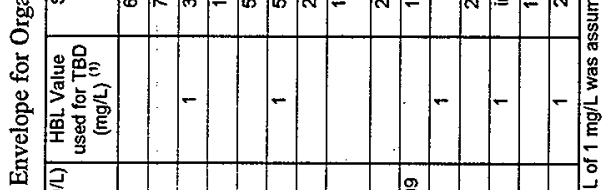

:

要

.

\begin{tabular}{lll|l|l|l|l|l} 
& 10 & 0 & 0 & & 0 & \\
\hline
\end{tabular}

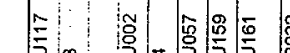

5100

wis.

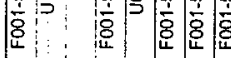

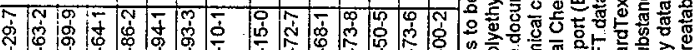

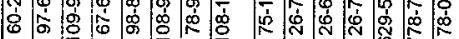

政

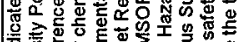

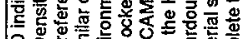

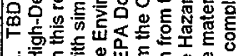

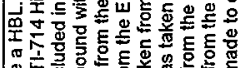

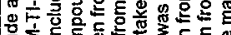

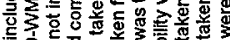

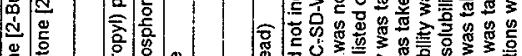

兽

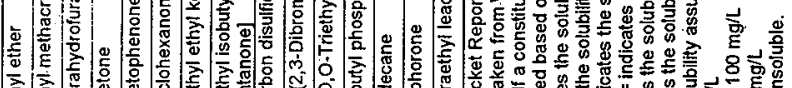

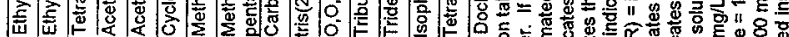

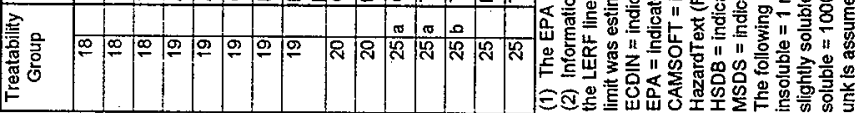




\section{DISTRIBUTION}

$\underline{\text { MSIN }}$

Washington State Department of Ecology

M.N. Jaraysi

B5-18

R. J. Julian

B5-18

S. J. Skurla

B5-18

M. A. Wilson

B5-18

U.S. Environmental Protection Agency

Dave Bartus-Region 10

D. R. Sherwood-Region 10

B5-01

J. Wilkinson

Confederated Tribes of the Umatilla Indian Nation

P. O. Box 638

Pendleton, Oregon 97801

D. Powaukee

Nez Perce Tribe

P. O. Box 365

Lapwai, Idaho 93540

R. Jim, Manager

Environmental Restoration/Waste Management Program Yakama Indian Nation

P. O. Box 151

Toppenish, Washington 98948

U.S. Department of Energy, Richland Operations Office

H. E. Bilson

S7-41

E. M. Bowers (2)

S7-55

C. E. Clark

A5-15

A. C. McKarns

A5-15

J. E. Rasmussen

A5-15

G. L. Sinton

S7-55

Reading Room

H2-53

Bechtel Hanford, Inc.

F. V. Roeck

H0-17

Fluor Daniel Hanford. Inc.

A. G. Miskho

H6-23

S. M. Price

H6-23

B. D. Williamson

B3-15

Pacific Northwest National Laboratory

Hanford Technical Library

P8-55 


\section{DISTRIBUTION}

Waste Management Federal Services of Hanford, Inc.

$\underline{\text { MSIN }}$

L. T. Blackford

T4-05

R. R. Bloom

S6-71

R. C. Bowman (2)

H6-24

R. H. Engelmann

H6-24

D. L. Flyckt

S6-71

T. L. Moore

H6-30

D. E. Nester

H6-06

P. M. Olson

R3-45

J. A. Winterhalder

H6-21

Permitting Documentation (5)

L4-97

Lockheed Martin Services, Inc.

Central Files

B1-07

DPC

H6-08

EDMC (2)

H6-08 